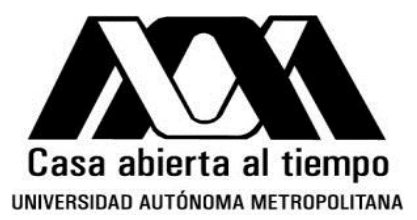

UNIVERSIDAD AUTÓNOMA METROPOLITANA - IZTAPALAPA DIVISIÓN DE CIENCIAS BÁSICAS E INGENERÍA

\title{
ESPACIOS DE BERGMAN PESADOS
}

\author{
Tesis que presenta \\ Luis Javier Carmona Lomeli \\ Para obtener el grado de \\ Doctor en Ciencias Matemáticas
}

Asesor:

Dr. Lino Feliciano Reséndis Ocampo

Jurado Calificador:

Presidente: Dr. Antoni Wawrzyñczyk Wilkiewicz

Secretario: Dr. Luis Verde Star

Vocal: $\quad$ Dra. María de Lourdes Palacios Fabila

Vocal: $\quad$ Dra. Maribel Loaiza Leyva

Vocal: $\quad$ Dr. Luis Manuel Tovar Sánchez

Ciudad de México

28 de Abril de 2017 


\title{
DEDICATORIAS
}

\section{A mi Padre y Madre:}

\author{
José y Eleuteria
}

Quienes me trajeron a este mundo y sin escatimar esfuerzo alguno sacrificaron gran parte de su vida para educarme. Jamás encontraré la forma de agradecer su apoyo, comprensión y confianza. Mis logros son también suyos e inspirados en ellos, hago de ellos éste triunfo y quiero compartirlo por siempre con ustedes.

\section{A mi Esposa e Hijas}

Rosario, Rosario Elizabeth y Esmeralda Sofia.

A mi esposa por su constante apoyo, comprensión, amor y por darme la gran alegría de ser pápa. A mis hijas por ser un nuevo motor en mi vida para seguir adelante.

\section{A mis Hermanos y Hermana:}

Juan Pablo, Sandra Elizabeth y José Emilio

Para quienes su ilusión ha sido verme convertido en un hombre de provecho. Y que siempre están a mi lado para alentarme a seguir. 


\section{AGRADECIMIENTOS}

\section{A mi Director de Tesis:}

Dr. Lino Feliciano Reséndis Ocampo

Por dirigirme en este trabajo de tesis, dedicándome su invaluable ayuda, tiempo y atención. Por ser una parte esencial de mi formación académica y sobre todo por la amistad y confianza brindada.

\section{A mis Revisores de Tesis:}

Dra. Maribel Loaiza Leyva, Dra. María de Lourdes Palacios Fabila, Dr. Antoni Wawrzynczyk Wilkiewicz, Dr. Luis Verde Star y Dr. Luis Manuel Tovar Sánchez.

Por el tiempo dedicado a la revisión de esta tesis, así como las sugerencias que me hicieron para hacer de ella un mejor trabajo.

\section{A la Maestra Iseo González:}

Por todo el apoyo brindado referente a los trámites administrativos dentro del posgrado. 


\section{Índice general}

Índice general V

Resumen

Introducción

1. Algunos operadores integrales en los espacios $L^{p} \quad 1$

1.1. Operadores acotados en espacios de Banach . . . . . . . . . . . . . . 1

1.2. Desigualdad de Hölder . . . . . . . . . . . . . . . . . . . . . . 2

1.3. Teorema de Schur . . . . . . . . . . . . . . . . . . 2

1.4. Operadores integrales en el disco unitario $\mathbb{D}$. . . . . . . . . . 3

1.5. Espacios de Bergman . . . . . . . . . . . . . . . . . 4

2. El Espacio $V M O(\mathbb{D}) \quad 11$

2.1. Transformaciones de Möbius . . . . . . . . . . . . . . . 11

2.2. El espacio de Bloch . . . . . . . . . . . . . . . . . . . . . . 13

2.3. La Transformada de Berezin . . . . . . . . . . . . . . . . . . 16

2.4. Estimaciones integrales tipo Littlewood-Paley . . . . . . . . . . . . 18

2.5. El espacio $\mathrm{VMO}$. . . . . . . . . . . . . . . . . . . 22

3. Espacios de Bergman Pesados ${ }_{s} \mathcal{A}_{q}^{p} \quad 27$

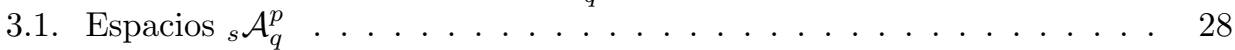

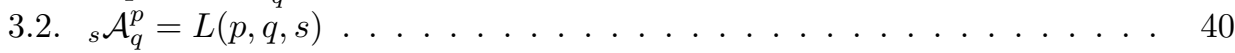

3.3. Inclusiones Estrictas . . . . . . . . . . . . . . . . . 43

4. El operador de Bergman $P_{\beta}$ en los espacios $L(p, q, s)={ }_{s} \mathcal{A}_{q}^{p} \quad \mathbf{5 1}$

4.1. Una Estimación Integral . . . . . . . . . . . . . . . . . . . . . . 51

4.2. El operador de Bergman $P_{\beta}$ en $L(p, q, s)={ }_{s} \mathcal{A}_{q}^{p} \ldots \ldots 6$

$\begin{array}{ll}\text { Conclusiones y Perspectivas } & 69\end{array}$ 
Apéndice

71

Bibliografía

75

Índice alfabético

77 


\section{Resumen}

Este trabajo es un estudio sobre uno de los espacios pesados de funciones analíticas más importantes: El espacio de Bergman pesado. Se estudian algunas de sus propiedades, caracterizaciones y la relación que tiene con otros espacios de funciones analitícas.

En el primer capítulo se enuncian definiciones y resultados conocidos sobre operadores lineales en espacios de Banach. Se enuncia la desigualdad de Hölder, la cual es una estimación fundamental en el estudio de integrales en general y en particular de operadores integrales. También se presenta el Lema de Schur el cual da condiciones para que cierto operador integral sea acotado en $L^{p}\left(\mathbb{D}, d A_{\alpha}\right)$ y se da una estimación integral (ver Teorema 1.4.2) la cual es fundamental en el estudio de operadores en espacios de funciones en el disco unitario. Por último se define el espacio de Bergman, la proyección de Bergman y a partir de ellos se estudia una familia de operadores integrales en el disco unitario cuyo núcleo está formado a partir del núcleo de Bergman.

En el segundo capítulo se enumeran propiedades generales de las transformaciones de Möbius, se define el espacio de Bloch y su relación con la proyección de Bergman, además se define la transformada de Berenzin. Dentro de este capítulo se da una prueba directa y original de la igualdad entre el espacio pequeño de Bloch $\mathcal{B}_{0}$ y el espacio $V M O(\mathbb{D})$, que consiste de las funciones analíticas que se desvanecen en la frontera.

En el tercer capítulo se definen los espacios pesados de Bergman ${ }_{s} \mathcal{A}_{q}^{p}, L(p, q, s)$ y los espacios $\alpha$-growth. Además, para $1<s<\infty$ se da una caracterización de los espacios $\alpha$-growth, la cual está estrechamente relacionada con los espacios ${ }_{s} \mathcal{A}_{q}^{p}$ y $L(p, q, s)$. También se prueba la igualdad ente los espacios ${ }_{s} \mathcal{A}_{q}^{p}$ y $L(p, q, s)$. Se estudian algunas de sus propiedades tales como la Möbius invarianza, el anidamiento de inclusiones propias y se dan caracterizaciones alternativas. Además se define una norma para ${ }_{s} \mathcal{A}_{q}^{p}$ con $1 \leq p<\infty$, la cual es inducida por su definición, y con ella los espacios ${ }_{s} \mathcal{A}_{q}^{p}$ son completos. Para $0<p<1$ se obtiene una métrica inducida por su definición, donde nuevamente los espacios son completos. 
Finalmente en el cuarto capítulo se estudia el operador de Bergman $P_{\beta}$ del espacio $L^{p}\left(\mathbb{D}, d A_{q}\right)$ en los espacios de Bergman pesados ${ }_{s} \mathcal{A}_{q}^{p}=L(p, q, s)$ y se prueba que es un operador acotado para ciertos valores de $\beta, p, q$ y $s$ que en particular satisfacen la relación $q+1 \geq(\beta+1) p$ y así un bien conocido Teorema (Teorema 1.5.5) se puede extender en el sentido de la suficiencia, gracias a la adición del peso $1-\left|\varphi_{z}(w)\right|^{2}$. 


\section{Introducción}

La teoría de los espacios de funciones analíticas ha recibido un fuerte impulso en su estudio durante las últimas décadas. Ejemplos clásicos de tales espacios son los espacios de Hardy, los espacios de Bergman y los espacios de Bloch. Más recientemente los espacios de Besov, los espacios pesados de Dirichlet y la teoría BMO han sido estudiadas intensamente, mientras la teoría de espacios tales como los espacios $\alpha$-growth y los espacios $Q_{p}$ se siguen estudiando ampliamente. Todos estos espacios de funciones son interesantes por si solos y además tienen interesantes relaciones con otros campos de la matemática como el análisis funcional, ecuaciones diferenciales, teoría de potencial, teoría de operadores, entre otras.

El estudio de los espacios de Bergman se remonta al año 1950, cuando Stefan Bergman publicó su monografía titulada The Kernel Function and Conformal Mapping, en el cual desarrolló la teoría de los espacios de Hilbert de funciones analíticas sobre un dominio $\Omega$ en el plano. El trabajo de Bergman se centró en el estudio del espacio de las funciones analíticas, cuadrado integrables con respecto a la medida de área de Lebesgue en un dominio $\Omega$. Esta teoría se apoyó en la existencia de una función núcleo reproductor, la cual hoy en día es conocida como la función núcleo de Bergman.

Partiendo de la definición de los espacios de Bergman es natural definir los espacios pesados de Bergman. Más precisamente, sea $\mathbb{D}$ el disco unitario de $\mathbb{C}$. Para $0<p<$ $\infty$ y $-1<\alpha<\infty$ se define $A_{\alpha}^{p}(\mathbb{D})$ como el espacio de funciones analíticas $f$ en $L^{p}\left(\mathbb{D}, d A_{\alpha}\right)$ tales que

$$
\int_{\mathbb{D}}|f(z)|^{p} d A_{\alpha}(z)<\infty,
$$

donde

$$
d A_{\alpha}(z)=(\alpha+1)\left(1-|z|^{2}\right)^{\alpha} d A(z),
$$

y $d A(z)$ es la medida de Lebesgue en el disco unitario.

Entonces el espacio de las funciones analíticas $p$ integrables, $0<p<\infty$, sobre el disco 
unitario $\mathbb{D}$, con la medida $d A_{\alpha}(z)$ es precisamente el Espacio de Bergman Pesado $\mathcal{A}_{\alpha}^{p}(\mathbb{D})$.

Dentro de la teoría de los espacios de Bergman, es de especial interés el caso $p=2$. Así el conjunto $A_{\alpha}^{2}(\mathbb{D})$ es un espacio de Hilbert con el producto interno definido por

$$
\langle f, g\rangle_{\alpha}:=\int_{\mathbb{D}} f(z) \overline{g(z)} d A_{\alpha}(z) .
$$

Como el espacio de Bergman $A_{\alpha}^{2}(\mathbb{D})$ es un subespacio cerrado de $L^{2}\left(\mathbb{D}, d A_{\alpha}\right)$, existe un único operador de proyección

$$
P_{\alpha}: L^{2}\left(\mathbb{D}, d A_{\alpha}\right) \longrightarrow A_{\alpha}^{2}(\mathbb{D}),
$$

lineal y continuo que satisface $P_{\alpha}(f)=f$ para toda $f \in A_{\alpha}^{2}(\mathbb{D})$. Además se puede dar una fórmula explícita para la proyección $P_{\alpha}$ y está dada por

$$
P_{\alpha} f(z)=\int_{\mathbb{D}} \frac{f(w)}{(1-z \bar{w})^{2+\alpha}} d A_{\alpha}(w), \quad z \in \mathbb{D},
$$

para toda $f \in L^{2}\left(\mathbb{D}, d A_{\alpha}\right)$. Como $A_{\alpha}^{2}(\mathbb{D}) \subset A_{\alpha}^{1}(\mathbb{D})$ y además $A_{\alpha}^{2}(\mathbb{D})$ es denso en $A_{\alpha}^{1}(\mathbb{D})$ se puede extender el dominio de $P_{\alpha}$ a $L^{1}\left(\mathbb{D}, d A_{\alpha}\right)$, donde la integral dada por (1) converge uniformemente para $z$ en cada subconjunto compacto de $\mathbb{D}$. En particular se puede aplicar $P_{\alpha}$ a una función en $L^{p}\left(\mathbb{D}, d A_{\alpha}\right)$ siempre que $1 \leq p<\infty$. Al operador $P_{\alpha}$ se le conoce como Proyección de Bergman sobre $A_{\alpha}^{p}(\mathbb{D})$.

Otros espacios de interés son el espacio de Bloch, denotado por $\mathcal{B}$, el cual está compuesto por las funciones analíticas $f$ en $\mathbb{D}$ que satisfacen la siguiente condición,

$$
\sup _{z \in \mathbb{D}}\left(1-|z|^{2}\right)\left|f^{\prime}(z)\right|<+\infty
$$

y el espacio pequeño de Bloch, el cual es denotado por $\mathcal{B}_{0}$ y es el subespacio de $\mathcal{B}$ consistente de todas las funciones $f \in \mathcal{B}$ tales que

$$
\lim _{|z| \rightarrow 1^{-}}\left(1-|z|^{2}\right)\left|f^{\prime}(z)\right|=0 .
$$

Estos espacios han sido estudiados por si sólos y tienen una particular relevancia pues están relacionados con la proyección de Bergman (ver Teorema 1.12 [HeKoZh]). Además resulta ser que el espacio de Bloch es el espacio dual del espacio de Bergman pesado $A_{\alpha}^{p}$ cuando $0<p \leq 1$ y $-1<\alpha<\infty$. 
Por otra parte, sean $p, q$ y $s$ tales que $0<p<\infty,-2<q<\infty$ y $0<s<\infty$. Una función analítica $f: \mathbb{D} \longrightarrow \mathbb{C}$ definida en el disco unitario $\mathbb{D}$ pertenece a el espacio ${ }_{s} \mathcal{A}_{q}^{p}$, si

$$
\sup _{z \in \mathbb{D}} \int_{\mathbb{D}}|f(z)|^{p} g^{s}(z, w) d A_{q}(z)<\infty
$$

donde $g^{s}(z, w)=\log \left|\frac{1-\bar{w} z}{z-w}\right|$ es la función de Green de $\mathbb{D}$ con singularidad logarítmica en $w \in \mathbb{D}$.

En este trabajo se estudian los espacios ${ }_{s} \mathcal{A}_{q}^{p}$ usando técnicas del análisis real y complejo, donde las tranformaciones de Möbius y la función de Green en el disco unitario juegan un papel preponderante, así como ciertas estimaciones integrales que resultan cruciales en esta teoría. 


\section{Capítulo 1}

\section{Algunos operadores integrales en los espacios $L^{p}$}

En este capítulo se enuncian definiciones y resultados conocidos sobre operadores lineales en espacios de Banach. Una referencia para resultados en este capítulo es [HeKoZh].

\subsection{Operadores acotados en espacios de Banach}

Sea $\mathbb{C}$ el campo de los números complejos. Un espacio de Banach $X$ es un espacio lineal (vectorial) complejo con una función no negativa \|| || la cual satisface las siguientes condiciones:

(1) $\|x+y\| \leq\|x\|+\|y\|$ para todo $x, y$ en $X$.

(2) $\|a x\|=|a|\|x\|$ para todo $x \in X$ y todo $a \in \mathbb{C}$.

(3) $\|x\|=0$ si y sólo si $x=0$.

(4) Si $\left\{x_{n}\right\}$ es una sucesión de Cauchy en $X$, entonces existe $x \in X$ tal que $\left\|x_{n}-x\right\| \rightarrow 0$ cuando $n \rightarrow \infty$.

La función \|| || se llama norma en $X$ y un espacio lineal que satisface sólo las condiciones (1), (2) y (3) se llama espacio normado. La condición (4) caracteriza a la completez del espacio $X$.

Sean $X$ y $Y$ espacios de Banach y $T: X \rightarrow Y$ un operador lineal. Se dice que $T$ es un operador lineal acotado de $X$ sobre $Y$ si existe una constante $C>0$ tal que

$$
\|T x\| \leq C\|x\|, \quad \text { para toda } x \in X .
$$


Teorema 1.1.1 Sea $T: \mathcal{D}(T) \rightarrow Y$ un operador lineal, donde $\mathcal{D}(T) \subset X$ y $X, Y$ son espacios normados, entonces $T$ es un operador lineal acotado si y sólo si es $T$ es un operador lineal continuo.

\subsection{Desigualdad de Hölder}

Teorema 1.2.1 Supóngase que $p, q>1$ con $p^{-1}+q^{-1}=1$ y que $\mu$ es una medida positiva en $X$. Si $f \in L^{p}(X, d \mu)$ y $g \in L^{q}(X, d \mu)$, entonces $f g \in L^{1}(X, d \mu)$ y

$$
\left|\int_{X} f \bar{g} d \mu\right| \leq\left(\int_{X}|f|^{p} d \mu\right)^{1 / p}\left(\int_{X}|g|^{q} d \mu\right)^{1 / q} .
$$

En particular, si $\mu$ es una medida de probabilidad, entonces

$$
\left|\int_{X} f d \mu\right| \leq\left(\int_{X}|f|^{p} d \mu\right)^{1 / p}
$$

para toda $p>1$ y $f \in L^{p}(X, d \mu)$.

\subsection{Teorema de Schur}

Sean $(X, \mu)$ un espacio de medida y $T: X \times X \rightarrow \mathbb{R}$ una función. Sea $\mathbf{T}$ el operador integral con núcleo $T$ dado por

$$
\mathbf{T} f(x)=\int_{X} T(x, y) f(y) d \mu(y),
$$

donde $f: X \rightarrow \mathbb{R}$ es una función medible.

En esta sección se presentan condiciones suficientes sobre $T$ para la acotación del operador integral asociado $\mathbf{T}$ en $L^{p}(X, d \mu)$ con $1<p<+\infty$. Este resultado se llama Teorema de Schur.

Teorema 1.3.1 Supóngase que $(X, \mu)$ es un espacio medible con $\mu$ una medida positiva. Sea $T(x, y)$ una función medible positiva en $X \times X$ y $\mathbf{T}$ el operador integral inducido

$$
\mathbf{T} f(x)=\int_{X} T(x, y) f(y) d \mu(y), \quad x \in X,
$$

definido si la integral converge. Si para algún $1<p<\infty$ existe una función h medible, estrictamente positiva en $X$ y una constante positiva $M>0$ tal que

$$
\int_{X} T(x, y) h(y)^{q} d \mu(y) \leq M h(x)^{q}, \quad x \in X,
$$


$y$

$$
\int_{X} T(x, y) h(x)^{p} d \mu(x) \leq M h(y)^{p}, \quad y \in X,
$$

con $p^{-1}+q^{-1}=1$, entonces $\mathbf{T}$ es acotado en $L^{p}(X, d \mu)$ con $\|\mathbf{T}\| \leq M$.

\subsection{Operadores integrales en el disco unitario $\mathbb{D}$}

Sea $0<r<\infty$. Se define $\mathbb{D}_{r}=\{z \in \mathbb{C}:|z|<r\}$. Se denotará por $\mathbb{D}=\mathbb{D}_{1}$ al disco unitario abierto en el plano complejo $\mathbb{C}$ y al anillo $r<|z|<1$ por $\mathbb{A}_{r}=\mathbb{D} \backslash \mathbb{D}_{r}$.

Se denota por $d A$ la medida normalizada de Lebesgue en $\mathbb{D}$, la cual está dada por

$$
d A(z)=\frac{1}{\pi} d x d y=\frac{1}{\pi} r d r d \theta
$$

donde $z=x+i y=r e^{i \theta}$. También se define para $\alpha \in \mathbb{R}$ la medida

$$
d A_{\alpha}(z):=(\alpha+1)\left(1-|z|^{2}\right)^{\alpha} d A(z) .
$$

Lema 1.4.1 Sea $\alpha>-1$. Entonces

$$
\int_{\mathbb{D}} d A_{\alpha}(z)=1
$$

Se dice que dos cantidades $A$ y $B$ son comparables (y se denotará por $\sim$ ) si existe una constante $C$, independiente de $A$ y $B$, tal que

$$
\frac{A}{C} \leq B \leq A C
$$

Las siguientes estimaciones integrales son fundamentales en el estudio de operadores sobre espacios de funciones en el disco unitario (ver [HeKoZh]).

Teorema 1.4.2 [Estimación Integral] Sean $-1<t<\infty$ y $c \in \mathbb{R}$. Se definen

$$
I_{t, c}(z):=\int_{\mathbb{D}} \frac{\left(1-|w|^{2}\right)^{t}}{|1-z \bar{w}|^{2+t+c}} d A(w), \quad z \in \mathbb{D},
$$

$y$

$$
J_{c}(z):=\int_{0}^{2 \pi} \frac{d \theta}{\left|1-z e^{-i \theta}\right|^{1+c}}, \quad z \in \mathbb{D}
$$


Entonces se tiene la relación de comparabilidad

$$
I_{t, c}(z) \sim J_{c}(z) \sim \begin{cases}1 & \text { si } c<0 \\ \log \frac{1}{1-|z|^{2}} & \text { si } c=0 \\ \frac{1}{\left(1-|z|^{2}\right)^{c}} & \text { si } c>0\end{cases}
$$

cuando $|z| \rightarrow 1^{-}$.

El siguiente teorema dice cuando son acotados en $L^{p}(\mathbb{D}, d \mu)$ los siguientes operadores definidos en él.

Teorema 1.4.3 Supóngase que a,b,c son números reales y $\mathbf{T}$ y $\mathbf{S}$ los operadores integrales definidos por

$$
\mathbf{T} f(z)=\left(1-|z|^{2}\right)^{a} \int_{\mathbb{D}} \frac{\left(1-|w|^{2}\right)^{b}}{(1-z \bar{w})^{2+a+b}} f(w) d A(w)
$$

$y$

$$
\mathbf{S} f(z)=\left(1-|z|^{2}\right)^{a} \int_{\mathbb{D}} \frac{\left(1-|w|^{2}\right)^{b}}{|1-z \bar{w}|^{2+a+b}} f(w) d A(w)
$$

Entonces para $1 \leq p<\infty$ las siguientes condiciones son equivalentes:

(1) $\mathbf{T}$ es acotado en $L^{p}\left(\mathbb{D}, d A_{c}\right)$.

(2) $\mathbf{S}$ es acotado en $L^{p}\left(\mathbb{D}, d A_{c}\right)$.

(3) $-p a<c+1<p(b+1)$.

\subsection{Espacios de Bergman}

En esta sección se definen los Espacios clásicos de Bergman en el disco unitario $\mathbb{D}$ y la proyección de Bergman en $L^{p}\left(\mathbb{D}, d A_{\alpha}\right)$.

Para $0<p<\infty$ y $-1<\alpha<+\infty$ se define el espacio clásico de Bergman $A_{\alpha}^{p}=A_{\alpha}^{p}(\mathbb{D})$ del disco unitario, como el conjunto de las funciones analíticas en $L^{p}\left(\mathbb{D}, d A_{\alpha}\right)$. 
Si $f$ está en $L^{p}\left(\mathbb{D}, d A_{\alpha}\right)$, se escribe

$$
\|f\|_{p, \alpha}=\left(\int_{\mathbb{D}}|f(z)|^{p} d A_{\alpha}(z)\right)^{\frac{1}{p}}
$$

en el caso en que $\alpha=0$ simplemente se escribe $\|f\|_{p}=\|f\|_{p, 0}$.

Se observa que si $1 \leq p<\infty$, el espacio $L^{p}\left(\mathbb{D}, d A_{\alpha}\right)$ es un espacio de Banach con la norma definida por $\|f\|_{p, \alpha}$ y si $0<p<1$, el espacio $L^{p}\left(\mathbb{D}, d A_{\alpha}\right)$ es un espacio métrico completo con la métrica definida por

$$
d(f, g)=\|f-g\|_{p, \alpha}^{p} .
$$

Se denota por $L^{\infty}(\mathbb{D})$ el espacio de todas las funciones esencialmente acotadas en $\mathbb{D}$ y para $f \in L^{\infty}(\mathbb{D})$, se define

$$
\|f\|_{\infty}=\operatorname{supess}\{|f(z)|: z \in \mathbb{D}\} .
$$

El espacio $L^{\infty}(\mathbb{D})$ es un espacio de Banach con la norma $\|\cdot\|_{\infty}$. El subespacio de $L^{\infty}$ formado por todas las funciones analíticas acotadas en $\mathbb{D}$ se denota por $H^{\infty}$. Debido a la convergencia uniforme, $H^{\infty}$ es un subespacio cerrado de $L^{\infty}(\mathbb{D})$ y por ello es un espacio de Banach.

El siguiente resultado dice que las funciones en los espacios de Bergman y sus derivadas tienen un crecimiento acotado sobre subconjuntos compactos de $\mathbb{D}$.

Proposición 1.5.1 Supóngase que $0<p<\infty,-1<\alpha<+\infty$ y que $K$ es un subconjunto compacto de $\mathbb{D}$. Entonces existe una constante positiva $C=C(n, K, p, \alpha)$ tal que

$$
\sup \left\{\left|f^{(n)}(z)\right|: z \in K\right\} \leq C\|f\|_{p, \alpha}
$$

para toda $f \in A_{\alpha}^{p}$ y toda $n \in \mathbb{N} \cup\{0\}$. En particular cada evaluación puntual en $\mathbb{D}$ $T_{z}(f)=f(z)$, es un funcional lineal acotado en $A_{\alpha}^{p}$ con $p \geq 1$.

La proposición anterior tiene una consecuencia muy importante: el espacio $A_{\alpha}^{p}$ es cerrado en $L^{p}\left(\mathbb{D}, d A_{\alpha}\right)$.

Proposición 1.5.2 Para cada $0<p<\infty y-1<\alpha<+\infty$ el espacio de Bergman $A_{\alpha}^{p}$ es cerrado en $L^{p}\left(\mathbb{D}, d A_{\alpha}\right)$.

Demostración. Sea $\left\{f_{n}\right\}$ una sucesión en $A_{\alpha}^{p}$ y supóngase que $f_{n} \rightarrow f$ en $L^{p}\left(\mathbb{D}, d A_{\alpha}\right)$. En particular $\left\{f_{n}\right\}$ es una sucesión de Cauchy en $L^{p}\left(\mathbb{D}, d A_{\alpha}\right)$. Sin pérdida de generalidad considérese el disco compacto $K=\{z \in \mathbb{D}:|z| \leq r\}$ con $0<r<1$. Así, por la Proposición 1.5.1 existe $M>0$ tal que

$$
\left|f_{n}(z)-f_{m}(z)\right| \leq M\left\|f_{n}-f_{m}\right\|_{p, \alpha}
$$


para toda $z \in K$. Por lo tanto la sucesión $\left\{f_{n}\right\}$ converge uniformemente en cada subconjunto compacto de $\mathbb{D}$. Por el Teorema de Montel $f$ es analítica en $\mathbb{D}$, luego pertenece a $A_{\alpha}^{p}$.

Como consecuencia de la Proposición 1.5.2 el espacio de Bergman $A_{\alpha}^{p}$ es un espacio de Banach cuando $1 \leq p<\infty$ y es un espacio métrico completo cuando $0<p<1$.

La Proposición 1.5.2 implica que $A_{\alpha}^{2}$ es un espacio de Hilbert con el producto interno dado por

$$
\langle f, g\rangle_{\alpha}=\int_{\mathbb{D}} f(z) \overline{g(z)} d A_{\alpha}(z),
$$

donde $f, g \in A_{\alpha}^{2}$. Para cualquier número $n$ no negativo, sea

$$
e_{n}=\sqrt{\frac{\Gamma(n+2+\alpha)}{n ! \Gamma(2+\alpha)}} z^{n}, \quad z \in \mathbb{D},
$$

donde $\Gamma(s)$ representa la función Gamma, la cual es una función analítica de $s$ en todo el plano complejo, excepto en sus polos simples localizados en los puntos $\{0,-1,-2, \ldots\}$. El conjunto $\left\{e_{n}\right\}$ es una base ortonormal para el espacio $A_{\alpha}^{2}$.

Ahora como el espacio $A_{\alpha}^{2}$ es un subespacio cerrado de $L^{2}\left(\mathbb{D}, d A_{\alpha}\right)$, existe el operador de proyección ortogonal $P_{\alpha}: L^{2}\left(\mathbb{D}, d A_{\alpha}\right) \rightarrow A_{\alpha}^{2}$ lineal y continuo tal que $P_{\alpha} f=f$, si $f \in A_{\alpha}^{2}(\mathbb{D})$. En este caso se puede dar la forma explícita de dicha proyección, como se ve en la siguiente proposición.

Proposición 1.5.3 Para $-1<\alpha<+\infty$, sea $P_{\alpha}$ la proyección ortogonal de $L^{2}\left(\mathbb{D}, d A_{\alpha}\right)$ sobre $A_{\alpha}^{2}$. Entonces

$$
P_{\alpha} f(z)=\int_{\mathbb{D}} \frac{f(w)}{(1-z \bar{w})^{2+\alpha}} d A_{\alpha}(w), \quad z \in \mathbb{D},
$$

para todo $f \in L^{2}\left(\mathbb{D}, d A_{\alpha}\right)$.

Demostración. Sea $\left\{e_{n}\right\}$ la base ortonormal de $A_{\alpha}^{2}$ dada por (1.4). Para cada $f \in$ $L^{2}\left(\mathbb{D}, d A_{\alpha}\right)$ se tiene que

$$
P_{\alpha} f=\sum_{n=0}^{\infty}\left\langle P_{\alpha} f, e_{n}\right\rangle_{\alpha} e_{n} .
$$

En particular para cada $z \in \mathbb{D}$ se tiene

$$
P_{\alpha} f(z)=\sum_{n=0}^{\infty}\left\langle P_{\alpha} f, e_{n}\right\rangle_{\alpha} e_{n}(z),
$$


y esta serie converge uniformemente en subconjuntos compactos de $\mathbb{D}$. Además

$$
\left\langle P_{\alpha} f, e_{n}\right\rangle_{\alpha}=\left\langle f, P_{\alpha} e_{n}\right\rangle_{\alpha}=\left\langle f, e_{n}\right\rangle_{\alpha}
$$

Por lo tanto

$$
\begin{aligned}
P_{\alpha} f(z) & =\sum_{n=0}^{\infty}\left\langle P_{\alpha} f, e_{n}\right\rangle_{\alpha} e_{n}(z)=\sum_{n=0}^{\infty}\left\langle f, e_{n}\right\rangle_{\alpha} e_{n}(z) \\
& =\sum_{n=0}^{\infty} \int_{\mathbb{D}}\left(f(w) \sqrt{\frac{\Gamma(n+2+\alpha)}{n ! \Gamma(2+\alpha)}} \bar{w}^{n}\right) d A_{\alpha}(w) \sqrt{\frac{\Gamma(n+2+\alpha)}{n ! \Gamma(2+\alpha)}} z^{n} \\
& =\sum_{n=0}^{\infty} \frac{\Gamma(n+2+\alpha)}{n ! \Gamma(2+\alpha)} \int_{\mathbb{D}} f(w)(z \bar{w})^{n} d A_{\alpha}(w) \\
& =\int_{\mathbb{D}} f(w)\left[\sum_{n=0}^{\infty} \frac{\Gamma(n+2+\alpha)}{n ! \Gamma(2+\alpha)}(z \bar{w})^{n}\right] d A_{\alpha}(w) \\
& =\int_{\mathbb{D}} \frac{f(w)}{(1-z \bar{w})^{2+\alpha}} d A_{\alpha}(w) .
\end{aligned}
$$

Se pueden intercambiar la integral y la suma ya que para cada $z \in \mathbb{D}$ fijo la serie $\sum_{n=0}^{\infty} \frac{\Gamma(n+2+\alpha)}{n ! \Gamma(2+\alpha)}(z \bar{w})^{n}$ converge uniformemente con respecto a $w \in \mathbb{D}$.

Si $f \in A_{\alpha}^{2}$ entonces

$$
P_{\alpha} f(z)=f(z)=\int_{\mathbb{D}} \frac{f(w)}{(1-z \bar{w})^{2+\alpha}} d A_{\alpha}(w) .
$$

El operador $P_{\alpha}$ se llama la proyección de Bergman en $\mathbb{D}$ y la función

$$
K_{\alpha}(z, w)=\frac{1}{(1-z \bar{w})^{2+\alpha}}, \quad z, w \in \mathbb{D}
$$

se llama el núcleo reproductor de Bergman sobre $\mathbb{D}$, el cual es holomorfo con respecto a la variable $z$ y anti-holomorfo con respecto a la variable $w$. Esta función juega un papel muy relevante en la teoría de los espacios de Bergman.

Aunque la proyección de Bergman $P_{\alpha}$ está originalmente definida en $L^{2}\left(\mathbb{D}, d A_{\alpha}\right)$, la fórmula integral

$$
P_{\alpha} f(z)=\int_{\mathbb{D}} \frac{f(w)}{(1-z \bar{w})^{2+\alpha}} d A_{\alpha}(w)
$$


extiende su dominio a $L^{1}\left(\mathbb{D}, d A_{\alpha}\right)$. En particular, se puede aplicar $P_{\alpha}$ a una función en $L^{p}\left(\mathbb{D}, d A_{\alpha}\right)$ con $1 \leq p<\infty$.

Como $A_{\alpha}^{2}$ es denso en $A_{\alpha}^{1}$, el siguiente resultado justifica plenamente la definición de núcleo reproductor.

Corolario 1.5.4 Sif es una función en $A_{\alpha}^{1}$, entonces

$$
f(z)=\int_{\mathbb{D}} \frac{f(w)}{(1-z \bar{w})^{2+\alpha}} d A_{\alpha}(w), \quad z \in \mathbb{D} .
$$

La integral converge uniformemente para $z$ en cada subconjunto compacto de $\mathbb{D}$. El corolario anterior se conoce como la fórmula reproductora.

El conjunto $A^{p}=A_{0}^{p}$ es el espacio ordinario de Bergman, su correspondiente proyección de Bergman se denota por $P$ y su núcleo de Bergman es

$$
K(z, w)=\frac{1}{(1-z \bar{w})^{2}} .
$$

Las funciones núcleo de Bergman están relacionadas con las transformaciones de Möbius del disco. Las propiedades de dichas transformaciones se estudiarán en el siguiente capítulo. Para ver la relación anteriormente mencionada, sea $z \in \mathbb{D}$ y se considera la transformación $\varphi_{z}$ del disco sobre si mismo que intercambia $z$ y 0

$$
\varphi_{z}(w)=\frac{z-w}{1-\bar{z} w}, \quad w \in \mathbb{D} .
$$

La igualdad

$$
\varphi_{z}^{\prime}(w)=\frac{\left(1-|z|^{2}\right)}{(1-z \bar{w})^{2}}=\left(1-|z|^{2}\right) K(z, w) .
$$

Si $f \in A_{\alpha}^{1}$, por la propiedad del valor medio se tiene

$$
f(0)=\frac{1}{2 \pi} \int_{0}^{2 \pi} f\left(r e^{i \theta}\right) d \theta,
$$

entonces

$$
\begin{aligned}
\int_{0}^{1} f(0)\left(1-r^{2}\right)^{\alpha} r d r & =\frac{1}{2 \pi} \int_{0}^{2 \pi} f\left(r e^{i \theta}\right) d \theta \int_{0}^{1}\left(1-r^{2}\right)^{\alpha} r d r \\
& =\frac{1}{2 \pi} \int_{0}^{1} \int_{0}^{2 \pi} f\left(r e^{i \theta}\right)\left(1-r^{2}\right)^{\alpha} r d \theta d r \\
& =\frac{1}{2(\alpha+1)} \int_{\mathbb{D}} f(w) d A_{\alpha}(w)
\end{aligned}
$$


Como

$$
\int_{0}^{1} f(0)\left(1-r^{2}\right)^{\alpha} r d r=\frac{1}{2(\alpha+1)} f(0)
$$

se obtiene

$$
f(0)=\int_{\mathbb{D}} f(w) d A_{\alpha}(w) .
$$

Ahora sí se reemplaza $f$ por $f \circ \varphi_{z}$ en (1.5)

$$
\left(f \circ \varphi_{z}\right)(0)=\int_{\mathbb{D}}\left(f \circ \varphi_{z}\right)(w) d A_{\alpha}(w)
$$

y tomando el cambio de variable dado por $w=\varphi_{z}(\lambda)$ y aplicando las propiedades (2) y (3) de la Proposición 2.1.1

$$
\begin{aligned}
f(z) & =(\alpha+1) \int_{\mathbb{D}} f(\lambda)\left(1-\left|\varphi_{z}(\lambda)\right|^{2}\right)^{\alpha} \frac{\left(1-|z|^{2}\right)^{2}}{|1-z \bar{\lambda}|^{4}} d A(\lambda) \\
& =\left(1-|z|^{2}\right)^{2+\alpha} \int_{\mathbb{D}} \frac{f(\lambda)}{|1-z \bar{\lambda}|^{2(2+\alpha)}} d A_{\alpha}(\lambda) .
\end{aligned}
$$

Cambiando $\lambda$ por $w$ se tiene

$$
f(z)=\left(1-|z|^{2}\right)^{2+\alpha} \int_{\mathbb{D}} \frac{f(w)}{(1-z \bar{w})^{2+\alpha}(1-\bar{z} w)^{2+\alpha}} d A_{\alpha}(w) .
$$

Fijando $z \in \mathbb{D}$, y reemplazando $f$ por la función $w \mapsto(1-\bar{z} w)^{2+\alpha} f(w)$, entonces se tendra nuevamente la fórmula reproductora

$$
f(z)=\int_{\mathbb{D}} \frac{f(w)}{(1-z \bar{w})^{2+\alpha}} d A_{\alpha}(w), \quad z \in \mathbb{D},
$$

para $f \in A_{\alpha}^{1}$. De esto es inmediato deducir la fórmula integral para la proyección de Bergman.

El siguiente teorema dice que existe un gran número de proyecciones acotadas de $L^{1}(\mathbb{D}, d A)$ sobre el espacio de Bergman $A^{1}$.

Teorema 1.5.5 Supóngase que $-1<\alpha, \beta<\infty$ y $1 \leq p<\infty$. Entonces $P_{\beta}$ es una proyección acotada de $L^{p}\left(\mathbb{D}, d A_{\alpha}\right)$ sobre $A_{\alpha}^{p}$ si y sólo si $\alpha+1<(\beta+1)$ p.

Demostración. Para probar esta equivalencia sea $d \mu(z)=\left(1-|z|^{2}\right)^{\alpha} d A(z)$, así pues aplicando el Teorema 1.4.3 tenemos que el operador $P_{\beta}:\left(L^{p}(\mathbb{D}), d A_{\alpha}\right) \rightarrow A_{\alpha}^{p}(\mathbb{D})$ es acotado si y sólo si $\alpha+1<p(\beta+1)$.

Dos casos importantes de mencionar son: 
1. Si $\alpha=\beta$, entonces $P_{\alpha}$ es una proyección acotada de $L^{p}\left(\mathbb{D}, d A_{\alpha}\right)$ sobre $A_{\alpha}^{p}$ si y sólo si $1<p<\infty$.

2. Si $p=1$, entonces $P_{\beta}$ es una proyección acotada de $L^{1}\left(\mathbb{D}, d A_{\alpha}\right)$ sobre $A_{\alpha}^{1}$ si y sólo si $\alpha<\beta$. 


\section{Capítulo 2}

\section{El Espacio $V M O(\mathbb{D})$}

En este capítulo se dan propiedades generales de las transformaciones de Möbius, se define el espacio de Bloch y su relación con la proyección de Bergman y además se define la transformada de Berezin y al espacio $V M O(\mathbb{D})$, de las funciones analíticas que se desvanecen en la frontera. El material de este capítulo puede ser consultado en el Libro Theory of Bergman Spaces, escrito por Haakan Hedenmalm, Boris Korenblum y Kehe Zhu. En dicho libro y las referencias [Duren], [Zhu2] y [KeZh] no se la prueba completa de la igualdad entre los espacios pequeño de Bloch $\mathcal{B}_{0}$ y $V M O(\mathbb{D})$, en cada una de ellas sólo se muestra que $V M O(\mathbb{D}) \cap H(\mathbb{D}) \subset \mathcal{B}_{0}$. Así pues la aportación más importante en este capítulo es dar una prueba directa y original de que $V M O(\mathbb{D}) \cap H(\mathbb{D})=\mathcal{B}_{0}$. Para ello en la Sección 2.4 se presentan dos estimaciones tipo Littlewood-Paley que son completamente originales y son pieza clave para dar la demostración directa de dicha igualdad. Así mismo se explican todos los cálculosrelativos a la prueba, en particular los que involucran propiedades que satisface la transformada de Berezin.

\subsection{Transformaciones de Möbius}

Se considera la familia de transformaciones de Möbius $\varphi_{z}: \mathbb{C} \backslash\left\{\frac{1}{\bar{z}}\right\} \rightarrow \mathbb{C}$ dadas por

$$
\varphi_{z}(w)=\frac{z-w}{1-\bar{z} w},
$$

con polo en $w=1 / \bar{z}$. Las transformaciones de Möbius $\varphi_{z}$ tienen las siguientes propiedades.

Proposición 2.1.1 La función $\varphi_{z}$ satisface

(1) $\varphi_{z}=\varphi_{z}^{-1}$. 
(2) El determinante del Jacobiano de $\varphi_{z}$ en $w$ es

$$
\left|\varphi_{z}^{\prime}(w)\right|^{2}=\frac{\left(1-|z|^{2}\right)^{2}}{|1-\bar{z} w|^{4}}
$$

(3) $1-\left|\varphi_{z}(w)\right|^{2}=\frac{\left(1-|z|^{2}\right)\left(1-|w|^{2}\right)}{|1-\bar{z} w|^{2}}=\left(1-|w|^{2}\right)\left|\varphi_{z}^{\prime}(w)\right|$.

(4) $\left|1-\varphi_{z}(w) \bar{z}\right|=\frac{1-|z|^{2}}{|1-\bar{z} w|}$.

(5) Si $z \in \mathbb{D}$ entonces $\varphi_{z}$ es una función biholomorfa del disco unitario sobre si mismo.

(6) Sean $q \in \mathbb{R} y z \in \mathbb{D}$. Entonces para todo $w \in \mathbb{D}$

$$
\frac{1}{\rho(z, q)}\left(1-|w|^{2}\right)^{q} \leq\left(1-\left|\varphi_{z}(w)\right|^{2}\right)^{q} \leq \rho(z, q)\left(1-|w|^{2}\right)^{q}
$$

$y$

$$
\frac{1}{\rho(z, q)} \leq\left|\varphi_{z}^{\prime}(w)\right|^{q} \leq \rho(z, q)
$$

donde

$$
\rho(z, q)=\left(\frac{1+|z|}{1-|z|}\right)^{|q|}
$$

El conjunto $\left\{\varphi_{z}: z \in \mathbb{D}\right\}$ no es un subgrupo de las transformaciones de Möbius.

La siguiente fórmula de cambio de variable es consecuencia de la propiedad (2).

Proposición 2.1.2 Si $f \in L^{1}(\mathbb{D}, d A)$, entonces

$$
\int_{\mathbb{D}} f \circ \varphi_{z}(w) d A(w)=\int_{\mathbb{D}} f(w) \frac{\left(1-|z|^{2}\right)^{2}}{|1-\bar{z} w|^{4}} d A(w) .
$$

Más generalmente se tiene la siguiente fórmula de cambio de variable.

Proposición 2.1.3 Si $f \in L^{1}\left(\mathbb{D}, d A_{\alpha}\right)$ y $\alpha>-1$, entonces

$$
\int_{\mathbb{D}} f \circ \varphi_{z}(w) d A_{\alpha}(w)=\int_{\mathbb{D}} f(w) \frac{\left(1-|z|^{2}\right)^{2+\alpha}}{|1-\bar{z} w|^{2(2+\alpha)}} d A(w) .
$$

Sea $0<r<1$. El disco seudohiperbólico está definido por

$$
\mathcal{D}(z, R):=\varphi_{z}\left(\mathbb{D}_{R}\right)=\left\{w \in \mathbb{D}:\left|\varphi_{z}(w)\right|<R\right\}
$$


De hecho $\mathcal{D}(z, R)$ es un disco Euclideano con centro $c$ y radio $r$ dados por

$$
c=\frac{1-R^{2}}{1-R^{2}|z|^{2}} z, \quad r=\frac{1-|z|^{2}}{1-R^{2}|z|^{2}} R
$$

y se denota por $|\mathcal{D}(z, R)|$ su área.

Proposición 2.1.4 Sean $0<r<1$ y $0<R<1$. Entonces existe $\rho>0$ tal que si $\rho<|z|<1$, se cumple

$$
D(z, R) \subset \mathbb{D} \backslash \mathbb{D}_{r} .
$$

Para $z, w \in \mathbb{D}$ la función de Green de $\mathbb{D}$, con singularidad logarítmica en $z$, es

$$
g(w, z)=\ln \frac{|1-\bar{z} w|}{|z-w|}=\ln \frac{1}{\left|\varphi_{z}(w)\right|} .
$$

Lema 2.1.5 Sean $-2<q<\infty, 0<s<\infty y-1<q+s$. Entonces

$$
\begin{aligned}
\lim _{|z| \rightarrow 1^{-}} \int_{\mathbb{D}}\left(1-\left|\varphi_{z}(w)\right|^{2}\right)^{s} d A_{q}(w) & =0, \\
\lim _{|z| \rightarrow 1^{-}} \int_{\mathbb{D}} g^{s}(w, z) d A_{q}(w) & =0 .
\end{aligned}
$$

Demostración. Por la Proposición 2.1.1 inciso (3) se tiene que

$$
\begin{aligned}
\int_{\mathbb{D}}\left(1-\left|\varphi_{z}(w)\right|^{2}\right)^{s} d A_{q}(w) & =\int_{\mathbb{D}} \frac{\left(1-|z|^{2}\right)^{s}\left(1-|w|^{2}\right)^{s+q}}{|1-\bar{z} w|^{2 s}} d A(w) \\
& =\left(1-|z|^{2}\right)^{s} \int_{\mathbb{D}} \frac{\left(1-|w|^{2}\right)^{s+q}}{|1-\bar{z} w|^{2 s}} d A(w) .
\end{aligned}
$$

El resultado se sigue de tomar $c=s-(q+2)$ en el Lema 1.4.2. La otra prueba es similar.

\subsection{El espacio de Bloch}

Una función analítica $f$ en $\mathbb{D}$ se dice de Bloch si satisface

$$
\|f\|_{\mathcal{B}}=\sup _{z \in \mathbb{D}}\left(1-|z|^{2}\right)\left|f^{\prime}(z)\right|<+\infty .
$$

El conjunto formado por todas las funciones de Bloch es un espacio vectorial, el cual se llamará espacio de Bloch y se denota por $\mathcal{B}$; además también se tiene que $\|f\|_{\mathcal{B}}$ es una seminorma. 
Proposición 2.2.1 La seminorma $\|f\|_{\mathcal{B}}$ es Möbius invariante, es decir $\|f \circ \varphi\|_{\mathcal{B}}=$ $\|f\|_{\mathcal{B}}$, donde $f \in \mathcal{B}$ y $\varphi$ es una transformación de Möbius de $\mathbb{D}$ en $\mathbb{D}$.

Demostración. Es suficiente probar para transformaciones de Möbius de la forma $\lambda=\varphi_{w}(z)=\frac{w-z}{1-z \bar{w}}$ con $|w|<1$. Entonces para $f \in \mathcal{B}$ se tiene por la Proposición 2.1.1 (3)

$$
\begin{aligned}
\left\|f \circ \varphi_{w}\right\|_{\mathcal{B}} & =\sup _{z \in \mathbb{D}}\left(1-|z|^{2}\right)\left|\left(f \circ \varphi_{w}\right)^{\prime}(z)\right|=\sup _{z \in \mathbb{D}}\left(1-|z|^{2}\right)\left|\varphi_{w}^{\prime}(z)\right|\left|f^{\prime}\left(\varphi_{w}(z)\right)\right| \\
& =\sup _{z \in \mathbb{D}}\left(1-\left|\varphi_{w}(z)\right|^{2}\right)\left|f^{\prime}\left(\varphi_{w}(z)\right)\right|=\sup _{\lambda \in \mathbb{D}}\left(1-|\lambda|^{2}\right)\left|f^{\prime}(\lambda)\right| \\
& =\|f\|_{\mathcal{B}}
\end{aligned}
$$

El espacio pequeño de Bloch $\mathcal{B}_{0}$, es el subespacio de funciones analíticas en $\mathbb{D}$ tales que

$$
\lim _{|z| \rightarrow 1^{-}}\left(1-|z|^{2}\right)\left|f^{\prime}(z)\right|=0 .
$$

Por continuidad el espacio $\mathcal{B}_{0}$ es subespacio de $\mathcal{B}$ y de la prueba de la Proposición 2.2.1 se tiene que $\mathcal{B}_{0}$ también es Möbius invariante.

En la siguiente proposición se dota al espacio de Bloch con cierta norma bajo la cual resulta ser un espacio de Banach.

Proposición 2.2.2 La función $\|f\|=|f(0)|+\|f\|_{\mathcal{B}}$ es una norma en $\mathcal{B}$ y el espacio de Bloch $\mathcal{B}$ es un espacio de Banach con esta norma.

Demostración. Sólo se prueba que $\mathcal{B}$ es completo. Se nota que si $g \in \mathcal{B}$ y $z \in \mathbb{D}$, entonces

$$
\begin{aligned}
|g(z)-g(0)| & =\left|z \int_{0}^{1} g^{\prime}(z t) d t\right| \leq|z| \int_{0}^{1}\left|g^{\prime}(z t)\right| d t \\
& \leq\|g\|_{\mathcal{B}} \int_{0}^{1} \frac{|z|}{1-|z|^{2} t^{2}} d t=\frac{1}{2}\|g\|_{\mathcal{B}} \log \frac{1+|z|}{1-|z|},
\end{aligned}
$$

y por tanto

$$
|g(z)-g(0)| \leq \frac{1}{2}\|f\|_{\mathcal{B}} \log \frac{1+|z|}{1-|z|} .
$$

Sea $\left\{f_{n}\right\}$ una sucesión de Cauchy en $\mathcal{B}$. Se desea probar que existe una función $f \in \mathcal{B}$ tal que, dado $\varepsilon>0$ existe $N \in \mathbb{N}$ con $\left\|f_{n}-f\right\|<\varepsilon$ para toda $n \geq N$. Puesto que $\left\{f_{n}\right\}$ 
es de Cauchy existe $N \in \mathbb{N}$ tal que $\left\|f_{n}-f_{m}\right\|<\varepsilon$ si $n \geq m>N$. Sea $0<|z|<r<1$, entonces

$$
\begin{aligned}
\left|f_{n}(z)-f_{m}(z)\right| & \leq\left|\left(f_{n}(z)-f_{m}(z)\right)-\left(f_{n}(0)-f_{m}(0)\right)\right|+\left|f_{n}(0)-f_{m}(0)\right| \\
& \leq\left\|f_{n}-f_{m}\right\|_{\mathcal{B}} \log \frac{1+|z|}{1-|z|}+\left|f_{n}(0)-f_{m}(0)\right| \\
& \leq\left\|f_{n}-f_{m}\right\|_{\mathcal{B}} \log \frac{1+|z|}{1-|z|}+\left|f_{n}(0)-f_{m}(0)\right| \log \frac{1+|z|}{1-|z|} \\
& +\left\|f_{n}-f_{m}\right\|_{\mathcal{B}}+\left|f_{n}(0)-f_{m}(0)\right| \\
& =\left\|f_{n}-f_{m}\right\|\left(1+\log \frac{1+|z|}{1-|z|}\right) \\
& <\varepsilon\left(1+\log \frac{1+|r|}{1-|r|}\right)
\end{aligned}
$$

Por lo tanto la sucesión $\left\{f_{n}\right\}$ converge uniformemente en cada subconjunto compacto de $\mathbb{D}$ y así converge uniformemente localmente a alguna función analítica $f$ en $\mathbb{D}$. Luego por el teorema de Montel, $f_{n}^{\prime}(z) \rightarrow f^{\prime}(z)$ en $\mathbb{D}$ cuando $n \rightarrow \infty$. Ahora para todo $n, m \geq N$ y $z \in \mathbb{D}$ se tiene

$$
\left(1-|z|^{2}\right)\left|f_{n}^{\prime}(z)-f_{m}^{\prime}(z)\right|+\left|f_{n}(0)-f_{m}(0)\right| \leq\left\|f_{n}-f_{m}\right\|<\frac{\varepsilon}{2} .
$$

Así, al hacer que $m$ tienda a infinito, se tiene para $n>N$,

$$
\left\|f_{n}-f\right\|_{\mathcal{B}} \leq\left\|f_{n}-f\right\|<\varepsilon .
$$

Por lo tanto $f_{n}-f \in \mathcal{B}$ y por la linealidad de $\mathcal{B}$ se tiene que $f \in \mathcal{B}$. Por lo tanto $f_{n} \rightarrow f$ en $\mathcal{B}$ y se obtiene lo deseado.

Un resultado análogo es cierto para el espacio pequeño $\mathcal{B}_{0}$.

Lema 2.2.3 El espacio $\mathcal{B}_{0}$ es completo.

Demostración. Sea $\left\{f_{n}\right\} \subset \mathcal{B}_{0}$ una sucesión de Cauchy, entonces $f_{n} \rightarrow f \in \mathcal{B}$. Debemos probar que $f \in \mathcal{B}_{0}$. Dado $\varepsilon>0$ existen $N \in \mathbb{N}$ y $\delta>0$ tales que

$$
\begin{aligned}
\left(1-|z|^{2}\right)\left|f^{\prime}(z)\right| & \leq\left(1-|z|^{2}\right)\left|f^{\prime}(z)-f_{n}^{\prime}(z)\right|+\left(1-|z|^{2}\right)\left|f_{n}^{\prime}(z)\right| \\
& \leq\left\|f(z)-f_{n}(z)\right\|_{\mathcal{B}}+\left(1-|z|^{2}\right)\left|f_{n}^{\prime}(z)\right| \\
& <\frac{\varepsilon}{2}+\frac{\varepsilon}{2}
\end{aligned}
$$

si $n \geq N, 1-\delta<|z|<1$. Así si $1-\delta<|z|<1$ se tiene que $\left(1-|z|^{2}\right)\left|f^{\prime}(z)\right|<\varepsilon$ y esto dice que $f \in \mathcal{B}_{0}$. 
Sea $C(\overline{\mathbb{D}})$ el espacio de funciones continuas en el disco unitario cerrado $\overline{\mathbb{D}}$ y $C_{0}(\mathbb{D})$ al subespacio de $C(\overline{\mathbb{D}})$ de las funciones $f$ tales que $f(z) \rightarrow 0$ cuando $|z| \rightarrow 1^{-}$. Es inmediato que $C(\overline{\mathbb{D}})$ y $C_{0}(\mathbb{D})$ son subespacios cerrados de $L^{\infty}(\mathbb{D})$.

El siguiente teorema expresa una relación entre la proyección de Bergman y los espacios de Bloch.

Teorema 2.2.4 Sea $-1<\alpha<+\infty$ y $P_{\alpha}$ la proyección de Bergman. Entonces

(1) $P_{\alpha}$ es una proyección acotada de $L^{\infty}(\mathbb{D})$ sobre $\mathcal{B}$.

(2) $P_{\alpha}$ es una proyección acotada de $C(\overline{\mathbb{D}})$ sobre $\mathcal{B}_{0}$.

(3) $P_{\alpha}$ es una proyección acotada de $C_{0}(\mathbb{D})$ sobre $\mathcal{B}_{0}$.

Las tres proyecciones anteriores son sobreyectivas.

\subsection{La Transformada de Berezin}

Aquí se define la transformada de Berezin de una función $f \in L^{1}(\mathbb{D}, d A)$ y la función $M O(f)$ asociada a ésta, si además $f \in L^{2}(\mathbb{D}, d A)$.

La Proposición 2.1.2 motiva la siguiente definición. Para cada función $f \in L^{1}(\mathbb{D}, d A)$, se define

$$
\mathbf{B} f(z)=\int_{\mathbb{D}} \frac{\left(1-|z|^{2}\right)^{2}}{|1-z \bar{w}|^{4}} f(w) d A(w), \quad z \in \mathbb{D} .
$$

$\mathrm{Al}$ operador $\mathbf{B}$ se le llama la transformada de Berezin y el hecho que sea un operador se prueba más adelante. Esta transformada tiene la particularidad que para cada función $f \in L^{1}(\mathbb{D}, d A)$ entonces $\mathbf{B} f=f$ si y sólo si $f$ es armónica, ver [HeKoZh].

De hecho hay una familia de operadores del tipo Berezin. Supóngase que $h \in$ $L^{1}\left(\mathbb{D}, d A_{\alpha}\right)$ es una función armónica acotada en $\mathbb{D}$, entonces por la propiedad del valor medio y la invarianza bajo rotación de la medida $d A_{\alpha}$, se tiene

$$
h(0)=(\alpha+1) \int_{\mathbb{D}} h(w)\left(1-|w|^{2}\right)^{\alpha} d A(w) .
$$

Reemplazando $h$ por $h \circ \varphi_{z}$ y tomando el cambio de variable

$$
\lambda=\varphi_{z}(w) \quad \text { o sea } \quad w=\varphi_{z}(\lambda)
$$


se obtiene por la Proposición 2.1.1 incisos (2) y (3)

$$
h(z)=(\alpha+1) \int_{\mathbb{D}} \frac{\left(1-|z|^{2}\right)^{\alpha+2}\left(1-|\lambda|^{2}\right)^{\alpha}}{|1-z \bar{\lambda}|^{4+2 \alpha}} h(\lambda) d A(\lambda), \quad z \in \mathbb{D} .
$$

Así para $f \in L^{1}\left(\mathbb{D}, d A_{\alpha}\right)$, se define la transformada de Berenzin $B_{\alpha}$ como

$$
\mathbf{B}_{\alpha} f(z)=(\alpha+1) \int_{\mathbb{D}} \frac{\left(1-|z|^{2}\right)^{\alpha+2}\left(1-|w|^{2}\right)^{\alpha}}{|1-z \bar{w}|^{4+2 \alpha}} f(w) d A(w), \quad z \in \mathbb{D} .
$$

Por un cambio de variable se obtiene

$$
\mathbf{B}_{\alpha} f(z)=\int_{\mathbb{D}} f \circ \varphi_{z}(w) d A_{\alpha}(w), \quad z \in \mathbb{D},
$$

para cada $f \in L^{1}\left(\mathbb{D}, d A_{\alpha}\right)$. Se observa que $\mathbf{B}_{0}=\mathbf{B}$. La siguiente proposición dice bajo que condiciones el operador $\mathbf{B}_{\alpha}$ es acotado en $L^{p}\left(\mathbb{D}, d A_{\alpha}\right)$.

Proposición 2.3.1 Supóngase que $-1<\alpha<+\infty, 1 \leq p<\infty$ y $\beta \in \mathbb{R}$. Entonces $\mathbf{B}_{\alpha}$ es acotado en $L^{p}\left(\mathbb{D}, d A_{\beta}\right)$ si y sólo si $-(\alpha+2) p<\beta+1<(\alpha+1) p$.

Demostración. De la definición del operador $\mathbf{B}_{\alpha} f$

$$
\mathbf{B}_{\alpha} f(z)=(\alpha+1)\left(1-|z|^{2}\right)^{2+\alpha} \int_{\mathbb{D}} \frac{\left(1-|w|^{2}\right)^{\alpha}}{|1-z \bar{w}|^{4+2 \alpha}} f(w) d A(w),
$$

y así aplicando el Teorema 1.4.3 se tiene que $\mathbf{B}_{\alpha} f$ es acotado si y sólo si $-(\alpha+2) p<$ $\beta+1<(\alpha+1) p$.

Se recuerda que el núcleo reproductor normalizado $k_{z}(w)$ (ver [HeKoZh]) en $A^{2}(\mathbb{D})$ es

$$
k_{z}(w)=\frac{1-|z|^{2}}{(1-\bar{z} w)^{2}}, \quad \text { con } z, w \in \mathbb{D} .
$$

Para $f \in L^{2}(\mathbb{D}, d A)$ se define

$$
M O(f)(z)=\left[B\left(|f|^{2}\right)(z)-|B f(z)|^{2}\right]^{1 / 2} .
$$

Por la desigualdad de Cauchy-Schwarz $B\left(|f|^{2}\right)(z) \geq|B f(z)|^{2}$ y así la expresión anterior está bien definida. Usando la notación de (2.7) se reescribe (2.8) de la siguiente manera. 
Proposición 2.3.2 Para $f \in L^{2}(\mathbb{D}, d A)$

$$
M O(f)(z)=\left(1-|z|^{2}\right)^{2}\left[\frac{1}{2} \int_{\mathbb{D}} \int_{\mathbb{D}}\left|\frac{f(u)-f(v)}{(1-u \bar{z})^{2}(1-v \bar{z})^{2}}\right|^{2} d A(u) d A(v)\right]^{1 / 2} .
$$

Demostración. Por la definición de la transformada de Berezin

$$
\begin{aligned}
& 2\left(B\left(|f|^{2}\right)(z)-|B f(z)|^{2}\right) \\
& =B\left(|f|^{2}\right)(z)-\int_{\mathbb{D}} f(u)\left|k_{z}(u)\right|^{2} d A(u) \int_{\mathbb{D}} \overline{f(v)}\left|k_{z}(v)\right|^{2} d A(v) \\
& -\int_{\mathbb{D}} \overline{f(u)}\left|k_{z}(u)\right|^{2} d A(u) \int_{\mathbb{D}} f(v)\left|k_{z}(v)\right|^{2} d A(v)+B\left(|f|^{2}\right)(z) \\
& =\int_{\mathbb{D}}|f(u)|^{2}\left|k_{z}(u)\right|^{2} d A(u)-\int_{\mathbb{D}} \int_{\mathbb{D}} f(u) \overline{f(v)}\left|k_{z}(u)\right|^{2}\left|k_{z}(v)\right|^{2} d A(u) d A(v) \\
& -\int_{\mathbb{D}} \int_{\mathbb{D}} \overline{f(u)} f(v)\left|k_{z}(u)\right|^{2}\left|k_{z}(v)\right|^{2} d A(u) d A(v)+\int_{\mathbb{D}}|f(v)|^{2}\left|k_{z}(v)\right|^{2} d A(v) \\
& =\int_{\mathbb{D}} \int_{\mathbb{D}}|f(u)|^{2}\left|k_{z}(u)\right|^{2}\left|k_{z}(v)\right|^{2} d A(u) d A(v) \\
& -\int_{\mathbb{D}} \int_{\mathbb{D}} f(u) \overline{f(v)}\left|k_{z}(u)\right|^{2}\left|k_{z}(v)\right|^{2} d A(u) d A(v) \\
& -\int_{\mathbb{D}} \int_{\mathbb{D}} \overline{f(u)} f(v)\left|k_{z}(u)\right|^{2}\left|k_{z}(v)\right|^{2} d A(u) d A(v) \\
& +\int_{\mathbb{D}} \int_{\mathbb{D}}|f(v)|^{2}\left|k_{z}(u)\right|^{2}\left|k_{z}(v)\right|^{2} d A(u) d A(v) \\
& =\int_{\mathbb{D}} \int_{\mathbb{D}}\left(|f(u)|^{2}-f(u) \overline{f(v)}-\overline{f(u)} f(v)+|f(v)|^{2}\right)\left|k_{z}(u)\right|^{2}\left|k_{z}(v)\right|^{2} d A(u) d A(v) \\
& =\int_{\mathbb{D}} \int_{\mathbb{D}}|f(u)-f(v)|^{2}\left|k_{z}(u)\right|^{2}\left|k_{z}(v)\right|^{2} d A(u) d A(v) .
\end{aligned}
$$

Y así se ha probado (2.9).

\subsection{Estimaciones integrales tipo Littlewood-Paley}

Los Teoremas 2.4.2 y 2.4.4 son similares a los Lemas 3.1 y 3.2 del capítulo VI en [Ga]. Para probarlos se requiere el siguiente resultado. 
Lema 2.4.1 Sea $0<r<1$ fijo. Si $\frac{r}{4} \leq x<r$ entonces

$$
\frac{16}{9} x\left(1-\frac{x^{2}}{r^{2}}\right)-x \log \frac{r}{x}>0 .
$$

Demostración. Será suficiente con probar que la función $F:\left[\frac{r}{4}, r\right] \rightarrow \mathbb{R}$ dada por

$$
F(x)=\frac{16}{9}\left(1-\frac{x^{2}}{r^{2}}\right)-\log \frac{r}{x}
$$

es no negativa. Es claro que $F\left(\frac{r}{4}\right)>0$ y $F(r)=0$. Además

$$
F^{\prime}(x)=\frac{9 r^{2}-32 x^{2}}{9 r^{2} x} \text { and } \quad F^{\prime \prime}(x)=-\frac{1}{x^{2}}-\frac{32}{9 r^{2}}<0 .
$$

Así $F(x)$ alcanza su máximo en $x=\frac{3 r}{4 \sqrt{2}} \in\left(\frac{r}{4}, r\right)$ y se puede concluir que $F(x)>0$ para todo $x \in\left[\frac{r}{4}, r\right)$.

El siguiente resultado es una desigualdad tipo Littlewood-Paley.

Teorema 2.4.2 Sean $0<r<1$ fijo y $f \in H(\mathbb{D})$. Entonces

$$
\int_{\mathbb{D}(r)}\left|f^{\prime}(z)\right|^{2} \log \frac{r}{|z|} d A(z) \leq \frac{16}{9} \int_{\mathbb{D}(r)}\left|f^{\prime}(z)\right|^{2}\left(1-\left|\frac{z}{r}\right|^{2}\right) d A(z) .
$$

Demostración. Integrando en coordenadas polares, $z=\zeta e^{i \theta}$ en $(2.10)$, se tiene que mostrar

$$
\int_{0}^{r}\left(\int_{0}^{2 \pi}\left|f^{\prime}\left(\zeta e^{i \theta}\right)\right|^{2} d \theta\right) \zeta \log \frac{r}{\zeta} d \zeta \leq \frac{16}{9} \int_{0}^{r}\left(\int_{0}^{2 \pi}\left|f^{\prime}\left(\zeta e^{i \theta}\right)\right|^{2} d \theta\right) \zeta\left(1-\frac{\zeta^{2}}{r^{2}}\right) d \zeta .
$$

Puesto que $\left|f^{\prime}(z)\right|^{2}$ es subarmónica, entonces para $0<\zeta<\zeta^{\prime}<1$

$$
h(\zeta)=\int_{0}^{2 \pi}\left|f^{\prime}\left(\zeta e^{i \theta}\right)\right|^{2} d \theta \leq \int_{0}^{2 \pi}\left|f^{\prime}\left(\zeta^{\prime} e^{i \theta}\right)\right|^{2} d \theta=h\left(\zeta^{\prime}\right) .
$$

Así, será suficiente con probar que

$$
\int_{0}^{r} h(\zeta) \zeta \log \frac{r}{\zeta} d \zeta \leq \frac{16}{9} \int_{0}^{r} h(\zeta) \zeta\left(1-\frac{\zeta^{2}}{r^{2}}\right) d \zeta .
$$

Un cálculo directo da

$$
\int_{0}^{r} \zeta \log \frac{r}{\zeta} d \zeta=\frac{16}{9} \int_{\frac{r}{2}}^{r} \zeta\left(1-\frac{\zeta^{2}}{r^{2}}\right) d \zeta
$$


Sea $\frac{r}{4} \leq t<\frac{r}{2}$. Como la función $h$ es creciente se tiene

$$
\begin{aligned}
\int_{0}^{r} h(\zeta) \zeta \log \frac{r}{\zeta} d \zeta & =\int_{0}^{t} h(\zeta) \zeta \log \frac{r}{\zeta} d \zeta+\int_{t}^{r} h(\zeta) \zeta \log \frac{r}{\zeta} d \zeta \\
& \leq h(t) \int_{0}^{t} \zeta \log \frac{r}{\zeta} d \zeta+\int_{t}^{r} h(\zeta) \zeta \log \frac{r}{\zeta} d \zeta
\end{aligned}
$$

Por (2.12) se obtiene

$$
\int_{0}^{r} \zeta \log \frac{r}{\zeta} d \zeta<\frac{16}{9} \int_{t}^{r} \zeta\left(1-\frac{\zeta^{2}}{r^{2}}\right) d \zeta
$$

por lo tanto

$$
\int_{0}^{t} \zeta \log \frac{r}{\zeta} d \zeta<\int_{t}^{r}\left(\frac{16}{9} \zeta\left(1-\frac{\zeta^{2}}{r^{2}}\right)-\zeta \log \frac{r}{\zeta}\right) d \zeta .
$$

Como $h$ es creciente, entonces por (2.13) y Lema 2.4.1 se tiene

$$
\begin{aligned}
\int_{0}^{r} h(\zeta) \zeta \log \frac{r}{\zeta} & d \zeta \\
& =\int_{0}^{t} h(\zeta) \zeta \log \frac{r}{\zeta} d \zeta+\int_{t}^{r} h(\zeta) \zeta \log \frac{r}{\zeta} d \zeta \\
& \leq h(t) \int_{t}^{r}\left(\frac{16}{9} \zeta\left(1-\frac{\zeta^{2}}{r^{2}}\right)-\zeta \log \frac{r}{\zeta}\right) d \zeta+\int_{t}^{r} h(\zeta) \zeta \log \frac{r}{\zeta} d \zeta \\
& =\int_{t}^{r} h(t)\left(\frac{16}{9} \zeta\left(1-\frac{\zeta^{2}}{r^{2}}\right)-\zeta \log \frac{r}{\zeta}\right) d \zeta+\int_{t}^{r} h(\zeta) \zeta \log \frac{r}{\zeta} d \zeta \\
& \leq \int_{t}^{r} h(\zeta)\left(\frac{16}{9} \zeta\left(1-\frac{\zeta^{2}}{r^{2}}\right)-\zeta \log \frac{r}{\zeta}\right) d \zeta+\int_{t}^{r} h(\zeta) \zeta \log \frac{r}{\zeta} d \zeta \\
& \leq \frac{16}{9} \int_{t}^{r} h(\zeta) \zeta\left(1-\frac{\zeta^{2}}{r^{2}}\right) d \zeta \\
& \leq \frac{16}{9} \int_{0}^{r} h(\zeta) \zeta\left(1-\frac{\zeta^{2}}{r^{2}}\right) d \zeta
\end{aligned}
$$

y así se tiene mostrado (2.11).

Corolario 2.4.3 Si $f \in H(\mathbb{D})$ entonces

$$
\int_{\mathbb{D}}\left|f^{\prime}(z)\right|^{2} \log \frac{1}{|z|} d A(z) \leq \frac{16}{9} \int_{\mathbb{D}}\left|f^{\prime}(z)\right|^{2}\left(1-|z|^{2}\right) d A(z) .
$$


Demostración. Por el teorema de convergencia monótona se tiene

$$
\lim _{r \rightarrow 1^{-}} \int_{\mathbb{D}(r)}\left|f^{\prime}(z)\right|^{2}\left(1-\left|\frac{z}{r}\right|^{2}\right) d A(z)=\int_{\mathbb{D}}\left|f^{\prime}(z)\right|^{2}\left(1-|z|^{2}\right) d A(z) .
$$

Sea $\left\{r_{n}\right\}$ una sucesión creciente con $\lim _{n \rightarrow \infty} r_{n}=1$ y nuevamente por convergencia monótona se obtiene

$$
\begin{aligned}
\lim _{n \rightarrow \infty} \int_{\mathbb{D}\left(r_{n}\right)}\left|f^{\prime}(z)\right|^{2} \log \frac{r_{n}}{|z|} d A(z) & =\lim _{n \rightarrow \infty} \int_{\mathbb{D}}\left|f^{\prime}(z)\right|^{2} \log \frac{r_{n}}{|z|} \chi_{\mathbb{D}\left(r_{n}\right)} d A(z) \\
& =\int_{\mathbb{D}} \lim _{n \rightarrow \infty}\left|f^{\prime}(z)\right|^{2} \log \frac{r_{n}}{|z|} \chi_{\mathbb{D}\left(r_{n}\right)} d A(z) \\
& =\int_{\mathbb{D}}\left|f^{\prime}(z)\right|^{2} \log \frac{1}{|z|} d A(z),
\end{aligned}
$$

donde $\chi$ denota la función característica. Por el Teorema 2.4.2 se obtiene el resultado.

El siguiente resultado también es una desigualdad tipo Littlewood-Paley y se usa para mostrar el Teorema 2.5.3.

Teorema 2.4.4 Para toda función holomorfa $f \in H(\mathbb{D})$

$$
\int_{\mathbb{D}}|f(z)-f(0)|^{2} d A(z) \leq \frac{16}{9} \int_{\mathbb{D}}\left(1-|z|^{2}\right)^{2}\left|f^{\prime}(z)\right|^{2} d A(z) .
$$

Demostración. Sin pérdida de generalidad se puede suponer que la integral del lado derecho es finita. Usando el teorema de Green se tiene

$$
\frac{1}{4 \pi} \int_{0}^{2 \pi}\left|f\left(r e^{i \theta}\right)-f(0)\right|^{2} d \theta=\int_{\mathbb{D}(r)}\left|f^{\prime}(z)\right|^{2} \log \frac{r}{|z|} d A(z) .
$$

Por el Teorema 2.4.2

$$
\int_{\mathbb{D}(r)}\left|f^{\prime}(z)\right|^{2} \log \frac{r}{|z|} d A(z) \leq \frac{16}{9} \int_{\mathbb{D}(r)}\left|f^{\prime}(z)\right|^{2}\left(1-\left|\frac{z}{r}\right|^{2}\right) d A(z) .
$$

Multiplicando por $r$ la desigualdad previa e integrando con respecto a $r$ se tiene

$$
\frac{1}{4 \pi} \int_{0}^{1} r d r \int_{0}^{2 \pi}\left|f\left(r e^{i \theta}\right)-f(0)\right|^{2} d \theta \leq \frac{16}{9} \int_{0}^{1} r d r \int_{\mathbb{D}(r)}\left|f^{\prime}(z)\right|^{2}\left(1-\left|\frac{z}{r}\right|^{2}\right) d A(z) .
$$


Por el teorema de Fubini se tiene

$$
\begin{aligned}
\int_{0}^{1} r d r \int_{\mathbb{D}(r)}\left|f^{\prime}(z)\right|^{2}\left(1-\left|\frac{z}{r}\right|^{2}\right) & d A(z) \\
& =\int_{\mathbb{D}} \int_{|z|}^{1}\left|f^{\prime}(z)\right|^{2} r\left(1-\left|\frac{z}{r}\right|^{2}\right) d r d A(z) \\
& =\int_{\mathbb{D}}\left|f^{\prime}(z)\right|^{2} \int_{|z|}^{1} r\left(1-\left|\frac{z}{r}\right|^{2}\right) d r d A(z) \\
& =\int_{\mathbb{D}}\left|f^{\prime}(z)\right|^{2}\left(\frac{1}{2}-\frac{|z|^{2}}{2}+\frac{1}{2}|z|^{2} \log |z|^{2}\right) d A(z) \\
& =\frac{1}{2} \int_{\mathbb{D}}\left|f^{\prime}(z)\right|^{2}\left(1-|z|^{2}+|z|^{2} \log |z|^{2}\right) d A(z) .
\end{aligned}
$$

Así (2.15) puede ser reescrita como

$$
\int_{\mathbb{D}}|f(z)-f(0)|^{2} d A(z) \leq \frac{16}{9} \int_{\mathbb{D}}\left[1-|z|^{2}+|z|^{2} \log |z|^{2}\right]\left|f^{\prime}(z)\right|^{2} d A(z) .
$$

Como $\log t \leq t-1$, para $0<t \leq 1$ entonces

$$
1-|z|^{2}+|z|^{2} \log |z|^{2} \leq\left(1-|z|^{2}\right)^{2} .
$$

Finalmente se obtiene

$$
\int_{\mathbb{D}}|f(z)-f(0)|^{2} d A(z) \leq \frac{16}{9} \int_{\mathbb{D}}\left|f^{\prime}(z)\right|^{2}\left(1-|z|^{2}\right)^{2} d A(z) .
$$

A partir del teorema anterior se tiene el siguiente resultado.

Corolario 2.4.5 Si $f \in \mathcal{B}$ entonces $f \in \mathcal{A}^{2}(\mathbb{D})$, es decir,

$$
\mathcal{B} \subset \mathcal{A}^{2}(\mathbb{D})
$$

\subsection{El espacio VMO}

Para dar una caracterización del espacio de Bloch $\mathcal{B}_{0}$ en términos del espacio $V M O$ (vanishing mean oscillation) se utilizará la métrica hiperbólica $d_{\mathbb{D}}$, también llamada métrica de Bergman la cual se define de la siguiente manera:

$$
d_{\mathbb{D}}(z, w)=\frac{1}{2} \log \frac{1+\left|\varphi_{z}(w)\right|}{1-\left|\varphi_{z}(w)\right|}, \quad \text { para cada } z, w \in \mathbb{D} .
$$


Para $z \in \mathbb{D}$ y $r>0$,

$$
D(z, r)=\left\{w \in \mathbb{D}: d_{\mathbb{D}}(z, w)<r\right\}
$$

es el disco de Bergman con centro en $z$ y radio $r$. Es bien sabido que $D(z, r)$ es un disco Euclideano con centro en $\tilde{c}$ y radio Euclidiano $\tilde{r}$ dados por

$$
\tilde{c}=\frac{\left(1-s^{2}\right) z}{1-s^{2}|z|^{2}}, \quad \tilde{r}=\frac{\left(1-|z|^{2}\right) s}{1-s^{2}|z|^{2}},
$$

donde $s=\tanh r \in(0,1)$.

Se denota por $|D(z, r)|_{A}$ al área normalizada de $D(z, r)$. Se observa que si $r>0$, entonces

$$
|D(z, r)|_{A} \approx\left(1-|z|^{2}\right)^{2} .
$$

En efecto, como $1-s^{2}|z|^{2}>1-s^{2}$, entonces tomando $C=C(s=\tanh r)=$ máx $\left\{\frac{1}{s^{2}}, \frac{s^{2}}{\left(1-s^{2}\right)^{2}}\right\}$ se obtiene

$$
\frac{1}{C}\left(1-|z|^{2}\right)^{2} \leq \frac{s^{2}\left(1-|z|^{2}\right)^{2}}{\left(1-s^{2}|z|^{2}\right)^{2}} \leq \frac{s^{2}\left(1-|z|^{2}\right)^{2}}{\left(1-s^{2}\right)^{2}} \leq C\left(1-|z|^{2}\right)^{2} .
$$

Para una función $f$, localmente integrable en $\mathbb{D}$, se define la función promedio $\widehat{f}_{r}(z)$ como

$$
\widehat{f}_{r}(z)=\frac{1}{|D(z, r)|_{A}} \int_{D(z, r)} f(w) d A(w), \quad \text { para cada } z \in \mathbb{D} .
$$

Si $f$ es también localmente cuadrado integrable con respecto a la medida de área, se define la oscilación media de $f$ en $z$ en la métrica de Bergman de la siguiente manera

$$
M O_{r}(f)(z)=\left(\frac{1}{|D(z, r)|_{A}} \int_{D(z, r)}\left|f(w)-\widehat{f}_{r}(z)\right|^{2} d A(w)\right)^{1 / 2}
$$

Se denota por $B M O_{r}(\mathbb{D})$ al espacio de las funciones en $\mathbb{D}$ con oscilación media acotada y

$$
\|f\|_{r}=\sup \left\{M O_{r}(f)(z): z \in \mathbb{D}\right\}<+\infty .
$$

El espacio $B M O_{r}(\mathbb{D})$ es también independiente del radio de Bergman $r$ y todas las seminormas $\|f\|_{r}$ son mutuamente equivalentes.

De hecho se tiene el siguiente resultado. 
Teorema 2.5.1 Sea $0<r<\infty$. Supóngase que la función $f$ es localmente cuadrado integrable en $\mathbb{D}$. Entonces $f \in B M O_{r}$ si y sólo si $f \in L^{2}(\mathbb{D}, d A)$ y la función $M O(f)$ es acotada en $\mathbb{D}$.

Sea $V M O_{r}$ el espacio de funciones $f$ localmente cuadrado integrables en $\mathbb{D}$ tales que

$$
M O_{r}(f)(z) \rightarrow 0 \quad \text { si } \quad|z| \rightarrow 1^{-} .
$$

Es claro que $V M O_{r}$ es un subespacio de $B M O_{r}$. En efecto, sea $f \in V M O_{r}$, así

$$
\lim _{|z| \rightarrow 1^{-}}\left(\frac{1}{|D(z, r)|_{A}} \int_{D(z, r)}\left|f(w)-\widehat{f}_{r}(z)\right|^{2} d A(w)\right)^{1 / 2}=0 .
$$

Así, dado $\varepsilon>0$ existe $0<R<1$ tal que si $R<|z|<1$ entonces

$$
\left(\frac{1}{|D(z, r)|_{A}} \int_{D(z, r)}\left|f(w)-\widehat{f}_{r}(z)\right|^{2} d A(w)\right)^{1 / 2}<\varepsilon .
$$

Si $z \in \mathbb{D}(R)$, por (2.16) se tiene que

$$
\frac{1}{|D(z, r)|_{A}} \approx \frac{1}{\left(1-|z|^{2}\right)^{2}} \approx \frac{1}{\left(1-R^{2}\right)^{2}}
$$

y existe un conjunto compacto $K \subset \mathbb{D}$ tal que $D(z, r) \subset K$ para cada $z \in \mathbb{D}(R)$. Así existe $C>0$ tal que

$$
\begin{aligned}
\left|\widehat{f}_{r}(z)\right| & =\left|\frac{1}{|D(z, r)|_{A}} \int_{D(z, r)} f(w) d A(w)\right| \\
& \leq \frac{C}{\left(1-R^{2}\right)^{2}} \int_{K}|f(w)| d A(w) .
\end{aligned}
$$

Por lo tanto por la desigualdad de Minkowski se tiene

$$
\begin{aligned}
& \left(\frac{1}{|D(z, r)|_{A}} \int_{D(z, r)}\left|f(w)-\widehat{f}_{r}(z)\right|^{2} d A(w)\right)^{1 / 2} \\
& \quad \leq\left(\frac{C}{\left(1-R^{2}\right)^{2}} \int_{K}\left|f(w)-\widehat{f}_{r}(z)\right|^{2} d A(w)\right)^{1 / 2} \\
& \quad \leq \frac{\sqrt{C}}{1-R^{2}}\left(\int_{K}|f(w)|^{2} d A(w)\right)^{1 / 2}+\frac{\sqrt{C}}{1-R^{2}}\left(\int_{K}\left|\widehat{f}_{r}(z)\right|^{2} d A(w)\right)^{1 / 2} .
\end{aligned}
$$

De la estimación previa se sigue que $f \in B M O_{r}$.

Se escribirá $V M O_{\partial}=V M O_{\partial}(\mathbb{D})$ para el espacio $V M O_{r}$ para cualquier $0<r<$ $+\infty . V M O_{\partial}$ es un subespacio completo de $B M O_{\partial}$ y por lo tanto es un subconjunto cerrado. Además $V M O_{\partial}$ contiene al espacio de funciones continuas en el disco unitario cerrado $\overline{\mathbb{D}}$. 
Lema 2.5.2 Sea $f \in A^{2}(\mathbb{D})$ entonces

$$
\int_{\mathbb{D}}\left|f \circ \varphi_{z}(w)-f(z)\right|^{2} d A(w)=B\left(|f|^{2}\right)(z)-|B f(z)|^{2} .
$$

Demostración. Tomando el cambio de variable $w=\varphi_{z}(\lambda)$ se obtiene

$$
\begin{aligned}
\int_{\mathbb{D}}\left|f \circ \varphi_{z}(w)-f(z)\right|^{2} d A(w) & =\int_{\mathbb{D}}\left|f \circ \varphi_{z}(w)\right|^{2} d A(w)-\int_{\mathbb{D}} f \circ \varphi_{z}(w) \overline{f(z)} d A(w) \\
& +\int_{\mathbb{D}} \overline{f \circ \varphi_{z}(w)} f(z) d A(w)+\int_{\mathbb{D}}|f(z)|^{2} d A(w) \\
& =B\left(|f|^{2}\right)(z)-(B f(z)) \overline{f(z)}-\overline{(B f(z))} f(z)+|f(z)|^{2} \\
& =B\left(|f|^{2}\right)(z)-f(z) \overline{f(z)}-\overline{f(z)} f(z)+|f(z)|^{2} \\
& =B\left(|f|^{2}\right)(z)-|f(z)|^{2} \\
& =B\left(|f|^{2}\right)(z)-|B f(z)|^{2} .
\end{aligned}
$$

Ahora se prueba el resultado principal de este capítulo.

Teorema 2.5.3 Si $f \in H(\mathbb{D})$ entonces $f \in V M O_{\partial}$ si y sólo si $f \in \mathcal{B}_{0}$, es decir

$$
V M O_{\partial} \cap H(\mathbb{D})=\mathcal{B}_{0} .
$$

Demostración. Sea $f \in \mathcal{B}_{0} \subset A^{2}(\mathbb{D})$. Por el Lema 2.5.2 y escribiendo $f \circ \varphi_{w}$ en lugar de $f$ en Lema 2.4.4 se obtiene

$$
\begin{aligned}
B\left(|f|^{2}\right)(w)-|B f(w)|^{2} & =\int_{\mathbb{D}}\left|f \circ \varphi_{w}(z)-f(w)\right|^{2} d A(z) \\
& =\int_{\mathbb{D}}\left|f \circ \varphi_{w}(z)-f \circ \varphi_{w}(0)\right|^{2} d A(z) \\
& \leq \frac{16}{9} \int_{\mathbb{D}}\left|\left(f \circ \varphi_{w}\right)^{\prime}(z)\right|^{2}\left(1-|z|^{2}\right)^{2} d A(z) \\
& =\frac{16}{9} \int_{\mathbb{D}}\left|f^{\prime}\left(\varphi_{w}(z)\right)\right|^{2}\left|\varphi_{w}^{\prime}(z)\right|^{2}\left(1-|z|^{2}\right)^{2} d A(z) \\
& =\frac{16}{9} \int_{\mathbb{D}}\left|f^{\prime}\left(\varphi_{w}(z)\right)\right|^{2} \frac{\left(1-|w|^{2}\right)^{2}\left(1-|z|^{2}\right)^{2}}{|1-\bar{z} w|^{4}} d A(z) \\
& =\frac{16}{9} \int_{\mathbb{D}}\left(1-\left|\varphi_{w}(z)\right|^{2}\right)^{2}\left|f^{\prime}\left(\varphi_{w}(z)\right)\right|^{2} d A(z) .
\end{aligned}
$$

Tomando el cambio de variable $\lambda=\varphi_{w}(z)$ se obtiene

$$
\int_{\mathbb{D}}\left(1-\left|\varphi_{w}(z)\right|^{2}\right)^{2}\left|f^{\prime}\left(\varphi_{w}(z)\right)\right|^{2} d A(z)=\int_{\mathbb{D}}\left|f^{\prime}(\lambda)\right|^{2} \frac{\left(1-|w|^{2}\right)^{2}\left(1-|\lambda|^{2}\right)^{2}}{|1-w \bar{\lambda}|^{4}} d A(\lambda)
$$


Como $f \in \mathcal{B}_{0}$ existe $R>0$ tal que

$$
\left(1-|\lambda|^{2}\right)^{2}\left|f^{\prime}(\lambda)\right|^{2}<\varepsilon, \quad \text { para todo } R<|\lambda|<1 .
$$

Así

$$
\int_{\mathbb{A}_{\mathbb{R}}}\left|f^{\prime}(\lambda)\right|^{2}\left(1-|\lambda|^{2}\right)^{2}\left|\varphi_{w}^{\prime}(\lambda)\right|^{2} d A(\lambda)<\int_{\mathbb{A}_{\mathbb{R}}} \varepsilon\left|\varphi_{w}^{\prime}(\lambda)\right|^{2} d A(\lambda)<\varepsilon .
$$

Por otra parte para $w \in \overline{B(R)}$ se obtiene $|1-\bar{\lambda} w| \geq\left(1-R^{2}\right)^{4}$ y así

$$
\frac{\left(1-|w|^{2}\right)^{2}}{\left(1-R^{2}\right)^{4}} \int_{\overline{B(R)}}\left|f^{\prime}(\lambda)\right|^{2}\left(1-|\lambda|^{2}\right)^{2} d A(\lambda) \longrightarrow 0,
$$

cuando $|w| \rightarrow 1^{-}$. Entonces

$$
B\left(|f|^{2}\right)(w)-|B f(w)|^{2} \longrightarrow 0
$$

cuando $|w| \rightarrow 1^{-}$y así

$$
f \in V M O_{\partial}(\mathbb{D}) .
$$

Se prueba el otro lado de la inclusión. Supóngase ahora que $f \in V M O_{\partial}(\mathbb{D}) \subset$ $B M O_{\partial} \subset A^{2}(\mathbb{D})$. Por la fórmula reproductora se obtiene

$$
f(z)-f(0)=\int_{\mathbb{D}} \frac{f(w)-f(0)}{(1-\bar{z} w)^{2}} d A(w) .
$$

Derivando ambos lados de la igualdad anterior

$$
f^{\prime}(z)=\int_{\mathbb{D}} \frac{2 \bar{w}(f(w)-f(0))}{(1-\bar{z} w)^{3}} d A(w) .
$$

Si $z=0$, entonces

$$
\left|f^{\prime}(0)\right|^{2} \leq 4 \int_{\mathbb{D}}|f(w)-f(0)|^{2} d A(w) .
$$

Reemplazando $f$ por $f \circ \varphi_{z}$ en la desigualdad anterior, y con $z \in \mathbb{D}$, por el Lema 2.5.2 se tiene

$$
\begin{aligned}
\left(1-|z|^{2}\right)^{2}\left|f^{\prime}(z)\right|^{2} & \leq 4 \int_{\mathbb{D}}\left|f \circ \varphi_{z}(w)-f(z)\right|^{2} d A(w) \\
& =4\left(B\left(|f|^{2}\right)(z)-|B f(z)|^{2}\right) .
\end{aligned}
$$

Así, si $f \in V M O_{\partial}$ entonces

$$
f \in \mathcal{B}_{0} .
$$

En la literatura sólo aparece la prueba de la contensión $V M O_{\delta} \cap H(\mathbb{D}) \subset \mathcal{B}_{0}$. 


\section{Capítulo 3}

\section{Espacios de Bergman Pesados $\mathcal{A}_{q}^{p}$}

En este capítulo se definen los espacios pesados de Bergman ${ }_{s} \mathcal{A}_{q}^{p}$ y $L(p, q, s)$. Se estudian algunas de sus propiedades como es la Möbius invarianza, el anidamiento de inclusiones propias y se dan caracterizaciones alternativas. Además se define una norma, para $1 \leq p<\infty$ inducida por su definición, bajo la cual los espacios son completos. Para $0<p<1$ se da una métrica que también los hara espacios completos. Destaca la prueba de la igualdad entre los espacios ${ }_{s} \mathcal{A}_{q}^{p}$ y $L(p, q, s)$ ya que la función de peso $g(w, z)$ tiene una singularidad logarítmica en $w=z$ y la función $1-\left|\varphi_{z}(w)\right|^{2}$ es continua en $\overline{\mathbb{D}}$. Por supuesto, ambas funciones se anulan en la frontera de $\mathbb{D}$.

Una motivación para la definición de los espacios $L(p, q, s)$ se obtiene de la propia definición de los espacios de Bergman al observar lo siguiente. Si $0<s<\infty$ y $z \in \mathbb{D}$ al añadir el peso $\left(1-\left|\varphi_{z}(w)\right|^{2}\right)^{s}$ en la definición del espacio de Bergman, se tiene para $f \in \mathcal{A}_{q}^{p}$

$$
\int_{\mathbb{D}}|f(w)|^{p}\left(1-\left|\varphi_{z}(w)\right|^{2}\right)^{s} d A_{q}(w)<\int_{\mathbb{D}}|f(w)|^{p} d A_{q}(w),
$$

es decir, el espacio de Bergman es un subespacio de toda una familia de espacios de Banach $L^{p}(\mathbb{D}, d \mu)$, con $d \mu=d \mu(s, z)=\left(1-\left|\varphi_{z}(w)\right|^{2}\right)^{s} d A_{q}(w)$, que se obtienen al variar los parámetros $0<s<\infty$ y $z \in \mathbb{D}$. La estimación integral anterior permite considerar una familia restringida a un parámetro, a saber el conjunto de funciones analíticas $f: \mathbb{D} \rightarrow \mathbb{C}$ que satisfacen

$$
\sup _{z \in \mathbb{D}} \int_{\mathbb{D}}|f(w)|^{p}\left(1-\left|\varphi_{z}(w)\right|^{2}\right)^{s} d A_{q}(w) \leq \int_{\mathbb{D}}|f(w)|^{p} d A_{q}(w),
$$


para $0<s<1$. Luego se tiene

$$
\mathcal{A}_{q}^{p} \subset L(p, q, s) \subset \bigcap_{z \in \mathbb{D}} L^{p}(\mathbb{D}, d \mu(s, z))
$$

Así se puede considerar cada elemento $L(p, q, s)$, de esta familia, una generalización natural de los espacios de Bergman y en particular se probará que son una sucesión anidada de espacios de Banach con respecto al parámetro $s$.

\subsection{Espacios ${ }_{s} \mathcal{A}_{q}^{p}$}

Se recuerda que para $z, w \in \mathbb{D}$, la función de Green del disco unitario $\mathbb{D}$, está dada por

$$
g(w, z)=\ln \frac{|1-\bar{z} w|}{|z-w|}=\ln \frac{1}{\left|\varphi_{z}(w)\right|} .
$$

Sea $\mathcal{H}$ el espacio de funciones analíticas $f: \mathbb{D} \rightarrow \mathbb{C}$. Para $0<p<\infty,-2<q<\infty$, $0 \leq s<\infty$ y $f \in \mathcal{H}$ se define

$$
h_{p, q, s}(f)(z):=\int_{\mathbb{D}}|f(w)|^{p} g^{s}(w, z) d A_{q}(w),
$$

donde $d A_{q}(w):=\left(1-|w|^{2}\right)^{q} d A(w)$. El espacio $p$-Bergman $q$, s-pesado está dado por

$$
{ }_{s} \mathcal{A}_{q}^{p}:=\left\{f \in \mathcal{H}: \sup _{z \in \mathbb{D}} h_{p, q, s}(f)(z)<\infty\right\}
$$

y para $0<s<\infty$, el espacio pequeño $p$-Bergman $q$, $s$-pesado es

$$
{ }_{s, 0} \mathcal{A}_{q}^{p}:=\left\{f \in \mathcal{H}: \lim _{|z| \rightarrow 1^{-1}} h_{p, q, s}(f)(z)=0\right\} .
$$

Se observa que la medida $d A_{q}$ no está normalizada.

De la misma forma, para $0<p<\infty,-2<q<\infty, 0 \leq s<\infty$ y $f \in \mathcal{H}$ se define

$$
\begin{gathered}
l_{p, q, s}(f)(z):=\int_{\mathbb{D}}|f(w)|^{p}\left(1-\left|\varphi_{z}(w)\right|^{2}\right)^{s} d A_{q}(w), \\
L(p, q, s):=\left\{f \in \mathcal{H}: \sup _{z \in \mathbb{D}} l_{p, q, s}(f)(z)<\infty\right\}
\end{gathered}
$$

y para $0<s<\infty$ el espacio pequeño correspondiente es

$$
L_{0}(p, q, s):=\left\{f \in \mathcal{H}: \lim _{|z| \rightarrow 1^{-}} l_{p, q, s}(f)(z)=0\right\} .
$$


Se escribe $L_{p}=L(p, 0,0)$ y se observa que ${ }_{0} \mathcal{A}_{q}^{p}=L(p, q, 0)=\mathcal{A}_{q}^{p}$ son los espacios clásicos de Bergman pesados de funciones analíticas, ver [HeKoZh]. Si $f \in{ }_{s} \mathcal{A}_{q}^{p}$ ó $f \in L(p, q, s)$ se escribe

$$
\|f\|_{g}=\|f\|_{g, p, q, s}=\sup _{z \in \mathbb{D}}\left(h_{p, q, s}(f)(z)\right)^{\frac{1}{p}}
$$

y

$$
\|f\|_{\varphi}=\|f\|_{\varphi, p, q, s}=\sup _{z \in \mathbb{D}}\left(l_{p, q, s}(f)(z)\right)^{\frac{1}{p}} .
$$

Sea $0<\alpha<\infty$. Se dice que $f \in \mathcal{H}$ pertenece al espacio $\alpha$-growth (ó $\alpha$-tipo espacio de Bloch), denotado por $\mathcal{A}^{-\alpha}$, si

$$
\|f\|_{-\alpha}=\sup _{z \in \mathbb{D}}\left(1-|z|^{2}\right)^{\alpha}|f(z)|<\infty
$$

y pertenece al espacio pequeño $\alpha$-growth, denotado por $\mathcal{A}^{-\alpha, 0}$, si

$$
\|f\|_{-\alpha}=\lim _{|z| \rightarrow 1^{-}}\left(1-|z|^{2}\right)^{\alpha}|f(z)|=0 .
$$

Es claro que $\mathcal{A}^{-\alpha, 0} \subset \mathcal{A}^{-\alpha}$ y estos espacios son completos (ver [HeKoZh], sección 4.3).

Para mostrar el siguiente teorema, se necesitará el siguiente resultado.

Lema 3.1.1 Sean $0 \leq k_{1}<\infty, 0 \leq k_{2}<\infty, y k_{1}-k_{2}>-1$, entonces

$$
C\left(k_{1}, k_{2}\right)=\int_{\mathbb{D}}\left(\log \frac{1}{|z|}\right)^{k_{1}}\left(1-|z|^{2}\right)^{-k_{2}} d A(z)<\infty .
$$

Demostración. Sea $D_{\frac{1}{4}}:=\left\{z \in \mathbb{D}:|z|<\frac{1}{4}\right\}$. De esto es obvio que $1-\left(\frac{1}{4}\right) \leq 1-|z|^{2}$, es decir, $\frac{15}{16} \leq 1-|z|^{2}$ para toda $z \in D_{\frac{1}{4}}$. Así se tiene que

$$
\begin{aligned}
\int_{D_{\frac{1}{4}}}\left(\log \frac{1}{|z|}\right)^{k_{1}}\left(1-|z|^{2}\right)^{-k_{2}} d A(z) & \leq\left(\frac{16}{15}\right)^{k_{2}} \int_{D_{\frac{1}{4}}}\left(\log \frac{1}{|z|}\right)^{k_{1}} d A(z) \quad(3.4) \\
& \leq\left(\frac{16}{15}\right)^{k_{2}} \int_{0}^{2 \pi} \int_{0}^{1}\left(\log \frac{1}{r}\right)^{k_{1}} r d r d \theta \\
& =2 \pi\left(\frac{16}{15}\right)^{k_{2}} \int_{0}^{\infty} t^{k_{1}} e^{-2 t} d t \\
& =\left(\frac{16}{15}\right)^{k_{2}} \frac{\pi \Gamma\left(k_{2}+1\right)}{2^{k_{1}}}<\infty
\end{aligned}
$$


Por otro lado, se tiene que $\log \frac{1}{|z|} \leq 4\left(1-|z|^{2}\right)$ para toda $z \in \mathbb{D} \backslash D_{\frac{1}{4}}$, por lo cual

$$
\begin{aligned}
\int_{\mathbb{D} \backslash D_{\frac{1}{4}}}\left(\log \frac{1}{|z|}\right)^{k_{1}}\left(1-|z|^{2}\right)^{-k_{2}} d A(z) & \leq 4^{k_{1}} \int_{\mathbb{D} \backslash D_{\frac{1}{4}}}\left(1-|z|^{2}\right)^{k_{1}-k_{2}} d A(z)(3.5) \\
& =4^{k_{1}} \int_{0}^{2 \pi} \int_{0}^{1}\left(1-r^{2}\right)^{k_{1}-k_{2}} r d r d \theta \\
& =\frac{4^{k_{1}} \pi}{1+k_{1}-k_{2}}<\infty
\end{aligned}
$$

Los espacios $\alpha$-growth tienen la siguiente caracterización.

Teorema 3.1.2 Sea $0<p<\infty, 0 \leq \alpha<\infty, 0<r<1$ y $1<s<\infty$. Entonces una de las siguientes cantidades es finita si y sólo si las otras también lo son:

(i) $\sup _{z \in \mathbb{D}}\left(1-|z|^{2}\right)^{p \alpha}|f(z)|^{p}$;

(ii) $\sup _{z \in \mathbb{D}} \frac{1}{|D(z, r)|^{1-\frac{p \alpha}{2}}} \int_{D(z, r)}|f(w)|^{p} d A(w)$;

(iii) $\sup _{z \in \mathbb{D}} \int_{D(z, r)}|f(w)|^{p} d A_{p \alpha-2}(w)$;

(iv) $\sup _{z \in \mathbb{D}} \int_{\mathbb{D}}|f(w)|^{p}\left(1-\left|\varphi_{z}(w)\right|^{2}\right)^{s} d A_{p \alpha-2}(w) ;$

(v) $\sup _{z \in \mathbb{D}} \int_{\mathbb{D}}|f(w)|^{p} g^{s}(w, z) d A_{p \alpha-2}(w) ;$

(vi) $\sup _{z \in \mathbb{D}} \int_{\mathbb{D}}|f(w)|^{p}\left(\log \frac{1}{|w|}\right)^{p \alpha}\left|\varphi_{z}^{\prime}(w)\right|^{2} d A(w)$.

Demostración. La subarmonicidad de $|f(z)|^{p}$ para $0<p<\infty$ es la clave para la prueba y esta es similar a la prueba del Teorema 1 de R. Zhao en [Zha].

Un resultado análogo es cierto con el espacio $\alpha$-growth pequeño, (ver Teorema 2 de R. Zhao en [Zha]).

El siguiente teorema da una relación estrecha entre los espacios ${ }_{s} \mathcal{A}_{q}^{p}$ y los espacios $\mathcal{A}^{-s}$.

Teorema 3.1.3 Sean $0<p<\infty y-2<q<\infty$. Si $0<s<\infty$, entonces ${ }_{s} \mathcal{A}_{q}^{p} \subset$ $\mathcal{A}^{-\frac{q+2}{p}} y_{s, 0} \mathcal{A}_{q}^{p} \subset \mathcal{A}_{0}^{-\frac{q+2}{p}}$. Si $1<s<\infty$, entonces ${ }_{s} \mathcal{A}_{q}^{p}=\mathcal{A}^{-\frac{q+2}{p}} y_{s, 0} \mathcal{A}_{q}^{p}=\mathcal{A}_{0}^{-\frac{q+2}{p}}$. 
Demostración. Sean $f \in{ }_{s} \mathcal{A}_{q}^{p}$ y $0<R<1$ fijos. Entonces por la definición de $D(z, R)$, el cambio de variable $w=\varphi_{z}(\zeta)$ y subarmonicidad se tiene

$$
\begin{aligned}
\int_{\mathbb{D}}|f(w)|^{p} g^{s}(w, z) & d A_{q}(w) \\
& \geq \int_{D(z, R)}|f(w)|^{p} g^{s}(w, z) d A_{q}(w) \\
& \geq \ln ^{s} \frac{1}{R} \int_{\mathbb{D}_{R}}\left|f\left(\varphi_{z}(\zeta)\right)\right|^{p}\left(1-\left|\varphi_{z}(\zeta)\right|^{2}\right)^{q}\left|\varphi^{\prime}(\zeta)\right|^{2} d A(\zeta) \\
& \geq \frac{1}{2^{2 q+4} \pi} \ln ^{s} \frac{1}{R}\left(1-|z|^{2}\right)^{q+2} \int_{0}^{R}\left(1-\rho^{2}\right)^{q} \rho \int_{0}^{2 \pi}\left|f\left(\varphi_{z}\left(\rho e^{i \theta}\right)\right)\right|^{p} d \theta d \rho \\
& \geq \frac{1}{2^{2 q+4} \pi} \ln ^{s} \frac{1}{R}\left(1-|z|^{2}\right)^{q+2}|f(z)|^{p} \int_{0}^{R}\left(1-\rho^{2}\right)^{q} \rho d \rho \\
& =C(R)\left(1-|z|^{2}\right)^{q+2}|f(z)|^{p} .
\end{aligned}
$$

Las primeras dos afirmaciones se siguen de la estimación previa.

Si $f \in \mathcal{A}^{-\frac{q+2}{p}}$, de nuevo mediante el cambio de variable $w=\varphi_{z}(\zeta)$ se tiene que

$$
\begin{aligned}
\int_{\mathbb{D}}|f(w)|^{p} g^{s}(w, z) d A_{q}(w) & \leq\|f\|_{\frac{q+2}{p}}^{p} \int_{\mathbb{D}} \frac{1}{\left(1-|w|^{2}\right)^{2}} g^{s}(w, z) d A(w) \\
& =\|f\|_{\frac{q+2}{p}}^{p} \int_{\mathbb{D}} \frac{1}{\left(1-\left|\varphi_{z}(\zeta)\right|^{2}\right)^{2}} \ln ^{s} \frac{1}{|\zeta|}\left|\varphi_{z}^{\prime}(\zeta)\right|^{2} d A(\zeta) \\
& =\|f\|_{\frac{q+2}{p}}^{p} \int_{\mathbb{D}} \frac{1}{\left(1-|\zeta|^{2}\right)^{2}} \ln ^{s} \frac{1}{|\zeta|} d A(\zeta) .
\end{aligned}
$$

Entonces para $1<s<\infty$, la última integral es finita pues

$$
\lim _{r \rightarrow 0^{+}} r \ln ^{s} \frac{1}{r}=0 \quad \text { y } \quad \frac{\ln ^{s} \frac{1}{r}}{\left(1-r^{2}\right)^{2}} \approx(1-r)^{s-2} \text { cuando } r \rightarrow 1^{-} .
$$

Sea $f \in \mathcal{A}_{0}^{-\frac{q+2}{p}}$. Dado $\varepsilon>0$, existe $0<R<1$ tal que

$$
|f(w)|^{p} \leq \frac{\varepsilon}{\left(1-|w|^{2}\right)^{q+2}} \quad \text { para toda } w \in \mathbb{A}_{R}
$$


Así

$$
\begin{aligned}
\int_{\mathbb{A}_{R}}|f(w)|^{p} g^{s}(w, z) d A_{q}(w) & \leq \varepsilon \int_{\mathbb{A}_{R}} \frac{g^{s}(w, z)}{\left(1-|w|^{2}\right)^{2}} d A(w) \\
& \leq \varepsilon \int_{\mathbb{A}_{R}} \frac{\left(1-\left|\varphi_{z}(w)\right|^{2}\right)^{s}}{\left(1-|w|^{2}\right)^{2}} d A(w) \\
& \leq \varepsilon\left(1-|z|^{2}\right)^{s} \int_{\mathbb{D}} \frac{\left(1-|w|^{2}\right)^{s-2}}{|1-z \bar{w}|^{2 s}} d A(w) \\
& <\varepsilon .
\end{aligned}
$$

La última desigualdad se obtiene al aplicar el Teorema 1.4.2 tomado $c=s>0$. Además

$$
\int_{\mathbb{D}_{R}}|f(w)|^{p} g^{s}(w, z) d A_{q}(w) \leq \operatorname{máx}_{w \in \overline{\mathbb{D}}_{R}}|f(w)|^{p} \int_{\mathbb{D}_{R}} g^{s}(w, z) d A_{q}(w)
$$

y se concluye la prueba aplicando el Lema 2.1.5 .

Corolario 3.1.4 Sean $0<p<\infty y-2<q<\infty$. Entonces $\mathcal{A}_{q}^{p} \subset \mathcal{A}_{0}^{-\frac{q+2}{p}} y\|\|_{\frac{q+2}{p}} \leq$ $C\|\|_{g}$.

Demostración. Por (2.3), $|D(z, R)| \rightarrow 0$ cuando $|z| \rightarrow 1^{-}$y se tiene el resultado aplicando la continuidad absoluta de la integral en la prueba del Teorema 3.1.3.

Algunas propiedades inmediatas de los espacios ${ }_{s} \mathcal{A}_{q}^{p}$ y $L(p, q, s)$ son las siguientes. Sean $0<p<\infty$ y $0<s<s^{\prime}<\infty$, luego

$$
L(p, q, s) \subset L\left(p, q, s^{\prime}\right) \quad \text { y } \quad L_{0}(p, q, s) \subset L_{0}\left(p, q, s^{\prime}\right) ;
$$

y puesto que $1-x^{2} \leq 2 \ln \frac{1}{x}$ para toda $x \in(0,1]$ entonces

$$
{ }_{s} \mathcal{A}_{q}^{p} \subset L(p, q, s) \quad \mathrm{y} \quad{ }_{s, 0} \mathcal{A}_{q}^{p} \subset L_{0}(p, q, s)
$$

Teorema 3.1.5 Sean $0<p<\infty,-2<q<\infty$ y $0<s<\infty$, con $-1<q+s$. Si $f, g \in L(p, q, s)$ (ó $\left.L_{0}(p, q, s)\right)$, entonces $\lambda f+\eta g \in L(p, q, s)\left(o ́ ~ L_{0}(p, q, s)\right)$ para $\lambda, \eta \in \mathbb{C}$. Además, si $0<p<r<\infty$, entonces $L(r, q, s) \subset L_{0}(p, q, s)$.

Demostración. Se define para cada conjunto medible $E \subset \mathbb{D}$,

$$
\nu_{z, q, s}(E)=\int_{E}\left(1-\left|\varphi_{z}(w)\right|^{2}\right)^{s} d A_{q}(w) .
$$


Así $\nu_{z, q, s}(\mathbb{D})<\infty$ por el Lema 1.4.2 y por el Lema 2.1.5 se tiene que

$$
\lim _{|z| \rightarrow 1^{-}} \nu_{z, q, s}(\mathbb{D})=0 \text {. }
$$

Además $\nu_{z, q, s}$ es absolutamente continua con respecto a la medida de Lebesgue en $\mathbb{D}$ $\mathrm{y}$

$$
\int_{\mathbb{D}}|f(w)|^{p} d \nu_{z, q, s}(w)=\int_{\mathbb{D}}|f(w)|^{p}\left(1-\left|\varphi_{z}(w)\right|^{2}\right)^{s} d A_{q}(w) .
$$

Sean $f, g \in L(p, q, s)$. El resultado se tiene de la siguiente estimación.

$$
|\lambda f(w)+\eta g(w)|^{p} \leq 2^{p}\left(|\lambda|^{p}|f(w)|^{p}+|\eta|^{p}|g(w)|^{p}\right)
$$

y la desigualdad de Hölder (ver [HeSt], Teorema 13.17)

$$
\left(\int_{\mathbb{D}}|f(w)|^{p} d \nu_{z, q, s}(w)\right)^{\frac{1}{p}} \leq\left(\int_{\mathbb{D}}|f(w)|^{r} d \nu_{z, q, s}(w)\right)^{\frac{1}{r}} \nu_{z, q, s}(\mathbb{D})^{\frac{1}{p}-\frac{1}{r}}
$$

Teorema 3.1.6 Sean $1 \leq p<\infty,-2<q<\infty$ y $0<s<\infty$. Entonces \|\|$_{g} y$ \|\|$_{\varphi}$ definen normas en ${ }_{s} \mathcal{A}_{q}^{p}$ y $L(p, q, s)$, respectivamente. Con estas normas ${ }_{s} \mathcal{A}_{q}^{p}$ y $L(p, q, s)$ son espacios de Banach.

Demostración. Es inmediato que $\|\cdot\|_{g} \mathrm{y}\|\cdot\|_{\varphi}$ son normas. Se probará sólo que ${ }_{s} \mathcal{A}_{q}^{p}$ es completo. Sea $\left\{f_{n}\right\}$ una sucesión de Cauchy en ${ }_{s} \mathcal{A}_{q}^{p}$. Por el Corolario 3.1.4, $\left\{f_{n}\right\}$ es también una sucesión de Cauchy en $\mathcal{A}^{-\frac{q+2}{p}}$. Entonces $\left\{f_{n}\right\}$ converge uniformemente en subconjuntos compactos a $f \in \mathcal{A}^{-\frac{q+2}{p}}$. Dado $\epsilon>0$, existe $N>0$ tal que si $n \geq m \geq N$ se tiene

$$
\int_{\mathbb{D}}\left|f_{n}(w)-f_{m}(w)\right|^{p} g^{s}(w, z) d A_{q}(w) \leq\left\|f_{n}-f_{m}\right\|_{g}^{p}<\left(\frac{\epsilon}{2}\right)^{p} .
$$

Por el Lema de Fatou

$$
\begin{aligned}
\int_{\mathbb{D}}\left|f(w)-f_{m}(w)\right|^{p} g^{s}(w, z) d A_{q}(w) & \leq \liminf _{n \rightarrow \infty} \int_{\mathbb{D}}\left|f_{n}(w)-f_{m}(w)\right|^{p} g^{s}(w, z) d A_{q}(w) \\
& \leq\left(\frac{\epsilon}{2}\right)^{p} .
\end{aligned}
$$

Tomando el supremo con respecto a $z \in \mathbb{D}$ se ve que $f-f_{m} \in{ }_{s} \mathcal{A}_{q}^{p}$, por lo tanto $f=\left(f-f_{m}\right)+f_{m} \in{ }_{s} \mathcal{A}_{q}^{p}$. Además $\left\|f-f_{m}\right\|_{g} \leq \frac{\epsilon}{2}$ si $m \geq N$.

El siguiente resultado dice que $L(p, q, s){ }_{0}{ }_{s} \mathcal{A}_{q}^{p}$ pueden ser triviales. 
Proposición 3.1.7 Sea $0<p<\infty,-2<q<\infty$ y $0<s<\infty$ con $q+s \leq-1$. Entonces el espacio $L(p, q, s)={ }_{s} \mathcal{A}_{q}^{p}$ y consiste sólo de la función constante 0 .

Demostración. Sea $f \in L(p, q, s)$ una función distinta de cero. Sea $0<b<1$ fijo. Puesto que $|f(z)|^{p}$ es una función subarmónica,

$$
\begin{aligned}
l_{p, q, s}(f)(0) & =\int_{\mathbb{D}}|f(w)|^{p}\left(1-|w|^{2}\right)^{s} d A_{q}(w) \geq \frac{1}{\pi} \int_{b}^{1} \int_{0}^{2 \pi}\left|f\left(r e^{i \theta}\right)\right|^{p}\left(1-r^{2}\right)^{q+s} r d \theta d r \\
& \geq \frac{1}{\pi} \int_{0}^{2 \pi}\left|f\left(b e^{i \theta}\right)\right|^{p} d \theta \int_{b}^{1}\left(1-r^{2}\right)^{q+s} r d r=\infty .
\end{aligned}
$$

Así se obtiene una contradición. La otra prueba es similar.

Se define

$$
\mathcal{H}^{\infty}=\{f \in \mathcal{H}: f \text { es acotada }\} .
$$

Los espacios ${ }_{s, 0} \mathcal{A}_{q}^{p}$ son no vacíos, ya que por el Lema 2.1.5

$$
\mathcal{H}^{\infty} \subset \bigcap\left\{{ }_{s, 0} \mathcal{A}_{q}^{p}: 0<p<\infty,-2<q<\infty, 0<s<\infty,-1<q+s .\right\} .
$$

De esta forma se puede mejorar el Corolario 3.1.4

Teorema 3.1.8 Sean $0<p<\infty y-2<q<\infty$. Entonces

$$
\mathcal{A}_{q}^{p} \subset \bigcap_{0<s<\infty} s, 0 \mathcal{A}_{q}^{p} \quad y \quad \mathcal{A}_{q}^{p} \subset \bigcap_{0<s<\infty}{ }_{0,0} L_{q}^{p}
$$

Demostración. Se probará la primera inclusión. La segunda se hace de manera similar. Sean $f \in \mathcal{A}_{q}^{p}, 1 \leq s<\infty$ y $\varepsilon>0$. Por el Corolario 3.1.4, existe $0<R<1$ tal que

$$
\left(1-|w|^{2}\right)^{q+2}|f(w)|^{p}<\varepsilon \quad \text { para toda } \quad w \in \mathbb{A}_{R}
$$

y

$$
\int_{\mathbb{A}_{R}}|f(w)|^{p} d A_{q}(w)<\varepsilon
$$

por la continuidad absoluta de la integral. Se considera la descomposición

$$
h_{p, q, s}(f)(z)=\int_{\mathbb{D}_{R}}|f(w)|^{p} g^{s}(w, z) d A_{q}(w)+\int_{\mathbb{A}_{R}}|f(w)|^{p} g^{s}(w, z) d A_{q}(w) .
$$

Por el Lema 2.1.5 la primera integral tiende a 0 cuando $|z| \rightarrow 1^{-}$. Para estimar el segundo sumando integral se subdividirá de la siguiente forma. Por Proposición 2.1.4 
se puede elegir $R^{\prime}$ fijo, tal que $\frac{1}{e}<R^{\prime}<1$ tal que $D\left(z, R^{\prime}\right) \subset \mathbb{A}_{R}$, así:

$$
\begin{aligned}
& \int_{\mathbb{A}_{R}}|f(w)|^{p} g^{s}(w, z) d A_{q}(w) \\
&=\int_{\mathbb{A}_{R} \backslash D\left(z, R^{\prime}\right)}|f(w)|^{p} g^{s}(w, z) d A_{q}(w)+\int_{D\left(z, R^{\prime}\right)}|f(w)|^{p} g^{s}(w, z) d A_{q}(w) .
\end{aligned}
$$

Ahora por (3.8) se tiene que

$$
\int_{\mathbb{A}_{R} \backslash D\left(z, R^{\prime}\right)}|f(w)|^{p} g^{s}(w, z) d A_{q}(w) \leq \ln ^{s} \frac{1}{R^{\prime}} \int_{\mathbb{A}_{R} \backslash D\left(z, R^{\prime}\right)}|f(w)|^{p} d A_{q}(w)<\varepsilon \ln ^{s} \frac{1}{R^{\prime}}<\varepsilon .
$$

Por otra parte, de (3.7) y el cambio de variable $w=\varphi_{z}(\zeta)$ se tiene

$$
\begin{aligned}
\int_{D\left(z, R^{\prime}\right)}|f(w)|^{p} g^{s}(w, z) d A_{q}(w) & \leq \int_{D\left(z, R^{\prime}\right)} \frac{\varepsilon}{\left(1-|w|^{2}\right)^{q+2}} g^{s}(w, z) d A_{q}(w) \\
& =\varepsilon \int_{\mathbb{D}_{R^{\prime}}} \frac{1}{\left(1-\left|\varphi_{z}(\zeta)\right|^{2}\right)^{2}} \ln ^{s} \frac{1}{|\zeta|}\left|\varphi^{\prime}(\zeta)\right|^{2} d A(\zeta) \\
& \leq \varepsilon \int_{\mathbb{D}_{R^{\prime}}} \frac{1}{\left(1-|\zeta|^{2}\right)^{2}} \ln ^{s} \frac{1}{|\zeta|} d A(\zeta)
\end{aligned}
$$

Si $1<s<\infty$, entonces por el Lema 3.1.1 se tiene que

$$
\int_{\mathbb{D}_{R^{\prime}}} \frac{1}{\left(1-|\zeta|^{2}\right)^{2}} \ln ^{s} \frac{1}{|\zeta|} d A(\zeta) \leq \int_{\mathbb{D}} \frac{1}{\left(1-|\zeta|^{2}\right)^{2}} \ln ^{s} \frac{1}{|\zeta|} d A(\zeta)<\infty
$$

Si $s=1$ se tiene que

$$
\begin{aligned}
\int_{\mathbb{D}_{R^{\prime}}} \frac{1}{\left(1-|\zeta|^{2}\right)^{2}} \ln \frac{1}{|\zeta|} d A(\zeta) & =\frac{1}{\pi} \int_{0}^{2 \pi} \int_{0}^{R^{\prime}} \frac{1}{\left(1-r^{2}\right)^{2}} \ln \frac{1}{r} r d r d \theta \\
& \leq \frac{2}{\left(1-R^{\prime}\right)^{2}} \int_{0}^{R^{\prime}} \ln \frac{1}{r} r d r<\infty
\end{aligned}
$$

Por lo tanto

$$
\int_{D\left(z, R^{\prime}\right)}|f(w)|^{p} g^{s}(w, z) d A_{q}(w)<\varepsilon C\left(R^{\prime}\right)
$$

para toda $1 \leq s<\infty$

Por otro lado, sea $0<s<1$ con $s+s^{\prime}=1$. Por la desigualdad de Hölder se tiene 
que

$$
\begin{aligned}
\int_{\mathbb{D}}|f(w)|^{p} & g^{s}(w, z) d A_{q}(w) \\
& =\int_{\mathbb{D}}|f(w)|^{p s} g^{s}(w, z)|f(w)|^{p-p s} d A_{q}(w) \\
& \leq\left[\int_{\mathbb{D}}\left(|f(w)|^{p s} g^{s}(w, z)\right)^{\frac{1}{s}} d A_{q}(w)\right]^{s}\left[\int_{\mathbb{D}}\left(|f(w)|^{p-p s}\right)^{\frac{1}{1-s}} d A_{q}(w)\right]^{1-s} \\
& =\left[\int_{\mathbb{D}}|f(w)|^{p} g(w, z) d A_{q}(w)\right]^{s}\left[\int_{\mathbb{D}}|f(w)|^{p} d A_{q}(w)\right]^{1-s},
\end{aligned}
$$

y así del hecho que $\mathcal{A}_{q}^{p} \subset_{1,0} \mathcal{A}_{q}^{p}$ se tiene que

$$
\lim _{|z| \rightarrow 1^{-}} \int_{\mathbb{D}}|f(w)|^{p} g^{s}(w, z) d A_{q}(w)=0,
$$

para toda $0<s<1$. Por lo tanto $f \in{ }_{s, 0} \mathcal{A}_{q}^{p}$ para todo $0<s<\infty$ y esto concluye la prueba.

Los espacios ${ }_{s} \mathcal{A}_{q}^{p}$ y $L(p, q, s)$ son Möbius invariantes en el siguiente sentido:

Proposición 3.1.9 Sean $z \in \mathbb{D}, 0<p<\infty,-2<q<\infty$ y $0 \leq s<\infty$. Si $f \in{ }_{s} \mathcal{A}_{q}^{p}$ (ó $f \in L(p, q, s)$ ) entonces $f \circ \varphi_{z} \in{ }_{s} \mathcal{A}_{q}^{p}$ (or $f \circ \varphi_{z} \in L(p, q, s)$ ), es decir, se necesita probar para toda $\varphi$ de Möbius y no sólo para $\varphi_{z}$,

$$
\sup _{b \in \mathbb{D}} \int_{\mathbb{D}}\left|f\left(\varphi_{z}(w)\right)\right|^{p} g^{s}(w, b) d A_{q}(w)<\infty \text {. }
$$

Demostración. Sea $z \in \mathbb{D}$ fijo. Se denota por $w=\varphi_{z}(v), b=\varphi_{z}(c)$. Como la función de Green es conformemente invariante, $g\left(\varphi_{z}(v), \varphi_{z}(c)\right)=g(v, c)$. Entonces por $(2.1)$ $\mathrm{y}(2.2)$

$$
\begin{aligned}
\int_{\mathbb{D}}\left|f\left(\varphi_{z}(w)\right)\right|^{p} & g^{s}(w, b) d A_{q}(w) \\
& =\int_{\mathbb{D}}\left|f\left(\varphi_{z}\left(\varphi_{z}(v)\right)\right)\right|^{p}\left|\varphi_{z}^{\prime}(v)\right|^{2}\left(1-\left|\varphi_{z}(v)\right|^{2}\right)^{q} g^{s}\left(\varphi_{z}(v), \varphi_{z}(c)\right) d A(v) \\
& \leq \rho(z,|q|+2) \int_{\mathbb{D}}|f(v)|^{p}\left(1-|v|^{2}\right)^{q} g^{s}(v, c) d A(v) \\
& =\rho(z,|q|+2) \int_{\mathbb{D}}|f(v)|^{p} g^{s}(v, c) d A_{q}(v) .
\end{aligned}
$$


Tomando el supremo se obtiene el resultado.

Se observa que el resultado previo también es válido para los espacios pequeños, pues si $b=\varphi_{z}(c)$ entonces $|b| \rightarrow 1$ cuando $|c| \rightarrow 1$.

Se necesitará el siguiente resultado.

Lema 3.1.10 Sean $0<p<\infty,-2<q<\infty, 0 \leq s<\infty$ y $z \in \mathbb{D}$. Entonces

$$
\frac{1}{\rho(z,|q|+2)} l_{p, q, s}(f)(z) \leq l_{p, q, s}\left(f \circ \varphi_{z}\right)(0) \leq \rho(z,|q|+2) l_{p, q, s}(f)(z) .
$$

Demostración. Supóngase que $l_{p, q, s}(f)(z)<\infty$. Entonces por la fórmula de cambio de variable, tomando $v=\varphi_{z}(w)$, se obtiene

$$
\begin{array}{rl}
\int_{\mathbb{D}}\left|f\left(\varphi_{z}(v)\right)\right|^{p}\left(1-|v|^{2}\right)^{q+s} & d A(v) \\
& =\int_{\mathbb{D}}|f(w)|^{p}\left|\varphi_{z}^{\prime}(w)\right|^{2}\left(1-\left|\varphi_{z}(w)\right|^{2}\right)^{q+s} d A(w) \\
& \leq \rho(z,|q|+2) \int_{\mathbb{D}}|f(w)|^{p}\left(1-|w|^{2}\right)^{q}\left(1-\left|\varphi_{z}(w)\right|^{2}\right)^{s} d A(w)
\end{array}
$$

A la inversa, si $l_{p, q, s}\left(f \circ \varphi_{z}\right)(0)<\infty$, entonces con $v=\varphi_{z}(w)$ :

$$
\begin{aligned}
\int_{\mathbb{D}}\left|f\left(\varphi_{z}(v)\right)\right|^{p} & \left(1-|v|^{2}\right)^{q+s} d A(v) \\
& =\int_{\mathbb{D}}|f(w)|^{p}\left|\varphi_{z}^{\prime}(w)\right|^{2}\left(1-\left|\varphi_{z}(w)\right|^{2}\right)^{q+s} d A(w) \\
& \geq \frac{1}{\rho(z,|q|+2)} \int_{\mathbb{D}}|f(w)|^{p}\left(1-|w|^{2}\right)^{q}\left(1-\left|\varphi_{z}(w)\right|^{2}\right)^{s} d A(w) .
\end{aligned}
$$

Los siguientes resultados clarificarán la relación entre $L_{0}(p, q, s)$ y $L(p, q, s)$.

Lema 3.1.11 Sean $0<p<\infty,-2<q<\infty$ y $0<s<\infty$. Sean $0<R<1$ y $f \in \mathcal{A}_{q+s}^{p}$. Dado $\varepsilon>0$, existe $0<R^{\prime}<1$ tal que para cada $z \in \mathbb{D}_{R}$

$$
\int_{\mathbb{A}_{R^{\prime}}}|f(w)|^{p} \ln ^{s} \frac{1}{\left|\varphi_{z}(w)\right|} d A_{q}(w)<\varepsilon .
$$

Demostración. Como $f \in \mathcal{A}_{q+s}^{p}$, dado $\varepsilon>0$, existe $0<R^{\prime \prime}<1$ tal que

$$
\int_{\mathbb{A}_{R^{\prime \prime}}}|f(w)|^{p} d A_{q+s}(w)<\varepsilon \frac{(1-R)^{2 s}}{2^{s}} .
$$


Sea $\alpha=.2031 \ldots$ el cero real de $\ln \frac{1}{x}=2(1-x)$. Entonces

$$
\ln \frac{1}{t}<2(1-t), \quad \alpha<t<1,
$$

luego

$$
\ln \frac{1}{r}<1-r^{2}, \quad \alpha<r^{2}<1
$$

Existe $1>R^{\prime}>R^{\prime \prime}>0$, tal que $\frac{2\left(1-|w|^{2}\right)}{(1-R)^{2}}<1-\alpha$ para toda $w \in \mathbb{A}_{R^{\prime}}$. Así $\alpha<\left|\varphi_{z}(w)\right|^{2}<1$, para toda $z \in \mathbb{D}_{R}$ y $w \in \mathbb{A}_{R^{\prime}}$. Entonces

$$
\int_{\mathbb{A}_{R^{\prime}}}|f(w)|^{p} \ln ^{s} \frac{1}{\left|\varphi_{z}(w)\right|} d A_{q}(w) \leq \frac{2^{s}}{(1-R)^{2 s}} \int_{\mathbb{A}_{R^{\prime \prime}}}|f(w)|^{p}\left(1-|w|^{2}\right)^{s} d A_{q}(w)<\varepsilon .
$$

Proposición 3.1.12 Sean $0<p<\infty,-2<q<\infty$ y $0 \leq s<\infty$. Supóngase que $f \in \mathcal{H}$ es tal que $h_{p, q, s}(f)(z)<\infty$ para toda $z \in \mathbb{D}$. Entonces $h_{p, q, s}(f)$ es una función continua en $\mathbb{D}$.

Demostración. Sean $R$ y $R^{\prime}$ como en el Lema 3.1.11. Sea $\left\{b_{n}\right\} \subset \mathbb{D}_{R}$ una sucesión tal que $b_{n} \rightarrow z \in \mathbb{D}_{R}$ cuando $n \rightarrow \infty$. Por (2.3), se observa que

$$
\bigcup_{b \in \mathbb{D}_{R}} \varphi_{b}\left(\mathbb{D}_{R^{\prime}}\right)=\mathbb{D}_{R^{\prime \prime}}, \quad \text { donde } \quad R^{\prime \prime}=\frac{R+R^{\prime}}{1+R R^{\prime}} .
$$

Sea $I_{n}: \mathbb{D}_{R^{\prime \prime}} \rightarrow \mathbb{R}$ definida por

$$
I_{n}(w)=\left|f\left(\varphi_{b_{n}}(w)\right)\right|^{p} \ln ^{s} \frac{1}{|w|}\left|\varphi_{b_{n}}^{\prime}(w)\right|^{2}\left(1-\left|\varphi_{b_{n}}(w)\right|^{2}\right)^{q} \chi_{\varphi_{b_{n}}\left(\mathbb{D}_{R^{\prime}}\right)},
$$

donde $\chi$ denota la función característica. Así $I_{n}(w) \rightarrow I_{z}(w)$ si $n \rightarrow \infty$. Además $\left|I_{n}(w)\right| \leq M \ln ^{s} \frac{1}{|w|}$ para toda $w \in \mathbb{D}_{R^{\prime \prime}}$ y alguna constante $M>0$. Tomando el cambio de variable $w=\varphi_{b_{n}}(v)$ se tiene

$$
\begin{aligned}
\int_{\mathbb{D}_{R^{\prime}}}|f(w)|^{p} & \ln ^{s} \frac{1}{\left|\varphi_{b_{n}}(w)\right|} d A_{q}(w) \\
& =\int_{\varphi_{b_{n}}\left(\mathbb{D}_{R^{\prime}}\right)}\left|f\left(\varphi_{b_{n}}(v)\right)\right|^{p} \ln ^{s} \frac{1}{|v|}\left|\varphi_{b_{n}}^{\prime}(v)\right|^{2}\left(1-\left|\varphi_{b_{n}}(v)\right|^{2}\right)^{q} d A(v) .
\end{aligned}
$$


Entonces, por el teorema de Lebesgue

$$
\lim _{n \rightarrow \infty} \int_{\mathbb{D}_{R^{\prime \prime}}} I_{n}(w) d A(w)=\int_{\mathbb{D}_{R^{\prime \prime}}} I_{z}(w) d A(w) .
$$

Así, por el lema anterior se concluye la prueba.

Proposición 3.1.13 Sean $0<p<\infty,-2<q<\infty$ y $0 \leq s<\infty$. Supóngase que $f \in \mathcal{H}$ es tal que $l_{p, q, s}(f)(z)<\infty$ para toda $z \in \mathbb{D}$. Entonces $l_{p, q, s}(f)$ es una función continua en $\mathbb{D}$.

Demostración. Si $f=0$ en $\mathbb{D}$, es claro que $l_{p, q, s}(f)$ es continua. Por lo tanto supóngase $f \neq 0$, en particular $l_{p, q, s}(f)(0) \neq 0$. Sea $z \in \mathbb{D}$ fijo y sea $\delta>0$ tal que $\overline{\mathbb{D}}_{\delta}(z) \subset \mathbb{D}$. La función $l: \overline{\mathbb{D}} \times \overline{\mathbb{D}}_{\delta}(z) \rightarrow \mathbb{R}$ definida por

$$
(w, \zeta) \rightarrow \frac{\left(1-|\zeta|^{2}\right)^{s}}{|1-\bar{\zeta} w|^{2 s}}
$$

es uniformemente continua en $\overline{\mathbb{D}} \times \overline{\mathbb{D}}_{\delta}(z)$. Así, dado $\epsilon>0$, existe $\rho>0$ tal que si $\left|w^{\prime}-w\right|<\rho \mathrm{y}\left|\zeta^{\prime}-\zeta\right|<\rho$ entonces

$$
\left|l\left(w^{\prime}, \zeta^{\prime}\right)-l(w, \zeta)\right|<\frac{\epsilon}{l_{p, q, s}(f)(0)} .
$$

Luego si $\left|z-z^{\prime}\right|<\rho$ se tiene

$$
\begin{aligned}
& \left|l_{p, q, s}(f)(z)-l_{p, q, s}(f)\left(z^{\prime}\right)\right| \\
& \quad \leq \int_{\mathbb{D}}|f(w)|^{p}\left(1-|w|^{2}\right)^{s}\left|l(w, z)-l\left(w, z^{\prime}\right)\right| d A_{q}(w)<\epsilon .
\end{aligned}
$$

Corolario 3.1.14 Sean $0<p<\infty,-2<q<\infty$ y $0 \leq s<\infty$. Entonces $L_{0}(p, q, s) \subset L(p, q, s) y_{s, 0} \mathcal{A}_{q}^{p} \subset{ }_{s} \mathcal{A}_{q}^{p}$.

Demostración. Es claro que $f=0 \in L_{0}(p, q, s) \cap L(p, q, s)$. Supóngase que $f \neq 0$ y $f \in L_{0}(p, q, s)$. Entonces existe $0<R<1$ tal que $l_{p, q, s}(f)(z)<l_{p, q, s}(f)(0)$ para toda $R<|z|<1$. Por la Proposición 3.1.13, $l_{p, q, s}(f)$ alcanza su máximo finito en $\overline{\mathbb{D}_{R}}$, entonces $f \in L(p, q, s)$. La otra prueba es similar. 
Corolario 3.1.15 Sean $0<p<\infty,-2<q<\infty$ y $0 \leq s<\infty$. Si $f \in L_{0}(p, q, s)$, entonces $l_{p, q, s}(f)$ se puede extender a una función uniformemente continua en $\overline{\mathbb{D}}$. Si $f \in{ }_{s, 0} \mathcal{A}_{q}^{p}$, entonces $h_{p, q, s}(f)$ se puede extender a una función uniformemente continua en $\overline{\mathbb{D}}$.

Considerando las estimaciones en la prueba del Teorema 3.1.6, se obtiene el siguiente resultado.

Corolario 3.1.16 Sean $1 \leq p<\infty,-2<q<\infty$ y $0<s<\infty$ con $-1<q+s$. Entonces ${ }_{s, 0} \mathcal{A}_{q}^{p}$ y $L_{0}(p, q, s)$ son subespacios lineales de Banach de ${ }_{s} \mathcal{A}_{q}^{p}$ y $L(p, q, s)$ respectivamente.

Demostración. Será suficiente con mostrar que ${ }_{s, 0} \mathcal{A}_{q}^{p}$ es un subespacio cerrado de ${ }_{s} \mathcal{A}_{q}^{p}$. Sea $\left\{f_{n}\right\} \subset{ }_{s, 0} \mathcal{A}_{q}^{p}$ una sucesión que converge a $f$. Por el Teorema 3.1.6, $f \in{ }_{s} \mathcal{A}_{q}^{p}$. Por lo tanto, para cualquier $\epsilon>0$, existe $N \in \mathbb{N}$, tal que $\left\|f-f_{n}\right\|_{g}<\frac{\epsilon}{2}$ para toda $n \geq N$. Por hipótesis existe $0<R<1$ tal que si $z \in \mathbb{A}_{R}$ entonces

$$
\int_{\mathbb{D}}\left|f_{N}(w)\right|^{p} g^{s}(w, z) d A_{q}(w)<\frac{\epsilon^{p}}{2^{p}} .
$$

Así

$$
\begin{aligned}
\left(\int_{\mathbb{D}}|f(w)|^{p} g^{s}(w, z) d A_{q}(w)\right)^{\frac{1}{p}} \leq & \left(\int_{\mathbb{D}}\left|f(w)-f_{N}(w)\right|^{p} g^{s}(w, z) d A_{q}(w)\right)^{\frac{1}{p}} \\
& +\left(\int_{\mathbb{D}}\left|f_{N}(w)\right|^{p} g^{s}(w, z) d A_{q}(w)\right)^{\frac{1}{p}} \\
< & \frac{\epsilon}{2}+\frac{\epsilon}{2}=\epsilon .
\end{aligned}
$$

La otra prueba es similar.

Observación: Para $0<p<1$ se obtienen resultados similares con sus respectivas métricas.

3.2. ${ }_{s} \mathcal{A}_{q}^{p}=L(p, q, s)$

En esta sección se prueba la igualdad entre los espacios ${ }_{s} \mathcal{A}_{q}^{p}=L(p, q, s)$. Lo destacado de la prueba de esta igualdad se basa en el lema siguiente, que aparece en $[\mathrm{PeReTo}$. Una primera prueba que se obtuvo de la igualdad de estos espacios e independiente de este lema está en el apéndice. 
Lema 3.2.1 Sean $q(r)$ y $p(r)$ dos funciones integrables no negativas en $[0,1)$. Si existe $\tau^{\prime}$ con $0<\tau^{\prime}<1$ fijo y $C$ una constante positiva tal que $q(r) \leq C p(r)$ para todo $r \in\left[\tau^{\prime}, 1\right)$, entonces para todo $\tau$ con $\tau^{\prime}<\tau \leq 1$ y toda función $h(r)$ no decreciente y no negativa en $[0,1)$, existe una constante $K \geq C$ independiente de $\tau^{\prime}$ y $h$, tal que

$$
\int_{0}^{\tau} h(r) q(r) d r \leq K \int_{0}^{\tau} h(r) p(r) d r
$$

El siguiente Teorema muestra que los espacios métricos o normados ${ }_{s} \mathcal{A}_{q}^{p}$ y $L(p, q, s)$ son equivalentes como conjuntos.

Teorema 3.2.2 Sean $0<p<\infty,-2<q<\infty$ y $0 \leq s<\infty$. Entonces ${ }_{s} \mathcal{A}_{q}^{p}=$ $L(p, q, s)$.

Demostración. El resultado se tendrá si se prueba que existen constantes positivas $K_{1}$ y $K_{2}$ tal que:

$$
\begin{aligned}
\int_{\mathbb{D}}|f(w)|^{p}\left(1-|w|^{2}\right)^{q} & \ln ^{s} \frac{1}{\left|\varphi_{z}(w)\right|} d A(w) \\
& \leq K_{1} \int_{\mathbb{D}}|f(w)|^{p}\left(1-|w|^{2}\right)^{q}\left(1-\left|\varphi_{z}(w)\right|^{2}\right)^{s} d A(w) \\
& \leq K_{2} \int_{\mathbb{D}}|f(w)|^{p}\left(1-|w|^{2}\right)^{q} \ln ^{s} \frac{1}{\left|\varphi_{z}(w)\right|} d A(w) .
\end{aligned}
$$

Con el cambio de variable $w=\varphi_{z}(\lambda)$ basta mostrar

$$
\begin{aligned}
& \int_{\mathbb{D}}\left|f\left(\varphi_{z}(\lambda)\right)\right|^{p}\left(1-\left|\varphi_{z}(\lambda)\right|^{2}\right)^{q} \frac{\left(1-|z|^{2}\right)^{2}}{|1-\bar{z} \lambda|^{4}} \ln ^{s} \frac{1}{|\lambda|} d A(\lambda) \\
\approx & \int_{\mathbb{D}}\left|f\left(\varphi_{z}(\lambda)\right)\right|^{p}\left(1-\left|\varphi_{z}(\lambda)\right|^{2}\right)^{q} \frac{\left(1-|z|^{2}\right)^{2}}{|1-\bar{z} \lambda|^{4}}\left(1-|\lambda|^{2}\right)^{s} d A(\lambda) .
\end{aligned}
$$

Por la Proposición 2.1.1, inciso (3) es equivalente a probar

$$
\begin{aligned}
& \int_{\mathbb{D}}\left|f\left(\varphi_{z}(\lambda)\right)\right|^{p}\left(1-|\lambda|^{2}\right)^{q} \frac{\left(1-|z|^{2}\right)^{q+2}}{|1-\bar{z} \lambda|^{4+2 q}} \ln ^{s} \frac{1}{|\lambda|} d A(\lambda) \\
\approx & \int_{\mathbb{D}}\left|f\left(\varphi_{z}(\lambda)\right)\right|^{p}\left(1-|\lambda|^{2}\right)^{q+s} \frac{\left(1-|z|^{2}\right)^{q+2}}{|1-\bar{z} \lambda|^{4+2 q}} d A(\lambda) .
\end{aligned}
$$

La función $H: \mathbb{D} \rightarrow \mathbb{R}$ dada por

$$
H(\lambda)=\left|f\left(\varphi_{z}(\lambda)\right)\right|^{p} \frac{\left(1-|z|^{2}\right)^{q+2}}{|1-\bar{z} \lambda|^{4+2 q}}
$$


3.2. ${ }_{s} \mathcal{A}_{q}^{p}=L(p, q, s)$

es subarmónica. Usando esta notación y coordenadas polares en (3.10), se tiene que probar

$$
\int_{0}^{1}\left(1-r^{2}\right)^{q} r \ln ^{s} \frac{1}{r} \int_{0}^{2 \pi} H\left(r e^{i \theta}\right) d \theta d r \approx \int_{0}^{1}\left(1-r^{2}\right)^{q+s} r \int_{0}^{2 \pi} H\left(r e^{i \theta}\right) d \theta d r .
$$

Puesto que $H\left(r e^{i \theta}\right)$ es una función subarmónica no negativa, la función

$$
h(r)=\int_{0}^{2 \pi} H\left(r e^{i \theta}\right) d \theta
$$

es una función no decreciente y no negativa, y las funciones $q(r)=\left(1-r^{2}\right)^{q} r \ln ^{s} \frac{1}{r}$ y $\left(1-r^{2}\right)^{q+s} r$ son funciones continuas en $[0,1)$ (se define $q(0)=\lim _{r \rightarrow 0^{+}} q(r)=0$ ). Sea $\tau^{\prime}=0.450754 \ldots$ el cero de la ecuación $1-x^{2}=-\ln x$. Si $r \in\left[\tau^{\prime}, 1\right)$ entonces $p(r) \leq q(r)$, y como $1-x^{2} \leq-2 \ln x$ para toda $x \in[0,1)$ entonces $p(r) \leq 2 q(r)$ cuando $r \in\left[\tau^{\prime}, 1\right)$. Luego se cumplen las hipótesis del Lema 3.2.1 y por ello existen las constantes $K_{1}$ y $K_{2}$ que satisfacen (3.9).

Proposición 3.2.3 Sean $0<p<\infty$ y $0 \leq s<\infty$. Entonces, para $-1<q<\infty$,

$$
H^{p} \subset \bigcap_{-1<q, 0 \leq s} s, 0 \mathcal{A}_{q}^{p} .
$$

Demostración. Sean $f \in H^{p}$ y $\epsilon>0$. Se define

$$
M=\sup _{0 \leq r<1} \int_{0}^{2 \pi}\left|f\left(r e^{i \theta}\right)\right|^{p} d \theta<\infty .
$$

Entonces existe $0<r<1$ tal que

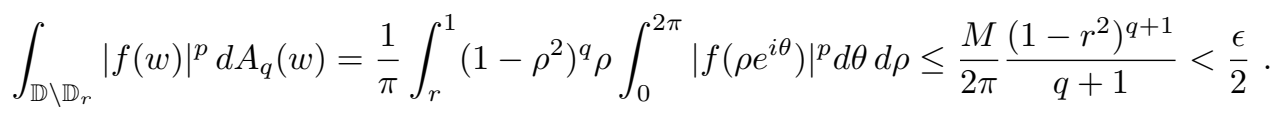

Como $\left(1-\left|\varphi_{z}(w)\right|^{2}\right)^{s}<1$, se descompone

$$
l_{p, q, s}(f)(z) \leq \operatorname{máx}_{w \in \mathbb{D}_{r}}|f(w)|^{p} \int_{\mathbb{D}_{r}}\left(1-\left|\varphi_{z}(w)\right|^{2}\right)^{s} d A_{q}(z)+\int_{\mathbb{D}_{\mathbb{D}} \mathbb{D}_{r}}|f(z)|^{p} d A_{q}(z),
$$

entonces por el Lema 2.1.5 se concluye la prueba tomando el límite cuando $|z| \rightarrow 1^{-}$. 


\subsection{Inclusiones Estrictas}

En esta sección se probarán las inclusiones estrictas entre los espacios de Bergman pesados para $0<s \leq 1$. Para esto se necesitan los siguientes dos lemas.

El primero es debido a A. Zygmund, (ver $[\mathrm{Zy}]$ ).

Lema 3.3.1 Sea $0<p<\infty$. Si $\left\{n_{k}\right\}$ es una sucesión creciente de enteros positivos que satisface $\frac{n_{k+1}}{n_{k}} \geq \lambda>1$, para todo $k \in \mathbb{N}$, entonces existe una constante $A>0$, que depende sólo de p y $\lambda$, tal que

$$
\frac{1}{A}\left(\sum_{k=1}^{\infty}\left|a_{k}\right|^{2}\right)^{\frac{1}{2}} \leq\left(\frac{1}{2 \pi} \int_{0}^{2 \pi}\left|\sum_{k=1}^{\infty} a_{k} e^{i n_{k} \theta}\right|^{p} d \theta\right)^{\frac{1}{p}} \leq A\left(\sum_{k=1}^{\infty}\left|a_{k}\right|^{2}\right)^{\frac{1}{2}},
$$

para cualesquiera números $a_{k}, k \in \mathbb{N}$.

Para $n \in \mathbb{N}$, se define

$$
I_{n}=\left\{k \in \mathbb{N}: 2^{n} \leq k<2^{n+1}\right\} .
$$

El siguiente lema fue probado por Mateljevic y Pavlovic en [MaPa].

Lema 3.3.2 Sean $\alpha>0$ y $p>0$. Sea $f(x)=\sum_{n=1}^{\infty} a_{n} x^{n}$, con $0 \leq x<1$ y $a_{n} \geq 0$ para cada $n \in \mathbb{N}$. Entonces existe una constante $K>0$ que depende sólo de $p$ y $\alpha$ tal que

$$
\frac{1}{K} \sum_{n=0}^{\infty} \frac{t_{n}^{p}}{2^{n \alpha}} \leq \int_{0}^{1}(1-x)^{\alpha-1} f(x)^{p} d x \leq K \sum_{n=0}^{\infty} \frac{t_{n}^{p}}{2^{n \alpha}}
$$

donde $t_{n}=\sum_{k \in I n} a_{k}$.

Teorema 3.3.3 Sean $0<p<\infty,-2<q<\infty$ y $0<s<1$ con $-1<q+s$. Si $f \in \mathcal{H}$ tiene una expansión en serie de potencias dada por

$$
f(w)=\sum_{n=0}^{\infty} a_{n} w^{n}
$$

y además

$$
\int_{0}^{1}\left(\sum_{n=1}^{\infty}\left|a_{n}\right| r^{n}\right)^{p}\left(1-r^{2}\right)^{q+s} d r<\infty
$$

entonces $f \in{ }_{s, 0} \mathcal{A}_{q}^{p}$. 
Demostración. Sea $0<R<1$. Se considera la siguiente estimación

$$
\begin{aligned}
l_{p, q, s}(z) \leq & \sup _{w \in \mathbb{D}_{R}}|f(w)|^{p} \int_{\mathbb{D}_{R}}\left(1-\left|\varphi_{z}(w)\right|^{2}\right)^{s} d A_{q}(w) \\
& +\frac{1}{\pi} \int_{R}^{1}\left(\sum_{n=1}^{\infty}\left|a_{n}\right| r^{n}\right)^{p}\left(1-r^{2}\right)^{q+s} r\left(1-|z|^{2}\right)^{s} \int_{0}^{2 \pi} \frac{d \theta}{\left|1-\bar{z} r e^{i \theta}\right|^{2 s}} d r
\end{aligned}
$$

Se concluye la prueba aplicando los Lemas 2.1.5 y 1.4.2 y tomando el límite cuando $|z| \rightarrow 1^{-}$.

Corolario 3.3.4 Sean $0<p<\infty,-2<q<\infty$ y $0<s \leq 1$, con $-1<q+s$. Supóngase que $f \in \mathcal{H}$ tiene una expansión en serie de potencias dada por

$$
f(w)=\sum_{n=0}^{\infty} a_{n} w^{n} .
$$

Si

$$
\sum_{n=0}^{\infty} \frac{1}{2^{n(q+s+1)}}\left(\sum_{k \in I_{n}}\left|a_{k}\right|\right)^{p}<\infty
$$

entonces $f \in{ }_{s, 0} \mathcal{A}_{q}^{p}$.

Demostración. Es una consecuencia inmediata del Teorema 3.3.3, la condición (3.11) y el Lema 3.3.2.

Una sucesión $\left\{a_{n}\right\}$ tiene saltos de Hadamard de longitud $\lambda>1$, si existe una sucesión creciente $\left\{n_{k}\right\} \subset \mathbb{N}$ tal que

$$
a_{n}= \begin{cases}0 & \text { si } n \neq n_{k} \\ a_{n_{k}} & \text { si } n=n_{k},\end{cases}
$$

con

$$
\frac{n_{k+1}}{n_{k}} \geq \lambda>1, \quad \text { para } k \in \mathbb{N} .
$$

Dada $f \in \mathcal{H}$ con expansión en serie de potencias

$$
f(w)=\sum_{n=0}^{\infty} a_{n} w^{n}
$$

se dice que $f$ es Lacunaria si la sucesión de coeficientes $\left\{a_{n}\right\}$ tiene saltos de Hadamard de longitud $\lambda$, para algún $\lambda>1$. Se escribe simplemente

$$
f(w)=\sum_{k=0}^{\infty} a_{k} w^{n_{k}} .
$$


Se observa que el número de coeficientes de Taylor $a_{j}$ es a lo más $\left[\log _{\lambda} 2\right]+1$ cuando $n_{j} \in I_{k}$. El siguiente teorema caracteriza las series Lacunarias con saltos de Hadamard en los espacios ${ }_{s, 0} \mathcal{A}_{q}^{p} \mathrm{y}_{s} \mathcal{A}_{q}^{p}$.

Teorema 3.3.5 Sean $0<p<\infty,-2<q<\infty$ y $0<s \leq 1$ con $-1<q+s$. Supóngase que $f \in \mathcal{H}$ es Lacunaria con serie de potencias dada por

$$
f(z)=\sum_{k=0}^{\infty} a_{k} z^{n_{k}} .
$$

Entonces las siguientes afirmaciones son equivalentes:

i) $f \in{ }_{s, 0} \mathcal{A}_{q}^{p}$;

ii) $f \in{ }_{s} \mathcal{A}_{q}^{p}$;

iii) $f \in \mathcal{A}_{q+s}^{p}$;

iv) la serie

$$
\sum_{k=0}^{\infty} \frac{1}{2^{k(1+q+s)}}\left(\sum_{n_{j} \in I_{k}}\left|a_{n_{j}}\right|^{2}\right)^{\frac{p}{2}}
$$

es convergente.

Demostración. Sea $\lambda>1$ la longitud del salto de Hadamard de los coeficientes de la serie. Por el Corolario 3.1.14, se sigue que i) implica ii). Ahora ${ }_{s} \mathcal{A}_{q}^{p} \subset \mathcal{A}_{q+s}^{p}$, así ii) implica iii). Se ve ahora que iii) implica iv). Por los Lemas 3.3.1 y 3.3.2, existen $A>0$ y $K>0$, tales que

$$
\begin{aligned}
\int_{\mathbb{D}}|f(w)|^{p} d A_{q+s}(w) & =\int_{\mathbb{D}}\left|\sum_{k=0}^{\infty} a_{k} z^{n_{k}}\right|^{p} d A_{q+s}(w) \\
& =\frac{1}{\pi} \int_{0}^{1} \int_{0}^{2 \pi}\left|\sum_{k=0}^{\infty} a_{k} r^{n_{k}} e^{i n_{k} \theta}\right|^{p}\left(1-r^{2}\right)^{q+s} r d \theta d r \\
& \geq \frac{2}{A^{p}} \int_{0}^{1}\left(\sum_{k=0}^{\infty}\left|a_{k}\right|^{2} r^{2 n_{k}}\right)^{\frac{p}{2}} r\left(1-r^{2}\right)^{q+s} d r \\
& \geq \frac{1}{K A^{p}} \sum_{k=0}^{\infty} \frac{1}{2^{k(q+s+1)}}\left(\sum_{n_{j} \in I_{k}}\left|a_{j}\right|^{2}\right)^{\frac{p}{2}}
\end{aligned}
$$

y la serie (3.12) es convergente.

Se prueba que iv) implica i). Como

$$
\left(\sum_{n_{j} \in I_{n}}\left|a_{j}\right|\right)^{p} \leq 2^{\frac{p}{2}}\left(\left[\log _{\lambda} 2\right]+1\right)^{\frac{p}{2}}\left(\sum_{n_{j} \in I_{k}}\left|a_{j}\right|^{2}\right)^{\frac{p}{2}}
$$

por el Corolario 3.3.4 se tiene que $f \in \in_{s, 0} \mathcal{A}_{q}^{p}$. 
Corolario 3.3.6 Sean $0<p<\infty,-2<q<\infty$ y $0<s<1$ con $-1<q+s$. Entonces las inclusiones

$$
{ }_{s} \mathcal{A}_{q}^{p} \subset \bigcap_{s<t \leq 1}{ }_{t} \mathcal{A}_{q}^{p} \quad \text { y } \quad{ }_{s, 0} \mathcal{A}_{q}^{p} \subset \bigcap_{s<t \leq 1}{ }_{t, 0} \mathcal{A}_{q}^{p}
$$

son estrictas.

Demostración. Se define $f: \mathbb{D} \rightarrow \mathbb{C}$ por

$$
f(w)=\sum_{n=0}^{\infty} 2^{\frac{n(q+s+1)}{p}} w^{2^{n}} .
$$

Entonces, $f \in \mathcal{H}$ y para $s<t \leq 1$,

$$
\sum_{n=0}^{\infty} \frac{1}{2^{n(1+q+t)}}\left(\left|a_{n}\right|^{2}\right)^{\frac{p}{2}}=\sum_{n=0}^{\infty} \frac{2^{n(q+s+1)}}{2^{n(1+q+t)}}=\sum_{n=0}^{\infty} \frac{1}{2^{(t-s) n}}<\infty
$$

y

$$
\sum_{n=0}^{\infty} \frac{1}{2^{n(1+q+s)}}\left(\left|a_{n}\right|^{2}\right)^{\frac{p}{2}}=\sum_{n=0}^{\infty} \frac{2^{n(q+s+1)}}{2^{n(1+q+s)}}=\sum_{n=0}^{\infty} 1=\infty .
$$

Por lo tanto por el Teorema 3.3.5

$$
f \in \bigcap_{s<t \leq 1}{ }_{t, 0} \mathcal{A}_{q}^{p} \quad \text { pero } \quad f \notin{ }_{s, 0} \mathcal{A}_{q}^{p} .
$$

Se recuerda que si $0<t<s<1$ entonces ${ }_{t} \mathcal{A}_{q}^{p}$ es un subconjunto propio de ${ }_{s} \mathcal{A}_{q}^{p}$. El siguiente resultado se usará para mostrar que ${ }_{t} \mathcal{A}_{q}^{p}$ no es un subespacio cerrado de ${ }_{s} \mathcal{A}_{q}^{p}$.

Lema 3.3.7 Sean $0<s<1$ y $f(w)=\sum_{k=0}^{\infty} a_{k} w^{k}$. Entonces

$$
\int_{\mathbb{D}}\left(\sum_{k=0}^{\infty}\left|a_{k}\right||w|^{k}\right)^{p}\left(1-\left|\varphi_{z}(w)\right|^{2}\right)^{s} d A_{q}(w) \leq C(p, q, s) \sum_{k=0}^{\infty} \frac{t_{n}^{p}}{2^{n(q+s+1)}},
$$

donde $t_{n}=\sum_{k \in I_{n}}\left|a_{k}\right|$. 
Demostración. Se observa que para $0<s<1$ se tiene

$$
\begin{aligned}
I(z) & =\int_{\mathbb{D}}\left(\sum_{k=0}^{\infty}\left|a_{k}\right||w|^{k}\right)^{p}\left(1-\left|\varphi_{z}(w)\right|^{2}\right)^{s}\left(1-|w|^{2}\right)^{q} d A(w) \\
& =\frac{1}{\pi} \int_{0}^{1} \int_{0}^{2 \pi}\left(\sum_{k=0}^{\infty}\left|a_{k}\right| r^{k}\right)^{p}\left(1-r^{2}\right)^{q} \cdot r \cdot \frac{\left(1-|z|^{2}\right)^{s}\left(1-r^{2}\right)^{s}}{\left|1-z r e^{-i \theta}\right|^{2 s}} d \theta d r \\
& \leq \frac{2^{s}}{\pi} \int_{0}^{1}\left(\sum_{k=0}^{\infty}\left|a_{k}\right| r^{k}\right)^{p}\left(1-r^{2}\right)^{q+s} \cdot r \cdot \int_{0}^{2 \pi} \frac{1}{\left|1-(z r) e^{-i \theta \mid}\right|^{s}} d \theta d r \\
& \leq C_{1}(s) \int_{0}^{1}\left(\sum_{k=0}^{\infty}\left|a_{k}\right| r^{k}\right)^{p}\left(1-r^{2}\right)^{q+s} d r
\end{aligned}
$$

donde se obtiene la última desigualdad por el Teorema 1.4.2. Por el Lema 3.3.2 existe una constante $C_{2}(p, q, s)$ tal que

$$
I(z) \leq C_{1}(s) \cdot C_{2}(p, q, s) \sum_{n=0}^{\infty} \frac{t_{n}^{p}}{2^{n(q+s+1)}} .
$$

Teorema 3.3.8 Sean $0<p<\infty,-1<q<\infty$ y $0<t<s<1$. Entonces el subespacio ${ }_{t} \mathcal{A}_{q}^{p}$ no es un subespacio cerrado de ${ }_{s} \mathcal{A}_{q}^{p}$.

Demostración. Se prueba para $1 \leq p<\infty$, el otro caso es análogo. Ya se probó la inclusión ${ }_{t} \mathcal{A}_{q}^{p} \subset{ }_{s} \mathcal{A}_{q}^{p}$. Considerando la siguiente serie Lacunaria y su sucesión de sumas parciales

$$
f(z)=\sum_{n=0}^{\infty} 2^{\frac{n(q+t+1)}{p}} z^{2^{n}} \quad \text { y } \quad f_{n}(z)=\sum_{n=0}^{k} 2^{\frac{n(q+t+1)}{p}} z^{2^{n}},
$$

entonces $\left\{f_{n}\right\} \subset{ }_{t} \mathcal{A}_{q}^{p} \cap_{s} \mathcal{A}_{q}^{p}$ y converge a la función $f$ en la norma $\|\cdot\|_{\varphi, p, q, s}$. En efecto, por Lema 3.3.7, para $0<s<1$ existe una constante $C(p, q, s)$ tal que

$$
\begin{aligned}
I(z) & =\int_{\mathbb{D}}\left|f(w)-f_{n}(w)\right|^{p}\left(1-\left|\varphi_{z}(w)\right|^{2}\right)^{s} d A_{q}(w) \\
& \leq \int_{\mathbb{D}}\left(\sum_{m=n}^{\infty} 2^{\frac{n(q+t+1)}{p}}|w|^{2^{n}}\right)^{p}\left(1-\left|\varphi_{z}(w)\right|^{2}\right)^{s} d A_{q}(w) \\
& \leq C(p, q, s) \sum_{m=n}^{\infty} \frac{2^{m(q+t+1)}}{2^{m(q+s+1)}} \\
& =C(p, q, s) \sum_{m=n}^{\infty} \frac{1}{2^{m(s-t)}} .
\end{aligned}
$$


Como $t<s$, entonces $\sum_{m=n}^{\infty} \frac{1}{2^{m(s-t)}}$ es una serie convergente y así $f_{n}$ converge a $f$ en la mencionada norma. En particular $\left\{f_{n}\right\}$ es una sucesión de Cauchy con respecto a la norma $\|\cdot\|_{\varphi, p, q, s}$. Por el Teorema 3.3.5 $f \notin_{t} \mathcal{A}_{q}^{p}$ pues

$$
\sum_{k=0}^{\infty} \frac{2^{n(q+t+1)}}{2^{n(q+t+1)}}=\infty .
$$

Para $S \subset \mathcal{H}$, se define su conjunto de primitivas $S$, como

$$
\mathcal{P}(S)=\left\{h \in \mathcal{H}: h^{\prime} \in S\right\} .
$$

Se observa que si $f \in{ }_{s} \mathcal{A}_{q}^{p}$ para $0<p<\infty,-2<q<\infty, 0 \leq s<\infty$, y $h$ es una primitiva de $f$, entonces $h \in F(p, q, s)$. Por definición $h \in F(p, q, s)$ si:

$$
\sup _{z \in \mathbb{D}} \int_{\mathbb{D}}\left|h^{\prime}(z)\right|^{p} g^{s}(z, a) d A_{q}(z)<\infty,
$$

es decir, $\mathcal{P}\left({ }_{s} \mathcal{A}_{q}^{p}\right)=F(p, q, s)$. Entonces por el Teorema 4.2.1 de J. Rättyä se tiene una caracterización del espacio ${ }_{s} \mathcal{A}_{q}^{p}$ en términos de las derivadas superiores con $1<p<\infty$. Teorema 3.3.9 $[R a]($ Theorem 4.2.1) Sean $f \in \mathcal{H}$ y $h$ una primitiva de $f$. Sean $1<p<\infty,-2<q<\infty, 0 \leq s<\infty$ y $n \in \mathbb{N}$ con $-1<q+s$. Entonces las siguientes condiciones son equivalentes:

a) $h \in F(p, q, s)$;

b) $f \in{ }_{s} \mathcal{A}_{q}^{p}$;

c) $\sup _{a \in \mathbb{D}} \int_{\mathbb{D}}\left|f^{(n-1)}(z)\right|^{p}\left(1-|z|^{2}\right)^{n p-p}\left(1-\left|\varphi_{a}(z)\right|^{2}\right)^{s} d A_{q}(z)<\infty$;

d) $\sup _{a \in \mathbb{D}} \int_{\mathbb{D}}\left|f^{(n-1)}\left(\varphi_{a}(z)\right)\right|^{p}\left|\varphi_{a}^{\prime}(z)\right|^{n p-p+q+2}\left(1-|z|^{2}\right)^{n p-p+s} d A_{q}(z)<\infty$;

e) $\sup _{a \in \mathbb{D}} \int_{\mathbb{D}}\left|f^{(n-1)}(z)\right|^{p}\left(1-|z|^{2}\right)^{n p-p} g^{s}(z, a) d A_{q}(z)<\infty$.

Por el Teorema 4.2.2 de J. Rättyä y lo realizado hasta ahora se tienen las siguientes relaciones entre los espacios ${ }_{s} \mathcal{A}_{q}^{p}$ y $F(p, q, s)$.

Teorema 3.3.10 [Ra](Theorem 4.2.2) Sea $h \in \mathcal{H}$. Sean $1<p<\infty,-2<q<\infty y$ $0 \leq s<\infty$ con $-1<q+s-p$. Entonces las siguientes condiciones son equivalemtes:

a) $h \in F(p, q, s)$;

b) $\sup _{a \in \mathbb{D}} \int_{\mathbb{D}}|h(z)|^{p}\left(1-|z|^{2}\right)^{-p+q}\left(1-\left|\varphi_{a}(z)\right|^{2}\right)^{s} d A_{q}(z)<\infty$;

c) $\sup _{a \in \mathbb{D}} \int_{\mathbb{D}}|h(z)|^{p}\left(1-|z|^{2}\right)^{-p+q} g^{s}(z, a) d A_{q}(z)<\infty$. 
Corolario 3.3.11 Para $h \in \mathcal{H}$, sean $1<p<\infty,-2<q<\infty$ y $0 \leq s<\infty$ con $-1<q+s-p y-2<q-p$. Entonces $F(p, q, s)={ }_{s} \mathcal{A}_{q-p}^{p}$. En particular,

$$
\mathcal{P}(F(p, q, s))=\mathcal{P}\left({ }_{s} \mathcal{A}_{q-p}^{p}\right)=F(p, q-p, s) .
$$




\section{Capítulo 4}

\section{El operador de Bergman $P_{\beta}$ en los espacios $L(p, q, s)={ }_{s} \mathcal{A}_{q}^{p}$}

Es bien conocido que si $-1<q, \beta<\infty$ y $1 \leq p<\infty$ entonces la proyección de Bergman $P_{\beta}$ es un operador acotado de $L^{p}\left(\mathbb{D}, d A_{q}\right)$ sobre el espacio de Bergman $\mathcal{A}_{q}^{p}$ si y sólo si $q+1<(\beta+1) p$, ver Teorema 1.5.5. En este capítulo se estudia el operador de Bergman $P_{\beta}$ del espacio $L^{p}\left(\mathbb{D}, d A_{q}\right)$ en los espacios de Bergman pesados ${ }_{s} \mathcal{A}_{q}^{p}=L(p, q, s)$ y además se prueba que es un operador acotado para ciertos valores de $\beta, p, q$ y $s$, que en particular satisfacen $q+1 \geq(\beta+1) p$, así el Teorema 1.5.5 se puede extender en el sentido de la suficiencia, gracias a la adición del peso $1-\left|\varphi_{z}(w)\right|^{2}$.

\subsection{Una Estimación Integral}

Para estudiar el operador de Bergman $P_{\beta}$ sobre los espacios ${ }_{s} \mathcal{A}_{q}^{p}$, es necesaria una estimación integral que es citada sin prueba en [OrFa]. Se prueban varios resultados para dar una demostración siguiendo las ideas en [OrFa1]. Como se verá, la prueba dista de ser directa.

Para $z, \zeta \in \mathbb{C}$ sea

$$
d(\zeta, z)=|\bar{z}(z-\zeta)|+|\bar{\zeta}(\zeta-z)|
$$

una pseudo-distancia no isotrópica; restringida a esferas con centro en el origen si es una distancia. La siguiente proposición muestra por qué no es una distancia.

Proposición 4.1.1 Existe una constante $C>0$ tal que

$$
d(\zeta, z) \leq C(d(\zeta, w)+d(w, z))
$$


para todo $\zeta, z, w \in \overline{\mathbb{D}}$, esto es $d(\zeta, z) \preceq d(\zeta, w)+d(w, z)$.

Demostración. Se procede por reducción al absurdo. Supóngase que para cada $n \in \mathbb{N}$ existen $z_{n}, w_{n}, \zeta_{n} \in \overline{\mathbb{D}}$ tales que

$$
d\left(z_{n}, \zeta_{n}\right)>n\left(d\left(z_{n}, w_{n}\right)+d\left(w_{n}, \zeta_{n}\right)\right) .
$$

Se puede asumir por Bolzano-Weierstrass que $z_{n} \rightarrow z, w_{n} \rightarrow w$ y $\zeta_{n} \rightarrow \zeta$, con $z, w \mathrm{y}$ $\zeta \in \overline{\mathbb{D}}$. Como

$$
d\left(z_{n}, \zeta_{n}\right)>n \operatorname{máx}\left\{d\left(z_{n}, w_{n}\right), d\left(w_{n}, \zeta_{n}\right)\right\}
$$

entonces $z=\zeta=w$. Ahora, sin pérdida de generalidad, supóngase que $|z|=R$. Entonces existe $N>3$ tal que para $n \geq N$

$$
\begin{aligned}
3 R\left|\zeta_{n}-z_{n}\right| \geq d\left(z_{n}, \zeta_{n}\right) & \geq n\left(\left(\left|z_{n}\right|+\left|w_{n}\right|\right)\left|z_{n}-w_{n}\right|+\left(\left|w_{n}\right|+\left|\zeta_{n}\right|\right)\left|w_{n}-\zeta_{n}\right|\right) \\
& \geq n R\left(\left|z_{n}-w_{n}\right|+\left|w_{n}-\zeta_{n}\right|\right) .
\end{aligned}
$$

Así

$$
3\left(\left|z_{n}-w_{n}\right|+\left|w_{n}-\zeta_{n}\right|\right) \geq 3\left|\zeta_{n}-z_{n}\right| \geq n R\left(\left|z_{n}-w_{n}\right|+\left|w_{n}-\zeta_{n}\right|\right)
$$

y se obtiene una contradicción.

Lema 4.1.2 Sean $\zeta, z \in \mathbb{D}$ y $C>0$ como en la Proposición 4.1.1. Si

$$
\Omega=\left\{\eta \in \mathbb{D}: d(\eta, z) \leq \frac{d(\zeta, z)}{2 C}\right\}
$$

entonces

$$
|1-\bar{\eta} z| \preceq|1-\bar{\zeta} z| \preceq|1-\bar{\eta} \zeta|, \quad \text { para } \eta \in \Omega
$$

Demostración. Primero se observa que para $\zeta, z \in \mathbb{D}$

$$
|1-\bar{\zeta} z| \approx 1-|\zeta|^{2}+d(\zeta, z) \approx 1-|z|^{2}+d(\zeta, z) .
$$

En efecto, se tiene que

$$
\begin{aligned}
|1-\bar{\zeta} z| & =|1-\bar{\zeta} \zeta+\bar{\zeta} \zeta-\bar{\zeta} z| \leq|1-\bar{\zeta} \zeta|+|\bar{\zeta}(z-\zeta)| \\
& =\left.|1-| \zeta\right|^{2}|+| \zeta|| \zeta-\left.z|\leq 1-| \zeta\right|^{2}+(|\zeta|+|z|)|\zeta-z| \\
& =1-|\zeta|^{2}+d(\zeta, z) .
\end{aligned}
$$

Por otra parte, $1-|z|^{2} \leq 2(1-|z|) \leq 2|1-\bar{\zeta} z|$. Además

$$
\begin{aligned}
|z-\zeta| & =|z-z \zeta \bar{z}+z \zeta \bar{z}-\zeta|=\left|z(1-\bar{z} \zeta)+\zeta\left(|z|^{2}-1\right)\right| \\
& \leq|z||1-\bar{z} \zeta|+|\zeta|\left(1-|z|^{2}\right) \\
& \leq 3|1-\bar{z} \zeta|
\end{aligned}
$$


y así se ha probado (4.3).

Ahora, se mostrará que

$$
|1-\bar{\eta} z| \preceq|1-\bar{\zeta} z| \preceq|1-\bar{\eta} \zeta|, \quad \text { para cada } \eta \in \Omega .
$$

Como $\eta \in \Omega$, por (4.3) se tiene

$$
|1-\bar{\eta} z| \approx 1-|z|^{2}+d(\eta, z) \preceq 1-|z|^{2}+d(z, \zeta) \approx|1-\bar{\zeta} z| .
$$

En el otro lado se observa que

$$
d(z, \zeta) \leq C(d(z, \eta)+d(\eta, \zeta)) \leq C\left(\frac{d(z, \zeta)}{2 C}+d(\eta, \zeta)\right)
$$

y de aquí

$$
d(z, \zeta) \leq 2 C d(\eta, \zeta)
$$

Así

$$
|1-\bar{\zeta} z| \approx 1-|\zeta|^{2}+d(z, \zeta) \preceq 1-|\zeta|^{2}+d(\eta, \zeta) \approx|1-\bar{\eta} \zeta| .
$$

y se tiene la prueba.

Para el caso de ciertos exponentes el siguiente resultado permite una estimación similar al Teorema 1.4.2.

Lema 4.1.3 Supóngase que $-1<t_{2}<\infty, 0 \leq t_{1}<2+t_{2}<\infty y-1 \leq t_{0}<t_{2}<$ $t_{0}+t_{1}<\infty$. Entonces

$$
\int_{\mathbb{D}} \frac{\left(1-|\eta|^{2}\right)^{t_{2}}}{|1-\bar{\eta} z|^{2+t_{0}}|1-\bar{\eta} \zeta|^{t_{1}}} d A(\eta) \preceq \frac{1}{|1-\bar{\zeta} z|^{t_{0}+t_{1}-t_{2}}} .
$$

Demostración. Aplicando el Lema 4.1.2, se tiene

$$
|1-\bar{\zeta} z|+1-|\eta|^{2} \preceq|1-\bar{\eta} \zeta|,
$$

ya que $|1-\bar{\zeta} z| \preceq|1-\bar{\eta} \zeta|$ para todo $\eta \in \Omega$ y $1-|\eta|^{2} \leq 2|1-\bar{\eta} \zeta|$. Ahora $|1-\bar{\zeta} z| \preceq|1-\bar{\eta} z|$ para todo $\eta \in \mathbb{D} \backslash \Omega$ y $1-|\eta|^{2} \leq 2|1-\bar{\eta} \zeta|$, entonces

$$
(|1-\bar{\zeta} z|+|1-\bar{\eta} z|)^{2+t_{0}}\left(1-|\eta|^{2}\right)^{t_{1}} \preceq|1-\bar{\eta} z|^{2+t_{0}}|1-\bar{\eta} \zeta|^{t_{1}}
$$

Se usa la partición $\mathbb{D}=\Omega \cup(\mathbb{D} \backslash \Omega)$ para estimar la integral $I(z, \zeta)$, así

$$
\begin{aligned}
I(z, \zeta):= & \int_{\mathbb{D}} \frac{\left(1-|\eta|^{2}\right)^{t_{2}}}{|1-\bar{\eta} z|^{2+t_{0}}|1-\bar{\eta} \zeta|^{t_{1}}} d A(\eta) \\
\preceq & \int_{\Omega} \frac{\left(1-|\eta|^{2}\right)^{t_{2}}}{|1-\bar{\eta} z|^{2+t_{0}}\left(|1-\bar{\zeta} z|+1-|\eta|^{2}\right)^{t_{1}}} d A(\eta) \\
& \quad+\int_{\mathbb{D} \backslash \Omega} \frac{\left(1-|\eta|^{2}\right)^{t_{2}-t_{1}}}{(|1-\bar{\zeta} z|+|1-\bar{\eta} z|)^{2+t_{0}}} d A(\eta) .
\end{aligned}
$$


Por un cambio de variable en coordenadas polares se obtiene

$$
\begin{aligned}
I(z, \zeta) \preceq & \int_{0}^{1} \int_{0}^{2 \pi} \frac{\left(1-r^{2}\right)^{t_{2}} r}{\left|1-r z e^{-i \theta}\right|^{2+t_{0}}\left(|1-\bar{\zeta} z|+1-r^{2}\right)^{t_{1}}} d \theta d r \\
& +\int_{0}^{1} \int_{0}^{2 \pi} \frac{\left(1-r^{2}\right)^{t_{2}-t_{1}} r}{\left(|1-\bar{\zeta} z|+\left|1-r z e^{-i \theta}\right|\right)^{2+t_{0}}} d \theta d r .
\end{aligned}
$$

Por el Teorema 1.4.2

$$
\int_{0}^{2 \pi} \frac{d \theta}{\left|1-r z e^{-i \theta}\right|^{2+t_{0}}} \approx \frac{1}{\left(1-r^{2}|z|^{2}\right)^{1+t_{0}}}
$$

y de la estimación

$$
(1+|1-\bar{\zeta} z|)\left|1-\frac{r z}{1+|1-\bar{\zeta} z|} e^{-i \theta}\right|=|1+| 1-\bar{\zeta} z\left|-r z e^{-i \theta}\right| \leq|1-\bar{\zeta} z|+\left|1-r z e^{i \theta}\right|
$$

se tiene

$$
\begin{aligned}
\int_{0}^{2 \pi} \frac{d \theta}{\left(|1-\bar{\zeta} z|+\left|1-r z e^{-i \theta}\right|\right)^{2+t_{0}}} & \leq \frac{1}{(1+|1-\bar{\zeta} z|)^{2+t_{0}}} \int_{0}^{2 \pi} \frac{d \theta}{\left|1-\frac{r z}{1+|1-\bar{\zeta} z|} e^{-i \theta}\right|^{2+t_{0}}} \\
& \preceq \frac{1}{(1+|1-\bar{\zeta} z|)^{2+t_{0}}} \frac{1}{\left(1-\frac{r^{2}|z|^{2}}{(1+|1-\bar{\zeta} z|)^{2}}\right)^{1+t_{0}}} \\
& \preceq \frac{1}{\left((1+|1-\bar{\zeta} z|)^{2}-r^{2}|z|^{2}\right)^{1+t_{0}}} \\
& \leq \frac{1}{\left(1+|1-\bar{\zeta} z|-r^{2}|z|^{2}\right)^{1+t_{0}}}
\end{aligned}
$$

Por lo tanto

$$
\begin{aligned}
& I(z, \zeta) \preceq \int_{0}^{1} \frac{\left(1-r^{2}\right)^{t_{2}} r}{\left(1-|z|^{2} r^{2}\right)^{1+t_{0}}\left(|1-\bar{\zeta} z|+1-r^{2}\right)^{t_{1}}} d r \\
& +\int_{0}^{1} \frac{\left(1-r^{2}\right)^{t_{2}-t_{1}} r}{\left(|1-\bar{\zeta} z|+1-|z|^{2} r^{2}\right)^{1+t_{0}}} d r .
\end{aligned}
$$

Además

$$
1-|z|^{2}+1-r^{2}<1-|z|^{2} r^{2}+1-|z|^{2} r^{2}<2\left(1-|z|^{2} r^{2}\right)
$$


y

$$
|1-\bar{\zeta} z|+1-r^{2}<|1-\bar{\zeta} z|+1-|z|^{2} r^{2} .
$$

Luego se consigue la estimación

$$
\begin{aligned}
I(z, \zeta) \preceq & \int_{0}^{1} \frac{\left(1-r^{2}\right)^{t_{2}} r}{\left(1-|z|^{2}+1-r^{2}\right)^{1+t_{0}}\left(|1-\bar{\zeta} z|+1-r^{2}\right)^{t_{1}}} d r \\
& +\int_{0}^{1} \frac{\left(1-r^{2}\right)^{t_{2}-t_{1}} r}{\left(|1-\bar{\zeta} z|+1-r^{2}\right)^{1+t_{0}}} d r .
\end{aligned}
$$

Con el cambio de variable $u=1-r^{2}$ la estimación anterior de $I(z, \zeta)$ se escribe de la siguiente forma

$$
I(z, \zeta) \preceq \int_{0}^{1} \frac{u^{t_{2}}}{\left(1-|z|^{2}+u\right)^{1+t_{0}}(|1-\bar{\zeta} z|+u)^{t_{1}}} d u+\int_{0}^{1} \frac{u^{t_{2}-t_{1}}}{(|1-\bar{\zeta} z|+u)^{1+t_{0}}} d u .
$$

Se estima ahora la integral

$$
H_{1}=H_{1}(z, \zeta):=\int_{0}^{1} \frac{u^{t_{2}}}{\left(1-|z|^{2}+u\right)^{1+t_{0}}(|1-\bar{\zeta} z|+u)^{t_{1}}} d u
$$

Se observa que

$$
H_{1}=\int_{0}^{|1-\bar{\zeta} z|} \frac{u^{t_{2}} d u}{\left(1-|z|^{2}+u\right)^{1+t_{0}}(|1-\bar{\zeta} z|+u)^{t_{1}}}+\int_{|1-\bar{\zeta} z|}^{1} \frac{u^{t_{2}} d u}{\left(1-|z|^{2}+u\right)^{1+t_{0}}(|1-\bar{\zeta} z|+u)^{t_{1}}}
$$

Como $u<u+1-|z|^{2}$ y $|1-\bar{\zeta} z|<|1-\bar{\zeta} z|+u$ para la primera integral y para la segunda $u<|1-\bar{\zeta} z|+u \mathrm{y}|1-\bar{\zeta} z|+u<2\left(u+1-|z|^{2}\right)$. Entonces se tiene

$$
\begin{aligned}
H_{1} & \preceq \frac{1}{|1-\bar{\zeta} z|^{t_{1}}} \int_{0}^{|1-\bar{\zeta} z|} u^{t_{2}-1-t_{0}} d u+\int_{|1-\bar{\zeta} z|}^{1}(u+|1-\bar{\zeta} z|)^{t_{2}-t_{0}-t_{1}-1} d u \\
& \preceq \frac{1}{|1-\bar{\zeta} z|^{t_{0}+t_{1}-t_{2}}} .
\end{aligned}
$$

Se estima ahora la integral

$$
H_{2}=H_{2}(z, \zeta):=\int_{0}^{|1-\bar{\zeta} z|} \frac{u^{t_{2}-t_{1}}}{(|1-\bar{\zeta} z|+u)^{1+t_{0}}} d u+\int_{|1-\bar{\zeta} z|}^{1} \frac{u^{t_{2}-t_{1}}}{(|1-\bar{\zeta} z|+u)^{1+t_{0}}} d u .
$$


Como $|1-\bar{\zeta} z|<|1-\bar{\zeta} z|+u$ para la primera integral y $u<|1-\bar{\zeta} z|+u$ para la segunda integral, entonces

$$
\begin{aligned}
H_{2} & \preceq \frac{1}{|1-\bar{\zeta} z|^{1+t_{0}}} \int_{0}^{|1-\bar{\zeta} z|} u^{t_{2}-t_{1}} d u+\int_{|1-\bar{\zeta} z|}^{1}(u+|1-\bar{\zeta} z|)^{t_{2}-t_{0}-t_{1}-1} d u \\
& \preceq \frac{1}{|1-\bar{\zeta} z|^{t_{0}+t_{1}-t_{2}}} .
\end{aligned}
$$

Por lo tanto

$$
I(z, \zeta) \leq H_{1}+H_{2} \leq \frac{C}{|1-\bar{\zeta} z|^{t_{0}+t_{1}-t_{2}}}
$$

\section{2. $\quad$ El operador de Bergman $P_{\beta}$ en $L(p, q, s)={ }_{s} \mathcal{A}_{q}^{p}$}

Se recuerda el siguiente resultado bien conocido, ver Teorema 1.5.5.

Teorema 4.2.1 Supóngase que $-1<q, \beta<\infty$ y $1 \leq p<\infty$. Entonces $P_{\beta}$ es una proyección acotada de $L^{p}\left(\mathbb{D}, d A_{q}\right)$ sobre $\mathcal{A}_{q}^{p}$ si y sólo si $q+1<(\beta+1) p$.

Este resultado puede ser extendido cuando se consideran los espacios pesados ${ }_{s} \mathcal{A}_{q}^{p}=$ $L(p, q, s)$, más precisamente, en esta sección se estudia el operador de Bergman $P_{\beta}$ en los espacios $L(p, q, s)$ cuando $q+1 \geq(\beta+1) p$ para ciertos valores de los parámetros $p, q, s$ у $\beta$.

El caso $p=1$ es tratado por separado.

Teorema 4.2.2 Supóngase que $-1<q<\infty, 0<s<1, p=1$ y $(\beta+1) p=\beta+1=$ $q+1$. Entonces $P_{\beta}=P_{q}$ es un operador acotado de $L^{1}\left(\mathbb{D}, d A_{q}\right)$ en $L(1, q, s)$.

Demostración. Como $p=1$ se tiene que $\beta=q$, así

$$
P_{\beta} f(z)=P_{q} f(z)=\int_{\mathbb{D}} \frac{f(w)}{(1-z \bar{w})^{2+q}} d A_{q}(w) .
$$


Por el Teorema de Fubini se tiene que :

$$
\begin{aligned}
l_{1, q, s}\left(P_{q} f\right)(a) & =\int_{\mathbb{D}}\left|P_{q} f(z)\right|\left(1-\left|\varphi_{a}(z)\right|^{2}\right)^{s} d A_{q}(z) \\
& =\int_{\mathbb{D}}\left|\int_{\mathbb{D}} \frac{f(w)}{(1-z \bar{w})^{2+q}} d A_{q}(w)\right|\left(1-\left|\varphi_{a}(z)\right|^{2}\right)^{s} d A_{q}(z) \\
& \leq \int_{\mathbb{D}} \int_{\mathbb{D}} \frac{\left(1-|w|^{2}\right)^{q}}{|1-z \bar{w}|^{2+q}}|f(w)| d A(w) \frac{\left(1-|z|^{2}\right)^{s}\left(1-|a|^{2}\right)^{s}}{|1-z \bar{a}|^{2 s}} d A_{q}(z) \\
& =\int_{\mathbb{D}}\left(1-|w|^{2}\right)^{q}\left(1-|a|^{2}\right)^{s}|f(w)| \int_{\mathbb{D}} \frac{\left(1-|z|^{2}\right)^{s+q}}{|1-z \bar{w}|^{2+q}|1-z \bar{a}|^{2 s}} d A(z) d A(w) .
\end{aligned}
$$

Escribiendo $t_{0}=q, t_{1}=2 s$ y $t_{2}=s+q$ se obtiene $-1<t_{3}, 0<t_{1}<2+t_{3} \mathrm{y}$ $-1<t_{0}<t_{3}<t_{0}+t_{1}$. Entonces por el Lema 4.1.3 se tiene

$$
\begin{aligned}
l_{1, q, s}\left(P_{q} f\right)(a) & \preceq \int_{\mathbb{D}}\left(1-|w|^{2}\right)^{q}\left(1-|a|^{2}\right)^{s}|f(w)| \frac{1}{|1-a \bar{w}|^{s}} d A(w) \\
& \preceq \int_{\mathbb{D}}\left(1-|w|^{2}\right)^{q}|f(w)| \frac{(1-|a|)^{s}(1+|a|)^{s}}{(1-|a|)^{s}} d A(w) \\
& \preceq \int_{\mathbb{D}}|f(w)| d A_{q}(w) \\
& \preceq\|f\|_{1, q}
\end{aligned}
$$

y la prueba se sigue de esta estimación.

Se sigue la misma idea de la prueba previa para obtener el siguiente resultado.

Teorema 4.2.3 Supóngase que $-1<q<\infty, 0<s<1, p=1$ y $(\beta+1) p=\beta+1<$ $q+1$. Entonces $P_{\beta}$ es un operador acotado de $L^{1}\left(\mathbb{D}, d A_{q}\right)$ en $L(1, q, s)$ si $q<\beta+s$.

Ahora se analiza en caso para $1<p<\infty$ y $(\beta+1) p \leq q+1$.

Del Lema 1.4.2 es inmediato el siguiente resultado.

Lema 4.2.4 Sea $1<p<\infty,-1<q<\infty$ y $\beta \in \mathbb{R}$. Entonces

$$
I_{q, v}(z)=\int_{\mathbb{D}} \frac{\left(1-|w|^{2}\right)^{q}}{|1-z \bar{w}|^{(2+\beta) \cdot \frac{p}{p-1}}} d A(w) \approx h_{v}(z)= \begin{cases}1 & \text { si } v<0 \\ \ln \frac{1}{1-|z|^{2}} & \text { si } v=0 \\ \frac{1}{\left(1-|z|^{2}\right)^{v}} & \text { si } v>0 .\end{cases}
$$

donde

$$
v=v(p, q, \beta)=\frac{(2+\beta) p-(2+q)(p-1)}{p-1} .
$$


Sean $-1<\beta<\infty, 1<p<\infty$ y $0<s<1$. Entonces

$$
\begin{aligned}
I(a) & =\int_{\mathbb{D}}\left|P_{\beta} f(z)\right|^{p}\left(1-\left|\varphi_{a}(z)\right|^{2}\right)^{s} d A_{q}(z) \\
& =\int_{\mathbb{D}}\left|\int_{\mathbb{D}} \frac{f(w)}{(1-z \bar{w})^{2+\beta}} d A_{q}(w)\right|^{p} \frac{\left(1-|z|^{2}\right)^{s}\left(1-|a|^{2}\right)^{s}}{|1-a \bar{z}|^{2 s}} d A_{q}(z) .
\end{aligned}
$$

Se estima la proyección de Bergman usando la desigualdad de Hölder. Así

$$
\begin{aligned}
\left|\int_{\mathbb{D}} \frac{f(w)}{(1-z \bar{w})^{2+\beta}} d A_{q}(w)\right| & \leq\left(\int_{\mathbb{D}}|f(w)|^{p} d A_{q}(w)\right)^{1 / p}\left(\int_{\mathbb{D}} \frac{1}{|1-z \bar{w}|^{(2+\beta) p^{*}}} d A_{q}(w)\right)^{1 / p^{*}} \\
& =\|f\|_{p, q}\left(\int_{\mathbb{D}} \frac{\left(1-|w|^{2}\right)^{q} d A(w)}{|1-z \bar{w}|^{(2+\beta) p^{*}}}\right)^{1 / p^{*}}
\end{aligned}
$$

donde $\frac{1}{p}+\frac{1}{p^{*}}=1$. Con la notación del Lema 4.2.4 se tiene que

$$
I(a) \preceq\|f\|_{p, q}^{p} \int_{\mathbb{D}} h_{v}^{p / p^{*}}(z) \frac{\left(1-|z|^{2}\right)^{s}\left(1-|a|^{2}\right)^{s}}{|1-\bar{a} z|^{2 s}} d A_{q}(z) .
$$

Se estima la última integral aplicando de nuevo el Lema 1.4.2 y el Lema 4.2.4, en particular cada caso se origina a partir del signo de (4.4).

Teorema 4.2.5 Sean $-1<q<\infty, \frac{q+2}{q+1}<p<\infty, 0<s<1 y-1<\beta<$ $\frac{q(p-1)-2}{p}$. Entonces

$$
P_{\beta}: L^{p}\left(\mathbb{D}, d A_{q}\right) \mapsto L(p, q, s)
$$

es un operador acotado si $v=\frac{(2+\beta) p-(2+q)(p-1)}{p-1} \leq 0$.

Demostración. Si $v \leq 0$, por los Lemas 4.2.4 y 1.4.2 se tiene

$$
I(a) \preceq\|f\|_{p, q}^{p}
$$

y la prueba se sigue de esta afirmación.

Se estudia ahora la integral 4.5 cuando $v>0$, esto es

$$
\int_{\mathbb{D}} \frac{1}{\left(1-|z|^{2}\right)^{(2+\beta) p-(2+q)(p-1)}} \cdot \frac{\left(1-|z|^{2}\right)^{s+q}}{|1-z \bar{a}|^{2 s}} d A(z)=\int_{\mathbb{D}} \frac{\left(1-|z|^{2}\right)^{p(q-\beta)+s-2}}{|1-z \bar{a}|^{2 s}} d A(z) .
$$

Estimamos esta integral aplicando el Lema 1.4.2. Entonces es necesario tener $p(q-$ $\beta)+s-2>-1$ y esto es equivalente a

$$
\beta<\frac{p q+s-1}{p}
$$

y así 
Lema 4.2.6 Sean $1<p<\infty,-1<q<\infty, \beta \in \mathbb{R}$ y $p(q-\beta)+s-2>-1$. Entonces

$$
I_{\gamma}(z)=\int_{\mathbb{D}} \frac{\left(1-|z|^{2}\right)^{p(q-\beta)+s-2}}{|1-z \bar{a}|^{2 s}} d A(z) \approx h_{\gamma}(z)= \begin{cases}1 & \text { si } \gamma<0 \\ \ln \frac{1}{1-|z|^{2}} & \text { si } \gamma=0 \\ \frac{1}{\left(1-|z|^{2}\right)^{\gamma}} & \text { si } \gamma>0\end{cases}
$$

donde $\gamma=\gamma(p, q, s)=p(\beta-q)+s$.

Así se necesitan estudiar los tres casos asociados a $\gamma$. Se considera primero el caso cuando $\gamma>0$ y es formulado en el siguiente teorema, pero antes se necesita el siguiente resultado.

Lema 4.2.7 Sean $1<p<\infty,-1<q<\infty y \frac{1}{2}<s<1$. Sean

$$
a=\operatorname{máx}\left\{-1, \frac{q(p-1)-2}{p}, \frac{p q-s}{p}\right\} \quad y \quad b=\min \left\{\frac{q+1-p}{p}, \frac{p q+s-1}{p}\right\} \text {. }
$$

Entonces el intervalo $(a, b)$ es no vacío si y sólo si

$$
\frac{1-p-s}{p}<q<\frac{1-p+s}{p-1}
$$

Además

$$
a=\left\{\begin{array}{lll}
-1 & \text { si } & q<\frac{s-p}{p} \\
\frac{p q-s}{p} & \text { si } & q \geq \frac{s-p}{p}
\end{array}\right.
$$

$y$

$$
b=\left\{\begin{array}{lll}
\frac{q+1-p}{p} & \text { si } & q>\frac{2-p-s}{p-1} \\
\frac{p q+s-1}{p} & \text { si } & q \leq \frac{2-p-s}{p-1}
\end{array}\right.
$$

Teorema 4.2.8 Sean $1<p<\infty, \frac{1}{2}<s<1, \frac{1-p-s}{p}<q<\frac{1-p+s}{p-1}, a<\beta<b$, con a y $b$ como en el lema previo y $v>0$ (ver Lema 4.2.4). Entonces

$$
P_{\beta}: L^{p}\left(\mathbb{D}, d A_{q}\right) \mapsto L(p, q, s)
$$

es un operador acotado. Además

$$
I(a) \preceq\left(1-|a|^{2}\right)^{p(q-\beta)}\|f\|_{p, q}^{p} .
$$


Demostración. Por hipótesis $-1<\beta<\frac{q+1-p}{p}$. Como $v>0$, entonces $\beta>$ $\frac{q(p-1)-2}{p}$ y por $(4.6)$, se tiene

$$
I(a) \preceq C\left(1-|a|^{2}\right)^{s}\|f\|_{p, q}^{p} \int_{\mathbb{D}} \frac{\left(1-|z|^{2}\right)^{p(q-\beta)+s-2}}{|1-z \bar{a}|^{2 s}} d A(z) .
$$

Por hipótesis $\beta<\frac{p q+s-1}{p} \mathrm{y} \gamma=p(\beta-q)+s>0$, y esto es equivalente a $\frac{p q-s}{p}<\beta$. Entonces por el Lema 4.2.6 se tiene

$$
\begin{aligned}
I(a) & \preceq\left(1-|a|^{2}\right)^{s}\|f\|_{p, q}^{p} \frac{1}{\left(1-|a|^{2}\right)^{p(\beta-q)+s}} \\
& \preceq\left(1-|a|^{2}\right)^{p(q-\beta)}\|f\|_{p, q}^{p}
\end{aligned}
$$

y por hipótesis $\beta<q$ y así se concluye la prueba.

Se describe explícitamente al intervalo $(a, b)$ del teorema previo.

Proposición 4.2.9 Con las hipótesis del Lema 4.2.7 se tiene que

i. $(a, b)=\left(-1, \frac{q+1-p}{p}\right)$ si y sólo si $\frac{s}{2 s-1}<p<\infty$ y $\frac{2-p-s}{p-1}<q<$ $\frac{s-p}{p}$.

ii. $(a, b)=\left(-1, \frac{p q+s-1}{p}\right)$ si y sólo si

ii. $11<p<\frac{s}{2 s-1}$ y $\frac{1-p-s}{p}<q<\frac{s-p}{p}$ ó

ii. $2 \frac{s}{2 s-1} \leq p \quad y \quad \frac{1-p-s}{p}<q<\frac{2-p-s}{p-1}$.

iii. $(a, b)=\left(\frac{p q-s}{p}, \frac{q+1-p}{p}\right)$, con

iii. $1<p<\frac{s}{2 s-1} \quad y \frac{2-p-s}{p-1}<q<\frac{1-p+s}{p-1}$ ó

iii. $2 \frac{s}{2 s-1} \leq p \quad y \quad \frac{s-p}{p}<q<\frac{1-p+s}{p-1}$. 


$$
\begin{aligned}
& \text { iv. }(a, b)=\left(\frac{p q-s}{p}, \frac{p q+s-1}{p}\right) \text { si y sólo si } 1<p<\frac{s}{2 s-1} \text { y } \frac{s-p}{p}<q< \\
& \frac{2-p-s}{p-1} \text {. }
\end{aligned}
$$

Demostración. Para mostrar el caso $i$ nótese que

$$
-1<\beta<\frac{q+1-p}{p}
$$

si y sólo si

$$
q<\frac{s-p}{p} ; \quad q>\frac{2-p-s}{p-1} ; \quad \text { y } \quad \frac{1-p-s}{p}<q<\frac{1-p+s}{p-1},
$$

esto es, $q$ debe satisfacer

$$
\text { máx }\left\{\frac{2-p-s}{p-1}, \frac{1-p-s}{p}\right\}<q<\operatorname{mín}\left\{\frac{s-p}{p}, \frac{1-p+s}{p-1}\right\},
$$

ó equivalentemente

$$
\begin{aligned}
& \frac{2-p-s}{p-1}<\frac{s-p}{p} \quad \Leftrightarrow p>\frac{2 s}{2 s-1} \\
& \frac{2-p-s}{p-1}<\frac{1-p+s}{p-1} \Leftrightarrow \frac{1}{2}<s \\
& \frac{1-p-s}{p}<\frac{s-p}{p} \Leftrightarrow \frac{1}{2}<s \\
& \frac{1-p-s}{p}<\frac{1-p+s}{p-1} \Leftrightarrow p>\frac{s-1}{2 s-1} .
\end{aligned}
$$

Puesto que $\frac{1}{2}<s<1$ entonces

$$
\operatorname{máx}\left\{\frac{2-p-s}{p-1}, \frac{1-p-s}{p}\right\}=\frac{2-p-s}{p-1},
$$

y

$$
\min \left\{\frac{1-p+s}{p-1}, \frac{s-p}{p}\right\}=\frac{s-p}{p} .
$$

Esto prueba la afirmación. Los demas casos son análogos.

Se considera ahora el caso cuando $\gamma<0$, el cual es dividido en dos casos: $1<p \leq 2$ y $2<p<\infty$. En el siguiente teorema el resultado es formulado para $1<p \leq 2$ y se necesita el siguiente resultado. 
Lema 4.2.10 Sean $1<p \leq 2,-1<q<\infty$ y $0<s<1$. Sean

$$
a=\operatorname{máx}\left\{-1, \frac{q(p-1)-2}{p}\right\} \quad y \quad b=\operatorname{mín}\left\{\frac{q+1-p}{p}, \frac{p q+s-1}{p}, \frac{p q-s}{p}\right\} \text {. }
$$

Entonces el intervalo $(a, b)$ es no vacío si y sólo si

1. Para $0<s \leq \frac{1}{2}$ se tiene $\frac{1-p-s}{p}<q<\infty$. Además

$$
a=\left\{\begin{array}{lll}
-1 & \text { si } & q<\frac{2-p}{p-1} \\
\frac{q(p-1)-2}{p} & \text { si } & q \geq \frac{2-p}{p-1}
\end{array}\right.
$$

$y$

$$
b=\left\{\begin{array}{lll}
\frac{q+1-p}{p} & \text { si } & q>\frac{2-p-s}{p-1} \\
\frac{p q+s-1}{p} & \text { si } & q \leq \frac{2-p-s}{p-1}
\end{array}\right.
$$

2. Para $\frac{1}{2}<s<1$ se tiene $\frac{s-p}{p}<q<\infty$. Además

$$
a= \begin{cases}-1 & \text { si } \quad q<\frac{2-p}{p-1} \\ \frac{q(p-1)-2}{p} & \text { si } q \geq \frac{2-p}{p-1}\end{cases}
$$

$y$

$$
b=\left\{\begin{array}{lll}
\frac{q+1-p}{p} & \text { si } & q>\frac{1-p+s}{p-1} \\
\frac{p q-s}{p} & \text { si } & q \leq \frac{1-p+s}{p-1}
\end{array}\right.
$$

Teorema 4.2.11 Sean $1<p \leq 2,0<s<1$, $\operatorname{máx}\left\{\frac{1-p-s}{p}, \frac{s-p}{p}\right\}<q<\infty y$ $a<\beta<b$, con a y $b$ como en el Lema previo. Entonces

$$
P_{\beta}: L^{p}\left(\mathbb{D}, d A_{q}\right) \mapsto L(p, q, s)
$$

es un operador acotado. Además

$$
I(a) \preceq\left(1-|a|^{2}\right)^{s}\|f\|_{p, q}^{p}
$$


Demostración. Obsérvese que

$$
\operatorname{máx}\left\{\frac{1-p-s}{p}, \frac{s-p}{p}\right\}=\left\{\begin{array}{lll}
\frac{1-p-s}{p} & \text { si } \quad 0<s<\frac{1}{2} \\
\frac{s-p}{p} & \text { si } \frac{1}{2} \leq s<1
\end{array}\right.
$$

y el resultado se obtiene imitando la prueba del Teorema 4.2.8.

Se decribe explícitamente al intervalo $(a, b)$ del teorema previo.

\section{Proposición 4.2.12}

I. Para $1<p \leq 2,0<s \leq \frac{1}{2}, \frac{1-p-s}{p}<q<\infty$ y a, b como en 1 del Lema 4.2.10, se tiene que

i. $(a, b)=\left(-1, \frac{q+1-p}{p}\right)$ si y sólo si $\frac{2-p-s}{p-1}<q<\frac{2-p}{p-1}$.

ii. $(a, b)=\left(-1, \frac{p q+s-1}{p}\right)$ si y sólo si $\frac{1-p-s}{p}<q<\frac{2-p-s}{p-1}$.

iii. $(a, b)=\left(\frac{q(p-1)-2}{p}, \frac{q+1-p}{p}\right)$ si y sólo si $\frac{2-p}{p-1}<q<\infty$.

$i v .(a, b)=\left(\frac{q(p-1)-2}{p}, \frac{p q+s-1}{p}\right)=\emptyset$.

II. Para $1<p \leq 2, \frac{1}{2}<s<1, \frac{s-p}{p}<q<\infty$ y a, b como en 2 del Lema 4.2.10, se tiene que

i. $(a, b)=\left(-1, \frac{q+1-p}{p}\right)$ si y sólo si $\frac{1-p+s}{p-1}<q<\frac{2-p}{p-1}$.

ii. $(a, b)=\left(-1, \frac{p q-s}{p}\right)$ si y sólo si $\frac{s-p}{p}<q<\frac{1-p+s}{p-1}$.

iii. $(a, b)=\left(\frac{q(p-1)-2}{p}, \frac{q+1-p}{p}\right)$ si y sólo si $\frac{2-p}{p-1}<q<\infty$.

$i v . \quad(a, b)=\left(\frac{q(p-1)-2}{p}, \frac{p q-s}{p}\right)=\emptyset$.

Ahora el resultado es formulado para $\gamma<0$ y $2<p<\infty$ y se necesitará el siguiente resultado. 
Lema 4.2.13 Sean $2<p<\infty,-1<q<\infty$ y $0<s<1$. Sean

$$
a=\operatorname{máx}\left\{-1, \frac{q(p-1)-2}{p}\right\} \quad y \quad b=\min \left\{\frac{q+1-p}{p}, \frac{p q+s-1}{p}, \frac{p q-s}{p}\right\} \text {. }
$$

Entonces el intervalo $(a, b)$ es no vacío si y sólo si

1. Para $0<s \leq \frac{1}{2}$ se tiene que $\frac{1-p-s}{p}<q<\frac{3-p}{p-2}$. Además

$$
a= \begin{cases}-1 & \text { si } \quad q<\frac{2-p}{p-1} \\ \frac{q(p-1)-2}{p} & \text { si } q \geq \frac{2-p}{p-1}\end{cases}
$$

$y$

$$
b=\left\{\begin{array}{lll}
\frac{q+1-p}{p} & \text { si } & q>\frac{2-p-s}{p-1} \\
\frac{p q+s-1}{p} & \text { si } & q \leq \frac{2-p-s}{p-1}
\end{array}\right.
$$

2. Para $\frac{1}{2}<s<1$ se tiene $\frac{s-p}{p}<q<\frac{3-p}{p-2}$. Además

$$
a= \begin{cases}-1 & \text { si } q<\frac{2-p}{p-1} \\ \frac{q(p-1)-2}{p} & \text { si } q \geq \frac{2-p}{p-1}\end{cases}
$$

$y$

$$
b=\left\{\begin{array}{lll}
\frac{q+1-p}{p} & \text { si } & q>\frac{1-p+s}{p-1} \\
\frac{p q-s}{p} & \text { si } & q \leq \frac{1-p+s}{p-1}
\end{array}\right. \text {. }
$$

Teorema 4.2.14 Sean $2<p<\infty, 0<s<1$, máx $\left\{\frac{1-p-s}{p}, \frac{s-p}{p}\right\}<q<$ $\frac{3-p}{p-2}$ y $a<\beta<b$, con $a$ y $b$ como en el Lema 4.2.13. Entonces

$$
P_{\beta}: L^{p}\left(\mathbb{D}, d A_{q}\right) \mapsto L(p, q, s)
$$

es un operador acotado. Además

$$
I(a) \preceq\left(1-|a|^{2}\right)^{s}\|f\|_{p, q}^{p} .
$$


Demostración. La prueba es similar a la hecha en el Teorema 4.2.8.

Se describen explícitamente los intervalos $(a, b)$ del teorema previo.

\section{Proposición 4.2.15}

I. Para $2<p<\infty, 0<s \leq \frac{1}{2}, \frac{1-p-s}{p}<q<\frac{3-p}{p-2}$ y a, b como en 1 del Lema 4.2.13 se tiene que

i. $(a, b)=\left(-1, \frac{q+1-p}{p}\right)$ si y sólo si $\frac{2-p-s}{p-1}<q<\frac{2-p}{p-1}$.

ii. $(a, b)=\left(-1, \frac{p q+s-1}{p}\right)$ si y sólo si $\frac{1-p-s}{p}<q<\frac{2-p-s}{p-1}$.

iii. $(a, b)=\left(\frac{q(p-1)-2}{p}, \frac{q+1-p}{p}\right)$ si y sólo si $\frac{2-p}{p-1}<q<\frac{3-p}{p-2}$.

$i v .(a, b)=\left(\frac{q(p-1)-2}{p}, \frac{p q+s-1}{p}\right)=\emptyset$.

II. Para $2<p<\infty, \frac{1}{2}<s<1, \frac{s-p}{p}<q<\frac{3-p}{p-2}$ y a, b como en 2 del Lema 4.2.13 se tiene que

i. $(a, b)=\left(-1, \frac{q+1-p}{p}\right)$ si y sólo si $\frac{1-p+s}{p-1}<q<\frac{2-p}{p-1}$.

ii. $(a, b)=\left(-1, \frac{p q-s}{p}\right)$ si y sólo si $\frac{s-p}{p}<q<\frac{1-p+s}{p-1}$.

iii. $(a, b)=\left(\frac{q(p-1)-2}{p}, \frac{q+1-p}{p}\right)$ si y sólo si $\frac{2-p}{p-1}<q<\frac{3-p}{p-2}$.

$i v .(a, b)=\left(\frac{q(p-1)-2}{p}, \frac{p q-s}{p}\right)=\emptyset$.

Se considera ahora el caso $\gamma=0$. El resultado se formula en el siguiente teorema, pero antes se necesita el siguiente resultado que es directo.

Lema 4.2.16 Sean $1<p<\infty,-1<q<\infty y \frac{1}{2}<s<1$. Sea $a=\operatorname{máx}\left\{-1, \frac{q(p-1)-2}{p}\right\}, \quad b=\operatorname{mín}\left\{\frac{q+1-p}{p}, \frac{p q+s-1}{p}\right\} \quad y \quad \beta=\frac{p q-s}{p}$. 
Entonces $a<\beta<b$ si y sólo si

$$
\frac{s-p}{p}<q<\frac{1-p+s}{p-1} .
$$

Además

$$
a=\left\{\begin{array}{lll}
-1 & \text { si } & q<\frac{2-p}{p-1} \\
\frac{q(p-1)-2}{p} & \text { si } & q \geq \frac{2-p}{p-1}
\end{array}\right.
$$

$y$

$$
b=\left\{\begin{array}{lll}
\frac{q+1-p}{p} & \text { si } & q<\frac{2-p-s}{p-1} \\
\frac{p q+s-1}{p} & \text { si } & q \geq \frac{2-p-s}{p-1}
\end{array}\right.
$$

Teorema 4.2.17 Sean $1<p<\infty, \frac{1}{2}<s<1, \frac{s-p}{p}<q<\frac{1-p+s}{p-1}$ y $a<\beta<b$, con $a, b$ y $\beta=\frac{p q-s}{p}$ como en el lema 4.2.16. Entonces

$$
P_{\beta}: L^{p}\left(\mathbb{D}, d A_{q}\right) \mapsto L(p, q, s)
$$

es un operador acotado. Además

$$
I(a) \preceq C\left(1-|a|^{2}\right)^{s}\|f\|_{p, q}^{p} .
$$

Demostración. La demostración es similar a la del Teorema 4.2.11.

Nuevamente, se pueden describir explícitamente los intervalos $(a, b)$ del lema previo.

Proposición 4.2.18 Sean $1<p<\infty, \frac{1}{2}<s<1, \frac{s-p}{p}<q<\frac{1-p+s}{p-1}$ y $a, b$ como en el Lema 4.2.16. Entonces

i. $(a, b)=\left(-1, \frac{q+1-p}{p}\right)$ si y sólo si $\frac{s-p}{p}<q<\frac{2-p-s}{p-1}$.

ii. $(a, b)=\left(-1, \frac{p q+s-1}{p}\right)$ si y sólo si

$$
\text { ii. } 11<p<\frac{s}{2 s-1} y \frac{2-p-s}{p-1}<q<\frac{1-p+s}{p-1} \text { ó }
$$




$$
\text { ii.2 } \frac{s}{2 s-1}<p<\infty y \frac{s-p}{p}<q<\frac{1-p+s}{p-1}
$$

iii. $(a, b)=\left(\frac{q(p-1)-2}{p}, \frac{q+1-p}{p}\right)=\emptyset$

$i v .(a, b)=\left(\frac{q(p-1)-2}{p}, \frac{p q+s-1}{p}\right)=\emptyset$. 


\section{Conclusiones y Perspectivas}

En el presente trabajo se han obtenido los siguientes resultados:

1. Se establecen relaciones explícitas entre las escalas de los espacios de Bergman pesados ${ }_{s} \mathcal{A}_{q}^{p}$ y las escalas de los espacios de Bloch.

2. Se prueba que los espacios de Bergman pesados son invariantes bajo transformaciones de Möbius, más aún el operador composición con éstas es acotado.

3. Para $0<s<1$, los espacios ${ }_{s} \mathcal{A}_{q}^{p}$ son una escala de espacios con inclusiones estrictas .

4. Se establecen vínculos con los espacios $F(p, q, s)$ estudiados por Zhao et al.: algunas caracterizaciones y propiedades relacionadas con los espacios estudiados en este trabajo.

5. Se estudia el operador de Bergman $P_{\beta}$ del espacio $L^{p}\left(\mathbb{D}, d A_{q}\right)$ en los espacios de Bergman pesados ${ }_{s} \mathcal{A}_{q}^{p}=L(p, q, s)$ y se prueba que es un operador acotado para ciertos valores de $\beta, p, q$ y $s$ que en particular satisfacen la relación $q+1 \geq$ $(\beta+1) p$ y así el Teorema 1.5.5 se puede extender gracias a la adición del peso $1-\left|\varphi_{z}(w)\right|^{2}$.

En este trabajo se logran establecer vínculos importantes entre diferentes espacios de funciones clásicos.

La perspectiva general de este trabajo se basa en el hecho de que los espacios de Bergman definidos sobre la bola unitaria de $\mathbb{C}^{n}$ han sido estudiados en forma profusa en los últimos años, por ello la continuación inmediata de este trabajo es: 
Formular y estudiar la teoría de espacios de Bergman pesados para funciones holomorfas $f: B \rightarrow \mathbb{C}$ donde $B \subset \mathbb{C}^{n}$ es la bola unitaria. Para ello se consideran los automorfismos de la bola unitaria $\varphi_{a}: B \rightarrow B$ dados por:

$$
\varphi_{a}(z)=\frac{a-P_{a}(z)-s_{a} Q_{a}(z)}{1-\langle z, a\rangle}, \quad z \in B .
$$

donde $s_{a}=\sqrt{1-|a|^{2}}, P_{a}$ es la proyección normal ortogonal de $\mathbb{C}^{n}$ sobre el subespacio unidimensional generado por $a$ y $Q_{a}$ es la proyección ortogonal de $\mathbb{C}^{n}$ sobre $\mathbb{C}^{n}$ menos el subespacio generado por $a$. Es claro que

$$
P_{a}(z)=\frac{\langle z, a\rangle}{|a|^{2}} a, \quad z \in \mathbb{C}^{n},
$$

y

$$
Q_{a}(z)=z-\frac{\langle z, a\rangle}{|a|^{2}} a, \quad z \in B .
$$

Cuando $a=0$ simplemente se define $\varphi_{a}(z)=-z$.

La función invariante de Green esá dada por:

$$
G(z)=\frac{1}{2 \pi} \int_{|z|}^{1} \frac{\left(1-t^{2}\right)^{n-1}}{t^{2 n-1}} d t, \quad z \in B
$$

La medida $d A_{\alpha}$ se define por

$$
d A_{\alpha}(z)=c_{\alpha}\left(1-|z|^{2}\right)^{\alpha} d A(z),
$$

donde $c_{\alpha}$ es una constante de normalización, tal que $d A_{\alpha}(B)=1$. Se tendrá un caso de especial interés cuando $\alpha=-(n+1)$, en este caso se denota a la medida resultante por

$$
d \tau(z)=\frac{d A(z)}{\left(1-|z|^{2}\right)^{n+1}},
$$

y se conoce como la medida invariante en $B$. 


\section{Apéndice}

Teorema 1 Sean $0<p<\infty,-2<q<\infty$ y $f \in \mathcal{H}$. Supóngase que $l_{p, q, s}(f)(0)<\infty$, entonces:

a) Para $0<s<\infty$, se tiene

$$
\int_{\mathbb{D}}|f(w)|^{p} \ln ^{s} \frac{1}{|w|} d A_{q}(w) \leq t \int_{\mathbb{D}}|f(w)|^{p}\left(1-|w|^{2}\right)^{s} d A_{q}(w),
$$

donde $t=t(q, s, R)$ para algún $0<R<1$ fijo.

b) Para $0<s<1$, se tiene

$$
\int_{\mathbb{D}}|f(w)|^{p}|w|^{-2 s}\left(1-|w|^{2}\right)^{s} d A_{q}(w) \leq \tilde{t} \int_{\mathbb{D}}|f(w)|^{p}\left(1-|w|^{2}\right)^{s} d A_{q}(w)
$$

donde $\tilde{t}=\tilde{t}(q, s, R)$ para algún $0<R<1$ fijo.

Demostración. a) Se sigue de la idea de la prueba dada en el Teorema 2.2 of Aulaskari et al [AuStXi] con todos sus detalles. Sea $c=.0183403$ la raiz de $-\ln x=4\left(1-x^{2}\right)$. Sea $c<R<1$ fijo. Se define

$$
\begin{aligned}
0<\frac{1}{\tau(q, s, R)}=\int_{c}^{R}\left(1-r^{2}\right)^{q+s} r d r & =\frac{1}{2(1+q+s)}\left(\left(1-c^{2}\right)^{1+q+s}-\left(1-R^{2}\right)^{1+q+s}\right) \\
& =\frac{1}{2(1+q+s)}\left(, 999664^{1+q+s}-\left(1-R^{2}\right)^{1+q+s}\right) .
\end{aligned}
$$

Como $|f|^{p}$ es subarmonica

$$
\begin{aligned}
\frac{1}{\tau(q, s, R)} \int_{0}^{2 \pi}\left|f\left(c e^{i \theta}\right)\right|^{p} d \theta & =\int_{c}^{R}\left(1-r^{2}\right)^{q+s} r d r \int_{0}^{2 \pi}\left|f\left(c e^{i \theta}\right)\right|^{p} d \theta \\
& \leq \int_{c}^{R}\left(1-r^{2}\right)^{q+s} r d r \int_{0}^{2 \pi}\left|f\left(r e^{i \theta}\right)\right|^{p} d \theta \\
& =\int_{\mathbb{D}_{R}-\mathbb{D}_{c}}|f(w)|^{p}\left(1-|w|^{2}\right)^{s} d A_{q}(w) .
\end{aligned}
$$


Por lo tanto

$$
\int_{0}^{2 \pi}\left|f\left(c e^{i \theta}\right)\right|^{p} d \theta \leq \tau(q, s, R) \int_{\mathbb{D}_{R}}|f(w)|^{p}\left(1-|w|^{2}\right)^{s} d A_{q}(w) .
$$

Se define

$$
0<\tilde{\tau}(q, s)=\int_{0}^{c} r\left(1-r^{2}\right)^{q} \ln ^{s} \frac{1}{r} d r
$$

Por subarmonicidad y (3), se tiene la estimación

$$
\begin{aligned}
\int_{\mathbb{D}_{c}}|f(w)|^{p}\left(1-|w|^{2}\right)^{q} \ln ^{s} \frac{1}{|w|} d A(w) & =\int_{0}^{c} \int_{0}^{2 \pi}\left|f\left(r e^{i \theta}\right)\right|^{p} r\left(1-r^{2}\right)^{q} \ln ^{s} \frac{1}{r} d \theta d r \\
& \leq \int_{0}^{c} \int_{0}^{2 \pi}\left|f\left(c e^{i \theta}\right)\right|^{p} r\left(1-r^{2}\right)^{q} \ln ^{s} \frac{1}{r} d \theta d r \\
& =\int_{0}^{c} r\left(1-r^{2}\right)^{q} \ln ^{s} \frac{1}{r} d r \int_{0}^{2 \pi}\left|f\left(c e^{i \theta}\right)\right|^{p} d \theta \\
& \leq \tau(q, s, R) \tilde{\tau}(q, s) \int_{\mathbb{D}_{R}}|f(w)|^{p}\left(1-|w|^{2}\right)^{s} d A_{q}(w) \\
& \leq \tau(q, s, R) \tilde{\tau}(q, s) \int_{\mathbb{D}}|f(w)|^{p}\left(1-|w|^{2}\right)^{s} d A_{q}(w)
\end{aligned}
$$

De la desigualdad

$$
-\ln x \leq 4\left(1-x^{2}\right) \quad \text { for each } x \in(c, 1]
$$

se tiene que

$$
\begin{aligned}
\int_{\mathbb{D}-\mathbb{D}_{c}}|f(w)|^{p}\left(1-|w|^{2}\right)^{q} \ln ^{s} \frac{1}{|w|} d A(w) & \leq 4^{s} \int_{\mathbb{D}-\mathbb{D}_{c}}|f(w)|^{p}\left(1-|w|^{2}\right)^{s} d A_{q}(w) \\
& \leq 4^{s} \int_{\mathbb{D}}|f(w)|^{p}\left(1-|w|^{2}\right)^{s} d A_{q}(w) .
\end{aligned}
$$

Sea $t(q, s, R)=\tau(q, s, R) \tilde{\tau}(q, s)+4^{s}$. Combinando (5) y (7), se tiene

$$
\int_{\mathbb{D}}|f(w)|^{p}\left(1-|w|^{2}\right)^{q} \ln ^{s} \frac{1}{|w|} d A(w) \leq t(q, s, R) \int_{\mathbb{D}}|f(w)|^{p}\left(1-|w|^{2}\right)^{s} d A_{q}(w) .
$$

b) Para $0<s<1$, se considera en lugar de (4), la siguiente igualdad

$$
0<\int_{0}^{c} r^{1-2 s}\left(1-r^{2}\right)^{q+s} d r=\frac{1}{2} B\left[c^{2}, 1-s, 1+q+s\right]
$$


donde $B$ denota a la función incompleta Beta. Entonces se muestra la fórmula (2) por un camino similar a (1).

Teorema 2 Sean $0<p<\infty,-2<q<\infty$ y $0 \leq s<\infty$. Entonces ${ }_{s} \mathcal{A}_{q}^{p}=L(p, q, s)$.

Demostración. Se tiene que

$$
1-x^{2} \leq-2 \ln x \quad \text { para cada } x \in(0,1] .
$$

Tomando $x=\varphi_{z}(w)$ se tiene $1-\left|\varphi_{z}(w)\right|^{2} \leq 2 g(w, z)$. Por lo tanto

$$
l_{p, q, s}(f)(z) \leq 2 h_{p, q, s}(f)(z) \quad \text { para cada } z \in \mathbb{D} .
$$

Así ${ }_{s} \mathcal{A}_{q}^{p} \subset L(p, q, s)$.

Se muestra ahora que $L(p, q, s) \subset{ }_{s} \mathcal{A}_{q}^{p}$. Para esto, sea $f \in L(p, q, s)$, entonces $l_{p, q, s}(f)(z)<\infty$. Por hipótesis y el Lema 3.1.10, $l_{p, q, s}(f \circ \varphi)(0)<\infty$. Puesto que $\left|f \circ \varphi_{a}\right|^{p}$ es subarmonica, la fórmula (1) se escribe

$$
\left.\int_{\mathbb{D}}\left|f\left(\varphi_{z}(w)\right) \ln ^{s} \frac{1}{|w|} d A_{q}(w) \leq t(q, s, R) \int_{\mathbb{D}}\right| f\left(\varphi_{z}(w)\right)\right|^{p}\left(1-|w|^{2}\right)^{s} d A_{q}(w) .
$$

Considerando el cambio de variable $w=\varphi_{z}(v)$ para obtener

$$
\begin{aligned}
\int_{\mathbb{D}}|f(v)|^{p}\left|\varphi_{z}^{\prime}(v)\right|^{2}\left(1-\left|\varphi_{z}(v)\right|^{2}\right)^{q} \ln ^{s} \frac{1}{\left|\varphi_{z}(v)\right|} d A(v) \\
\leq t(q, s, R) \int_{\mathbb{D}}|f(v)|^{p}\left|\varphi_{z}^{\prime}(v)\right|^{2}\left(1-\left|\varphi_{z}(v)\right|^{2}\right)^{q+s} d A(v)
\end{aligned}
$$

o, equivalentemente,

$$
\begin{aligned}
0 & \leq \int_{\mathbb{D}}|f(v)|^{p}\left|\varphi_{z}^{\prime}(v)\right|^{2}\left(1-\left|\varphi_{z}(v)\right|^{2}\right)^{q}\left(t(q, s, R)\left(1-\left|\varphi_{z}(v)\right|^{2}\right)^{s}-\ln ^{s} \frac{1}{\left|\varphi_{z}(v)\right|}\right) d A(v) \\
& \leq \rho(z,|q|+2) \int_{\mathbb{D}}|f(v)|^{p}\left(1-|v|^{2}\right)^{q}\left(t(q, s, R)\left(1-\left|\varphi_{z}(v)\right|^{2}\right)^{s}-\ln ^{s} \frac{1}{\left|\varphi_{z}(v)\right|}\right) d A(v)
\end{aligned}
$$

entonces se obtiene que

$$
\begin{aligned}
& \int_{\mathbb{D}}|f(v)|^{p} \ln ^{s} \frac{1}{\left|\varphi_{z}(v)\right|} d A_{q}(v) \\
& \quad \leq t(q, s, R) \int_{\mathbb{D}}|f(v)|^{p}\left(1-\left|\varphi_{z}(v)\right|^{2}\right)^{s} d A_{q}(v),
\end{aligned}
$$

y de aquí se obtiene lo deseado. 


\section{Bibliografía}

[AuStXi] Aulaskari, R.; D. Stegenga; Xiao, J. Some subclasses of BMOA and their characterization in terms of Carleson measures. Rocky Mountain J. Math. 26, (1996), Pp. 485-506.

[AuXiZh] Aulaskari, R.; Xiao, J.; Zhao, R. On subspaces and subsets of BMOA and UBC. Analysis, Vol. 15. 1995. Pp. 101-102.

[Bae] Baernstein, A. Analytic Functions of Bounded Mean Oscillation, Washington University, St. Louis. USA

[CaRe] Carmona L. Luis Javier and Reséndis O. L.F. A direct proof of $V M O_{\delta} \cap$ $H(\mathbb{D})=\mathcal{B}_{0}$. Aportaciones Matemáticas Memorias 50 (pp. 3-14). Artículo de Investigación, (2016).

[CaReTo] Carmona L. Luis Javier, Reséndis O. L.F. and Tovar S. Luis M., A. Weighted Bergman Spaces, Hypercomplex Analysis: New Perspectives and Applications, Trends in Mathematics, Ed. Springer International Publishing Switzerland, 2014, p. $89-110$.

[Duren] Duren, P. And Schuster, A., Bergman Spaces, AMS, Providence, 2004.

[Ga] Garnett, John B. Bounded Analytic Functions Pure and Applied Mathematics 96, p.467, Academic Press, Inc., New York-London (1981)

[HeSt] E. Hewitt, and K. Stromberg Real and Abstract Analysis, Springer-Verlag (1975).

[HeKoZh] Hedenmalm, H., Korenblum, B., And Zhu, K., Theory of Bergman Spaces, Springer, New York, 2000.

[HuipingLi] Li, H. Hankel operators on the Bergman space of multiply-connected domains, J. Operator Theory 28 (1992), 321-335. 
[MaPa] M. Mateljevic, M. Pavlovic, $L^{p}$ behaviour of power series with positive coefficients and Hardy spaces, Proc. Amer. Math. Soc. 87, (1983), 309-316.

[OrFa] Ortega M. and Fabrega J, Pointwise Multipliers and Corona type Theorem, Ann. Inst. Fourier, Grenoble, Tomo 46, No. 1 (1996), p.111-137.

[OrFa1] J.M. Ortega and J. Fábrega , Corona Type Decomposition in some Besov spaces, Math. Scand. 78 (1996), p.93-111.

[PeReTo] Pérez H. Jorge, Reséndis O. L.F. and Tovar S. Luis M., A.Some hyperbolic classes of analytic functions in the unit disk, Boletín de la Sociedad Matemática Mexicana, October 2015, Volume 21, Issue 2, pp 171-188.

[Ra] J. Rättyä, On some complex Function Spaces and Classes, Ann. Acad. Scie. Fenn. Math. Diss. 124, (2001), 1-73.

[Zha] R. Zhao, On $\alpha$-Bloch functions and VMOA., Acta Mathematica Scientia, 16 (3), p. 349-360, (1996)

[KeZh] Zhu, K. VMO, ESV and Toeplitz operators on the Bergman spaces, Trans. Amer. Math. Soc. 309 (1987), 617-646.

[Zhu1] Zhu, K. Spaces of holomorphic functions in the unit ball, Graduate Texts in Mathematics, Springer, USA, 2005.

[Zhu2] Zhu, K., Operator Theory in Funtions Spaces AMS, USA, 2007.

[Zy] A. Zygmund, Trigonometric Series. Cambridge Univ. Press, London and New York, (1959). 


\section{Índice alfabético}

Espacio

$$
\begin{aligned}
& B M O_{r}(\mathbb{D}), 23 \\
& V M O_{r}, 24 \\
& \alpha \text {-growth, } 29 \\
& L^{\infty}(\mathbb{D}), 5 \\
& p \text {-Bergman } q, s, 28 \\
& p \text {-Bergman } q, s \text { pequeño, } 28 \\
& \mathrm{C}(\mathbb{D}), 16 \\
& \text { de Banach, } 1 \\
& \text { de Bergman, } 4 \\
& \text { de Bloch, } 13 \\
& \text { funciones analíticas, } 28 \\
& \text { normado, } 1 \\
& \text { pequeño } \alpha \text {-growth, } 29 \\
& \text { pequeño de Bloch, } 14
\end{aligned}
$$

Fórmula reproductora, 8

Función

de Green, 13

Lacunaria, 43

Núcleo de Bergman, 7

Operador lineal

acotado, 1

Proyección de Bergman, 7

Pseudo-distancia no isotrópica, 49

\section{Teorema}

de Schur, 2 estimación integral, 3 Transformación de Möbius, 11

Transformada de Berezin, 16 
ESPACIOS DE BERGMAN PESADOS

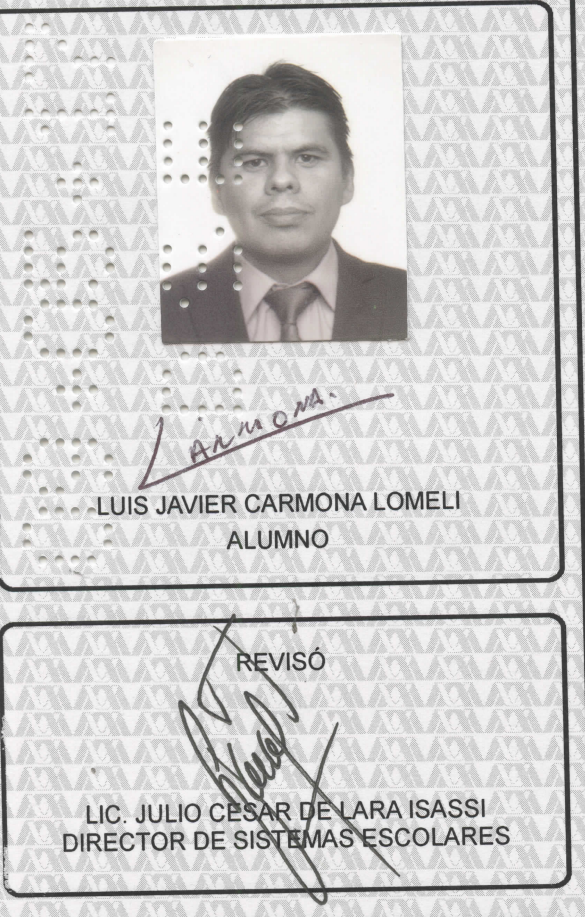

DIRECTOR DE LA DIVISIÓN DE CBI

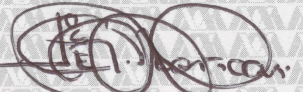

DR. JOSE GILBERTO CORDOBA HERRERA

En la Ciudad de México, se presentaron a las 12:00 horas del día 28 del mes de abril del año 2017 en la Unidad Iztapalapa de la Universidad Autónoma Metropolitana, los suscritos miembros del jurado:

\author{
DR. ANTONI ADAM WAWRZYÑCZYK WILKIEWICZ \\ DRA. MARIA DE LOURDES PALACIOS FABILA \\ DR. LUIS MANUEL TOVAR SANCHEZ \\ DRA. MARIBEL LOAIZA LEYVA \\ DR. LUIS VERDE STAR
}

Bajo la Presidencia del primero y con carácter de Secretario el último, se reunieron a la presentación de la Disertación pública cuya denominación aparece al margen, para la obtención del grado de:

DOCTOR EN CIENCIAS (MATEMATICAS)

DE: LUIS JAVIER CARMONA LOMELI

y de acuerdo con el artículo 78 fracción IV del Reglamento de Estudios Superiores de la Universidad Autónoma Metropolitana, los miembros del jurado resolvieron:

\title{
APROBAR
}

Acto continuo, el presidente del jurado comunicó al interesado el resultado de la evaluación $y$, en caso aprobatorio, le fue tomada la protesta.

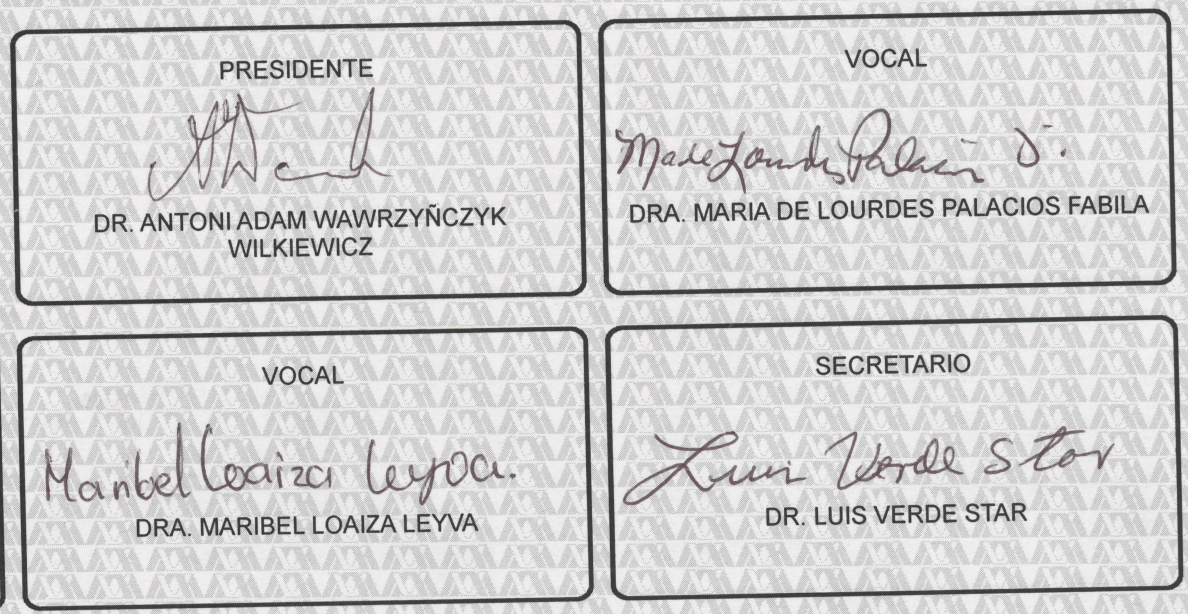

